$\operatorname{Argonne} \underset{\bigotimes}{\bigotimes}$

\title{
April 2008 Monitoring Report for Morrill, Kansas
}

\author{
Environmental Science Division
}

United States Department of Agriculture 


\begin{abstract}
About Argonne National Laboratory
Argonne is a U.S. Department of Energy laboratory managed by UChicago Argonne, LLC under contract DE-AC02-06CH11357. The Laboratory's main facility is outside Chicago, at 9700 South Cass Avenue, Argonne, Illinois 60439. For information about Argonne, see www.anl.gov.
\end{abstract}

\title{
Availability of This Report
}

This report is available, at no cost, at http://www.osti.gov/bridge. It is also available on paper to the U.S. Department of Energy and its contractors, for a processing fee, from:

U.S. Department of Energy

Office of Scientific and Technical Information

P.O. Box 62

Oak Ridge, TN 37831-0062

phone (865) 576-8401

fax (865) 576-5728

reports@adonis.osti.gov

\section{Disclaimer}

This report was prepared as an account of work sponsored by an agency of the United States Government. Reference herein to any specific commercial product, process, or service by trade name, trademark, manufacturer, or otherwise, does not necessarily constitute or imply its endorsement, recommendation, or favoring by the United States Government or any agency thereof. The views and opinions of document authors expressed herein do not necessarily state or reflect those of the United States Government or any agency thereof, Argonne National Laboratory, or UChicago Argonne, LLC. 


\section{April 2008 Monitoring Report for Morrill, Kansas}

by

Applied Geosciences and Environmental Management Section

Environmental Science Division, Argonne National Laboratory

July 2008

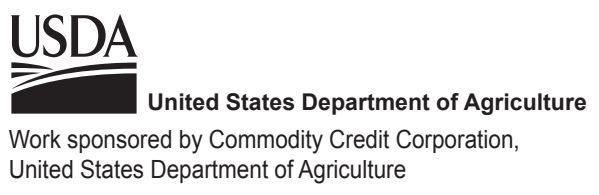




\section{Contents}

Notation

1 Introduction and Background.............................................................................. 1-1

2 Sample Collection and Analysis Activities .................................................................. 2-1

2.1 Measurement of Groundwater Levels .............................................................. 2-1

2.2 Monitoring and Private Well Sampling and Analyses ............................................ 2-. 2-1

2.3 Surface Water and Sediment Sampling and Analyses ........................................... 2-2

2.4 Vegetation Sampling and Analyses .................................................................. 2-3

2.5 Handling and Disposal of Investigation-Derived Waste.......................................... 2-. 2-3

2.6 Quality Control for Sample Collection, Handling, and Analysis.............................. 2-3

3 Results and Discussion.........................................................................................

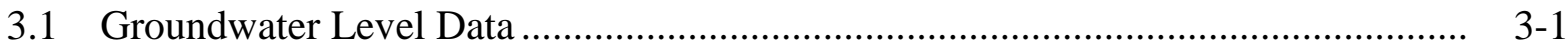

3.2 Groundwater Analysis Results .................................................................... 3-1

3.3 Surface Water and Sediment Analysis Results ....................................................... 3-2

3.4 Vegetation Analysis Results and Observations....................................................... 3-3

4 Conclusions, Issues, and Ongoing Work ………………............................................. 4-1

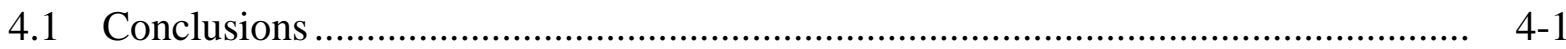

4.2 Issues and Ongoing Work ........................................................................... 4-

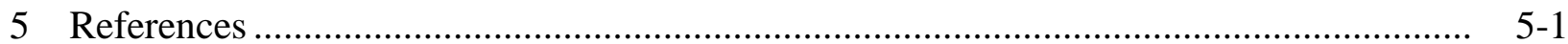

Appendix A: Documentation for the Grimm Irrigation Well ............................................ A-1

Appendix B: Sequence of Sampling Activities at Morrill, Kansas, in April-May 2008 ........ B-1

Appendix C: Analytical Results for Waste Purge Water ……………….............................. C-1

Appendix D: Data Summary for Verification VOCs Analyses by Envirosystems, Inc.......... D-1

\section{Figures}

1.1 Monitoring network at Morrill, as of April 2008

2.1 Locations of surface water and creek bed sediment sampling along Terrapin Creek at Morrill in April 2008 
3.1a Potentiometric surface at Morrill, based on water levels measured manually on January 8, 2007

3.1b Potentiometric surface at Morrill, based on water levels measured manually on July 27,2007

3.1c Potentiometric surface at Morrill, based on water levels measured manually on April 30, 2008

3.2 Hydrographs summarizing results of long-term water level monitoring in wells MW1S-MW4S and MW6S-MW8S at Morrill, from January 1, 2007, to April 30, 2008

3.3 Carbon tetrachloride concentrations in groundwater at Morrill, April 2008

3.4 Lateral extent of the carbon tetrachloride contamination in groundwater at Morrill, as interpreted on the basis of sampling and analysis in April 2008

3.5 Lateral extent of the carbon tetrachloride contamination in groundwater at Morrill, as interpreted on the basis of sampling and analysis in October 2007 (left) and April 2008 (right)

\section{Tables}

3.1 Goundwater levels at Morrill, measured by hand in 2007 and 2008

3.2 Results of analyses at the AGEM Laboratory for volatile organic compounds in groundwater samples collected at Morrill, October 2003

to May 2008

3.3 Field measurements for groundwater samples collected at Morrill, October 2003 to May 2008

3.4 Results of analyses at the AGEM Laboratory for volatile organic compounds in surface water and sediment samples collected at Morrill, March 2007 to April 2008

B.1 Sequence of sampling activities at Morrill in April-May 2008

B-2 


\section{Notation}

AGEM Applied Geosciences and Environmental Management

AMSL above mean sea level

BGL below ground level

${ }^{\circ} \mathrm{C} \quad$ degree(s) Celsius

CCC Commodity Credit Corporation

COC chain of custody

EDB ethylene dibromide

EPA U.S. Environmental Protection Agency

$\mathrm{ft} \quad$ foot (feet)

gpm gallon(s) per minute

hr hour

in. inch(es)

KDHE Kansas Department of Health and Environment

L liter(s)

$\mu \mathrm{g} / \mathrm{kg} \quad$ microgram(s) per kilogram

$\mu \mathrm{g} / \mathrm{L} \quad$ microgram(s) per liter

$\mu \mathrm{S} / \mathrm{cm} \quad$ microsiemen(s) per centimeter

$\mathrm{mg} / \mathrm{L} \quad$ milligram(s) per liter

$\mathrm{mV} \quad$ millivolt(s)

ORP oxidation-reduction potential

SOP standard operating procedure

TOC top of casing

USDA U.S. Department of Agriculture

VOC volatile organic compound 


\section{April 2008 Monitoring Results for Morrill, Kansas}

\section{Introduction and Background}

In September 2005, the Commodity Credit Corporation of the U.S. Department of Agriculture (CCC/USDA) initiated periodic sampling of groundwater in the vicinity of a grain storage facility formerly operated by the CCC/USDA at Morrill, Kansas. The sampling at Morrill is being performed on behalf of the CCC/USDA by Argonne National Laboratory, in accord with a monitoring program approved by the Kansas Department of Health and Environment (KDHE), to monitor levels of carbon tetrachloride contamination identified in the groundwater at this site (Argonne 2004, 2005a). This report provides results for the most recent monitoring event, in April 2008.

Under the KDHE-approved monitoring plan (Argonne 2005b), groundwater was initially sampled twice yearly for a recommended period of two years (in fall 2005, in spring and fall 2006, and in spring and fall 2007). The samples were analyzed for volatile organic compounds (VOCs), as well as for selected geochemical parameters to aid in the evaluation of possible natural contaminant degradation (reductive dechlorination) processes in the subsurface environment. During the recommended two-year period, the originally approved scope of the monitoring was expanded to include vegetation sampling (initiated in October 2006) and surface water and stream bed sediment sampling (initiated in March 2007, after a visual reconnaissance along Terrapin Creek [Argonne 2007a]).

The analytical results for groundwater sampling events at Morrill in September 2005, March 2006, September 2006, March 2007, and October 2007 were documented previously (Argonne 2006a,b, 2007b, 2008). Those results consistently demonstrated the presence of carbon tetrachloride contamination, at levels exceeding the KDHE Tier 2 risk-based screening level $(5.0 \mu \mathrm{g} / \mathrm{L})$ for this compound, in a groundwater plume extending generally south-southeastward from the former CCC/USDA facility, toward Terrapin Creek at the south edge of the town. The results of those five monitoring events gave little indication of consistent changes in the contaminant concentrations at the individual monitoring points or of plume migration. Low levels $(\leq 1.3 \mu \mathrm{g} / \mathrm{L})$ of carbon tetrachloride were persistently detected at monitoring well MW8S, however, on the bank of an intermittent tributary to Terrapin Creek. This observation suggested a possible risk of contamination of the surface waters of the creek. That concern became the driving force for ongoing monitoring and consideration of possible remedial options for Morrill. 
In light of the early findings, in 2006 the CCC/USDA recommended expansion of the approved monitoring program to include the collection and analysis of surface water samples along Terrapin Creek (Argonne 2006a). At the request of the KDHE (2007a), locations for both surface water and shallow sediment sampling were discussed with the KDHE in January 2007. An addendum to the existing monitoring plan and a standard operating procedure (SOP AGEM-15) for sediment sampling were submitted to the KDHE on the basis of these discussions (Argonne 2007c,d). To supplement the original scope of the monitoring, Argonne also sampled natural vegetation along Terrapin Creek in October 2006, April 2007, and July 2007 for analyses for VOCs. The results of the plant tissue analyses were reported previously (Argonne 2008).

The April 2008 sampling event reported here represents a continuation of the two-year monitoring program, as requested by the KDHE (2007b). The sampling is presently conducted, in accord with the monitoring plan (Argonne 2005b) and the addendum to that plan (Argonne 2007a), in a network of 12 monitoring wells and 3 private wells (Figure 1.1), at locations approved by the KDHE.

An event that affects the results of the April 2008 monitoring is the installation of an irrigation well, owned by Kent Grimm, on the south side of Terrapin Creek, near monitoring well MW6S. The WWC-5 well registration form (in Appendix A) indicates that the well diameter is 16 in., that artesian flow is occurring at a rate of $250 \mathrm{gpm}$, and that installation occurred on March 10, 2008. 


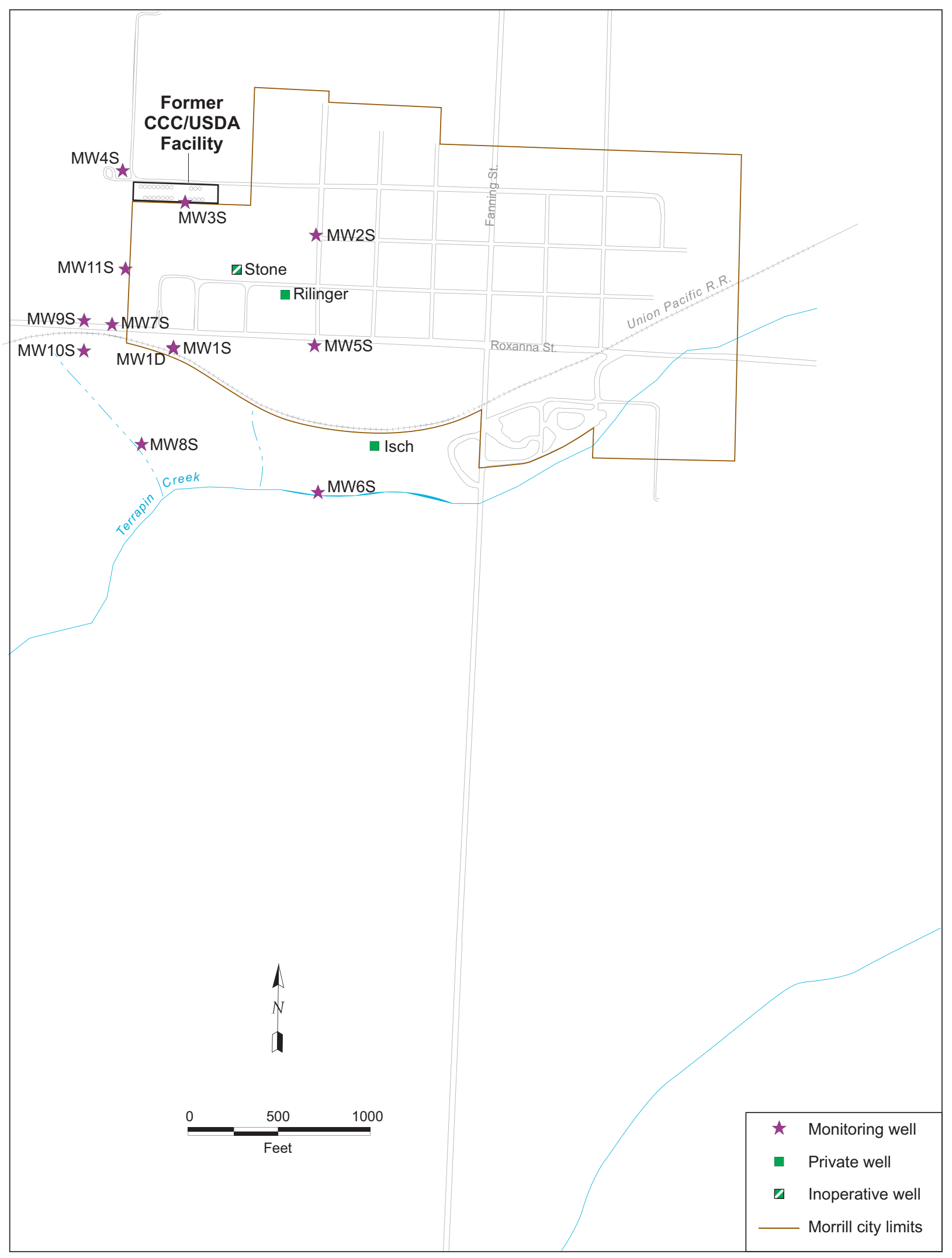

FIGURE 1.1 Monitoring network at Morrill, as of April 2008. 


\section{Sample Collection and Analysis Activities}

\subsection{Measurement of Groundwater Levels}

Before each well was purged in preparation for sampling on April 14-15, 2008, a water level indicator was used (where possible) to measure the depth to groundwater and the total depth from the top of the well casing, with an accuracy of $\pm 0.01 \mathrm{ft}$.

Data recorders currently installed in wells MW1S-MW4S and MW6S-MW8S are gathering long-term data on the groundwater elevation and gradient at Morrill. The data recorders in these wells were downloaded on January 8, 2007; on July 27, 2007; and on April 30, 2008. Water levels were measured manually in all monitoring wells on these dates, as well as in conjunction with the sampling events on October 1-3, 2007, and April 14-15, 2008.

The groundwater level data are discussed in Section 3.1.

\subsection{Monitoring and Private Well Sampling and Analyses}

Monitoring wells MW1D and MW1S-MW11S and the Stone, Isch, and Rilinger private wells (Figure 1.1) were sampled on April 14-15, 2008. Samples were collected in this event by using a low-flow bladder pump.

After measurement of water levels and dissolved oxygen levels, each monitoring point was purged of a small volume. Field measurements of temperature, $\mathrm{pH}$, and conductivity were taken during purging until the measurements stabilized. Field measurements of iron(II) and oxidation reduction potential (ORP) were made as outlined in the monitoring plan (Argonne 2005b). Low-flow sampling was according to U.S. Environmental Protection Agency (EPA) procedure EPA/540/S-95/504 (Puls and Barcelona 1996) and the equipment manufacturers' instructions. All field analyses were performed in accord with procedures in the Master Work Plan (Argonne 2002). The sequence of activities during the April 2008 well sampling event is summarized in Appendix B.

Groundwater samples intended for VOCs and selected geochemical analyses identified in the monitoring plan (Argonne 2005b) were collected in appropriate laboratory containers, 
labeled, packaged, and chilled to $4^{\circ} \mathrm{C}$ by placement in ice-filled coolers. The samples were shipped via an overnight delivery service to the Applied Geosciences and Environmental Management (AGEM) Laboratory at Argonne for VOCs analyses with EPA Method 524.2 (EPA 1995). Separate aliquots of selected samples (chosen in the field) were shipped to Envirosystems, Inc., Columbia, Maryland, for verification VOCs analyses.

As recommended at the end of the original two-year monitoring period (Argonne 2008), routine analyses for the attenuation parameters iron(II), sulfate, sulfide, methane, total organic carbon, carbon dioxide, alkalinity, chloride, and dissolved hydrogen were discontinued after the October 2007 sampling event.

The analytical results are presented and discussed in Section 3.2.

\subsection{Surface Water and Sediment Sampling and Analyses}

At the request of the KDHE (2007a), surface water samples and corresponding samples of the underlying shallow sediments in the creek bed were collected for VOCs analyses in March 2007, October 2007, and April 2008, at five locations along Terrapin Creek (Figure 2.1), as outlined in the addendum (Argonne 2007c) to the monitoring plan (Argonne 2005b). The sampling was conducted in accord with procedures in the Master Work Plan (Argonne 2002) and SOP AGEM-15 (Argonne 2007d). Surface water flow in Terrapin Creek south of Morrill originates at the outfall from an earthen dam and retention pond located approximately 1,900 ft southwest of the former CCC/USDA facility (Figure 2.1). Surface water and sediment sampling location SMB, which is directly downstream of this outfall, is believed to lie upgradient, or cross-gradient, to groundwater flow (and hence possible contaminant migration) from the vicinity of the former CCC/USDA facility. (See Section 3.1.) Sampling locations SM1-SM4 were selected to lie downgradient and downstream from the carbon tetrachloride detections previously identified at MW8S and elsewhere in the monitoring well network.

Samples of surface water were collected in appropriate containers, labeled, preserved at $4^{\circ} \mathrm{C}$, and shipped by an overnight delivery service to the AGEM Laboratory for VOCs analyses with EPA Method 524.2 (EPA 1995). Samples of the shallow creek bed sediments were collected by directly scooping the materials into appropriate laboratory containers (Argonne 
2007d). The samples were labeled, preserved on dry ice, and shipped to the AGEM Laboratory for sample preparation and VOCs analyses with modified EPA Methods 5030B and 8260B.

\subsection{Vegetation Sampling and Analyses}

The April 2008 monitoring event did not include vegetation sampling for the detection of VOCs in mature native vegetation from established trees along Terrapin Creek. The experience gained by the CCC/USDA and Argonne in monitoring phytoremediation treatment programs in Nebraska and Illinois indicates that little uptake of contaminants into tree tissues is expected this early in the growing season (e.g., Argonne 2008b). Tree sampling is scheduled for mid July 2008. Further information is in Section 3.4.

\subsection{Handling and Disposal of Investigation-Derived Waste}

Purge water generated as potentially contaminated investigation-derived waste was containerized on-site. The accumulated purge water was sampled and analyzed by Pace Analytical Services, Inc., Lenexa, Kansas. Methods used were EPA Method 5030/8260 for VOCs and EPA Method 504.1 for ethylene dibromide (EDB). Contamination was not detected. With the approval of KDHE (2008), the waste purge water was discharged on-site. The analytical results for the waste purge water are in Appendix C.

\subsection{Quality Control for Sample Collection, Handling, and Analysis}

The quality control/quality assurance procedures followed during the April 2008 monitoring event are described in detail in the Master Work Plan (Argonne 2002) and SOP AGEM-15 (Argonne 2007b). These procedures are summarized as follows:

- Sample collection and handling activities were monitored by the documentation of samples as they were collected and the use of chain-ofcustody forms and custody seals to ensure sample integrity during handling and shipment. 
- Samples designated for VOCs analyses were received with custody seals intact and at the appropriate preservation temperature. All samples were analyzed within the required holding times.

- Quality control samples (a field blanks and trip blanks) were collected to monitor sample collection and handling activities. Method blanks were analyzed to monitor analytical methodologies. All quality control samples analyzed by the AGEM Laboratory were free of carbon tetrachloride and chloroform contamination.

- Groundwater, surface water, and sediment samples were analyzed for VOCs at the AGEM Laboratory with the purge-and-trap method on a gas chromatograph-mass spectrometer system. Calibration checks with each sample delivery group were required to be within $\pm 20 \%$ of the standard. Surrogate standard determinations performed on samples and blanks were within the specified range of $80-120 \%$ for all samples, in either the initial analysis or a successful reanalysis.

- In accordance with the procedures defined in the Master Work Plan (Argonne 2002), the analyses of water samples at the AGEM Laboratory were verified by a second laboratory. Three groundwater samples collected during the April 14-15, 2008, monitoring event - from monitoring wells MW1S, MW3S, and MW7S - were also submitted to Envirosystems for analysis according to the EPA's Contract Laboratory Program methodology. Lowlevel carbon tetrachloride contamination in monitoring wells MW3S and MW7S $(7.4 \mu \mathrm{g} / \mathrm{L}$ and $8.6 \mu \mathrm{g} / \mathrm{L}$, respectively, in analysis at the AGEM Laboratory) was verified in analysis by Envirosystems $(8.2 \mu \mathrm{g} / \mathrm{L}$ and $10 \mu \mathrm{g} / \mathrm{L}$, respectively). Carbon tetrachloride was not detected in the sample from MW1S by either laboratory. Chloroform was not detected by either laboratory in any of the samples. Methylene chloride was detected in all samples analyzed by Envirosystems, including the associated blanks, but it was not detected in analyses by the AGEM Laboratory. Summary pages for the verification organic analyses for sampling conducted on April 14-15 are in Appendix D. 
- To confirm an apparent effect of the newly installed Grimm irrigation well on contaminant levels observed in the April 14-15, 2008, monitoring event, follow-up sampling was conducted on April 22-23, 2008. In this follow-up event (reported toward the end of Table 3.2), samples were collected from five monitoring wells (MW1S, MW3S, MW5S, MW7S, and MW11S), at one private well monitoring location (Isch), and from the newly installed Grimm irrigation well (TD12) for VOC analysis by both the AGEM Laboratory and Envirosystems. Results for carbon tetrachloride were consistent between the two laboratories, as follows:

No carbon tetrachloride or trace concentrations detected by either laboratory in samples from wells MW1S, MW3S, MW5S, Isch, and TD12.

Low concentrations detected in the sample from well MW7S $(8.3 \mu \mathrm{g} / \mathrm{L}$ in analysis by the AGEM Laboratory; $7.6 \mu \mathrm{g} / \mathrm{L}$ in verification analysis by Envirosystems).

Higher concentrations detected in the sample from MW11S $(42 \mu \mathrm{g} / \mathrm{L}$ by the AGEM Laboratory; $46 \mu \mathrm{g} / \mathrm{L}$ by Envirosystems [verification]).

Summary pages for the verification organic analyses at Envirosystems, Inc. for sampling conducted on April 22-23, 2008, are in Appendix D. 


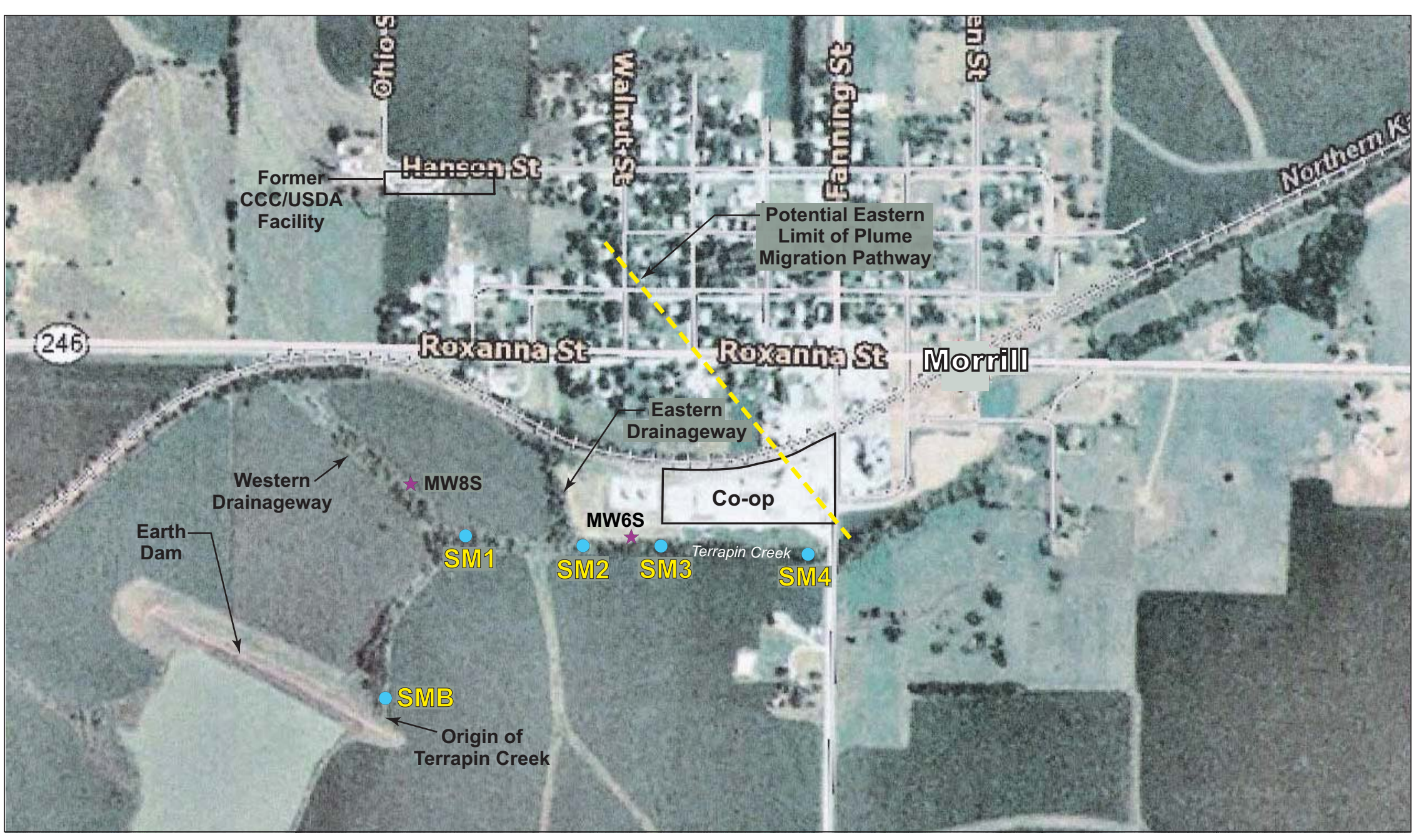

FIGURE 2.1 Locations of surface water and creek bed sediment sampling (SM1-SM4, SMB) along Terrapin Creek at Morrill in April 2008. 


\section{Results and Discussion}

\subsection{Groundwater Level Data}

Depths to groundwater were measured manually in all available monitoring wells on January 8, 2007, July 27, 2007, October 1-3, 2007 (during sampling), April 14-15, 2008 (during sampling), and April 30, 2008. The hand-measured water level data are in Table 3.1. The tables are grouped at the end of the Section 3 text, before the figures.

The potentiometric surface at Morrill, based on manual measurements on January 8, 2007, and July 27, 2007, is depicted in Figures 3.1a,b, respectively. These results indicated a groundwater flow direction toward the south-southeast from the former CCC/USDA facility. Persistently low water levels observed at MW11S empirically suggested the apparent presence of a groundwater "sink" southwest of the former facility in the vicinity of this monitoring well.

The potentiometric surface at Morrill, as interpreted from manual measurements on April 30, 2008, is depicted in Figure 3.1c. The groundwater flow direction on April 30, 2008, appears to be shifted somewhat toward the west, in comparison to the January 8, 2007, and July 27, 2007, depictions (Figures 3.1a,b, respectively). This shift appears to be a response to an effect in the area of MW7S. Additional data from ongoing monitoring is necessary to clarify this observation.

Data from the long-term recording transducers installed in monitoring wells MW1SMW4S and MW6S-MW8S for the period January 2007 to April 2008 are summarized in Figure 3.2. Groundwater levels at the site rose markedly in April-May 2007 and then returned to more typical levels. Responses to individual recharge events are most prominent in wells MW1S-MW3S. The traces for wells MW6S and MW8S show the least variation over time, in keeping with the shallow groundwater at these locations near Terrapin Creek.

\subsection{Groundwater Analysis Results}

The analytical data for VOCs in the groundwater samples collected in April 2008 are in Table 3.2, together with data for the previous sampling events conducted under the KDHE- 
approved monitoring plan (Argonne 2005b). The April 2008 data for carbon tetrachloride in groundwater are illustrated in Figure 3.3.

Carbon tetrachloride was detected at 9 of the 15 monitoring locations, at concentrations ranging from $<1 \mu \mathrm{g} / \mathrm{L}$ (in monitoring wells MW1S and MW9S and in the Isch and Stone private wells) to a maximum of $35 \mu \mathrm{g} / \mathrm{L}$ at MW11S. Low levels of chloroform ( $<1 \mu \mathrm{g} / \mathrm{L}$ to a maximum of $2.7 \mu \mathrm{g} / \mathrm{L}$ at MW1S) were detected in association with carbon tetrachloride at 4 of the monitoring locations.

In comparison to the October 2007 sampling event, the present results (Table 3.2) indicate significant decreases in carbon tetrachloride concentrations at three locations: (1) from $56 \mu \mathrm{g} / \mathrm{L}$ to $<1 \mu \mathrm{g} / \mathrm{L}$ at MW1S; (2) from $61 \mu \mathrm{g} / \mathrm{L}$ to $8.2 \mu \mathrm{g} / \mathrm{L}$ at MW3S; and (3) from $4.0 \mu \mathrm{g} / \mathrm{L}$ to not detected at MW5S. The changes at MW1S, MW3S, and MW5S were confirmed in follow-up sampling (Table 3.2). These changes stand in contrast to the more consistent contaminant concentrations observed in monitoring events in 2003-2007. Figure 3.4 indicates the position of the contaminant plume in April 2008. Figure 3.5 compares the plume configuration in October 2007 (the previous monitoring event) with the configuration in April 2008.

Chloroform concentrations decreased at MW1S, MW3S, and MW5S (as did carbon tetrachloride concentrations), but elsewhere they were consistent with previous results (Table 3.2).

The results of field measurements on the groundwater samples are in Table 3.3.

\subsection{Surface Water and Sediment Analysis Results}

The results of VOCs analyses of the surface water and shallow sediment samples collected (at the request of the KDHE) along Terrapin Creek are in Table 3.4.

No carbon tetrachloride was detected in the surface water samples at an analytical method detection limit of $0.1 \mu \mathrm{g} / \mathrm{L}$. Similarly, no carbon tetrachloride was identified in the associated sediment samples at an analytical method detection limit of $1.0 \mu \mathrm{g} / \mathrm{kg}$. The April 2008 results therefore indicate that the surface waters and underlying sediments of Terrapin Creek have not been impacted by carbon tetrachloride contamination. 


\subsection{Vegetation Analysis Results and Observations}

The results of sampling of natural vegetation on July 26, 2007, at 18 locations along Terrapin Creek and its intermittent tributaries south and southeast of the former CCC/USDA facility were reported previously (Argonne 2008a). The next tree sampling event is scheduled for mid July 2008.

During the winter of 2007-2008, severe ice storms in the Morrill area decimated trees in the town and along the creek. Many trees designated for sampling as part of the continuing baseline study were destroyed. As the 2008 growing season progresses, the health of the remaining baseline trees will be evaluated, as will their reliability for the detection of VOCs. Alternate trees will be selected as replacements for those destroyed or compromised by the severe winter weather, and the baseline sampling set will be reestablished. 
TABLE 3.1 Groundwater levels at Morrill, measured by hand in 2007 and 2008.

\begin{tabular}{|c|c|c|c|c|c|c|c|c|c|c|c|}
\hline \multirow[b]{2}{*}{ Well } & \multirow[b]{2}{*}{$\begin{array}{l}\text { Top of Casing } \\
\text { Elevation } \\
\text { (ft AMSL) }\end{array}$} & \multicolumn{2}{|c|}{ January 8, 2007} & \multicolumn{2}{|c|}{ July 27, 2007} & \multicolumn{2}{|c|}{ October 1-3, 2007} & \multicolumn{2}{|c|}{ April 14-15, 2008} & \multicolumn{2}{|c|}{ April 30, 2008} \\
\hline & & $\begin{array}{l}\text { Depth to } \\
\text { Water } \\
\text { (ft BGL) }\end{array}$ & $\begin{array}{l}\text { Groundwater } \\
\text { Elevation } \\
\text { (ft AMSL) }\end{array}$ & $\begin{array}{l}\text { Depth to } \\
\text { Water } \\
\text { (ft BGL) }\end{array}$ & $\begin{array}{l}\text { Groundwater } \\
\text { Elevation } \\
\text { (ft AMSL) }\end{array}$ & $\begin{array}{l}\text { Depth to } \\
\text { Water } \\
\text { (ft BGL) }\end{array}$ & $\begin{array}{l}\text { Groundwater } \\
\text { Elevation } \\
\text { (ft AMSL) }\end{array}$ & $\begin{array}{l}\text { Depth to } \\
\text { Water } \\
\text { (ft BGL) }\end{array}$ & $\begin{array}{l}\text { Groundwater } \\
\text { Elevation } \\
\text { (ft AMSL) }\end{array}$ & $\begin{array}{l}\text { Depth to } \\
\text { Water } \\
\text { (ft BGL) }\end{array}$ & $\begin{array}{c}\text { Groundwater } \\
\text { Elevation } \\
\text { (ft AMSL) }\end{array}$ \\
\hline MW1S & 1124.68 & 26.79 & 1097.89 & 22.20 & 1102.48 & 21.65 & 1103.03 & 16.20 & 1108.48 & 15.96 & 1108.72 \\
\hline MW1D & 1124.63 & 26.43 & 1098.20 & 22.17 & 1102.46 & 22.75 & 1101.88 & 29.51 & 1095.12 & - & - \\
\hline MW2S & 1137.07 & 37.83 & 1099.24 & 30.50 & 1106.57 & 31.17 & 1105.90 & 23.55 & 1113.52 & 22.84 & 1114.23 \\
\hline MW3S & 1135.76 & 31.73 & 1104.03 & 22.31 & 1113.45 & 22.70 & 1113.06 & 16.95 & 1118.81 & 15.34 & 1120.42 \\
\hline MW4S & 1143.61 & 42.72 & 1100.89 & 30.18 & 1113.43 & 31.11 & 1112.50 & 26.32 & 1117.29 & 24.71 & 1118.90 \\
\hline MW5S & 1122.21 & 26.50 & 1095.71 & 18.47 & 1103.74 & 19.55 & 1102.66 & 11.20 & 1111.01 & 10.75 & 1111.46 \\
\hline MW6S & 1090.97 & 4.93 & 1086.04 & 4.77 & 1086.20 & 5.00 & 1085.97 & 5.15 & 1085.82 & 4.89 & 1086.08 \\
\hline MW7S & 1119.86 & 20.40 & 1099.46 & 13.28 & 1106.58 & 12.40 & 1107.46 & 7.72 & 1112.14 & 7.32 & 1112.54 \\
\hline MW8S & 1098.53 & 3.31 & 1095.22 & 3.06 & 1095.47 & 2.20 & 1096.33 & 0.70 & 1097.83 & 0.61 & 1097.92 \\
\hline MW9S & 1118.31 & 19.29 & 1099.02 & 13.35 & 1104.96 & 14.00 & 1104.31 & 16.58 & 1101.73 & 16.76 & 1101.55 \\
\hline MW10S & 1110.78 & 11.62 & 1099.16 & 6.32 & 1104.46 & 6.95 & 1103.83 & 9.82 & 1100.96 & 9.83 & 1100.95 \\
\hline MW11S & 1133.08 & 36.45 & 1096.63 & 30.90 & 1102.18 & 31.55 & 1101.53 & 29.90 & 1103.18 & 29.81 & 1103.27 \\
\hline
\end{tabular}


TABLE 3.2 Results of analyses at the AGEM Laboratory for volatile organic compounds in groundwater samples collected at Morrill, October 2003 to May 2008.

\begin{tabular}{|c|c|c|c|c|c|}
\hline \multirow[b]{2}{*}{ Location } & \multirow{2}{*}{$\begin{array}{l}\text { Screen } \\
\text { Interval } \\
\text { (ft BGL) }\end{array}$} & \multirow[b]{2}{*}{$\begin{array}{l}\text { Sample } \\
\text { Date }\end{array}$} & \multicolumn{3}{|c|}{ Concentration ( $\mu \mathrm{g} / \mathrm{L})$} \\
\hline & & & $\begin{array}{c}\text { Carbon } \\
\text { Tetrachloride }\end{array}$ & Chloroform & $\begin{array}{l}\text { Methylene } \\
\text { Chloride }\end{array}$ \\
\hline \multirow[t]{8}{*}{ MW1S } & $11-51$ & $10 / 23 / 03$ & 33 & 1.6 & $N D^{a}$ \\
\hline & & $6 / 2 / 04$ & 19 & $0.9 \mathrm{~J}^{\mathrm{b}}$ & ND \\
\hline & & $9 / 13 / 05$ & 35 & 1.7 & ND \\
\hline & & $3 / 22 / 06$ & 40 & 1.8 & ND \\
\hline & & $9 / 20 / 06$ & 23 & $0.9 \mathrm{~J}$ & ND \\
\hline & & $3 / 21 / 07$ & 23 & 1.1 & ND \\
\hline & & $10 / 1 / 07$ & 56 & 2.7 & ND \\
\hline & & $4 / 14 / 08$ & $0.3 \mathrm{~J}$ & ND & ND \\
\hline \multirow[t]{8}{*}{ MW1D } & $63-88$ & $10 / 22 / 03$ & ND & ND & ND \\
\hline & & $6 / 2 / 04$ & ND & ND & ND \\
\hline & & $9 / 13 / 05$ & ND & ND & ND \\
\hline & & $3 / 19 / 06$ & ND & ND & $0.4 \mathrm{~J} \mathrm{~B}^{\mathrm{C}}$ \\
\hline & & $9 / 20 / 06$ & ND & ND & ND \\
\hline & & $3 / 21 / 07$ & ND & ND & ND \\
\hline & & $10 / 1 / 07$ & ND & ND & ND \\
\hline & & $4 / 14 / 08$ & ND & ND & ND \\
\hline \multirow[t]{8}{*}{ MW2S } & $13-53$ & $10 / 22 / 03$ & ND & ND & ND \\
\hline & & $6 / 2 / 04$ & ND & ND & ND \\
\hline & & $9 / 14 / 05$ & ND & ND & ND \\
\hline & & $3 / 21 / 06$ & ND & ND & ND \\
\hline & & $9 / 18 / 06$ & ND & ND & ND \\
\hline & & $3 / 22 / 07$ & ND & ND & ND \\
\hline & & $10 / 3 / 07$ & ND & ND & ND \\
\hline & & 4/15/08 & ND & ND & ND \\
\hline \multirow[t]{8}{*}{ MW3S } & $18-48$ & $10 / 23 / 03$ & 89 & 2.7 & ND \\
\hline & & $6 / 2 / 04$ & 110 & 3.2 & ND \\
\hline & & $9 / 13 / 05$ & 101 & 3.2 & ND \\
\hline & & 3/23/06 & 91 & 2.6 & ND \\
\hline & & $9 / 20 / 06$ & 49 & 1.5 & ND \\
\hline & & $3 / 22 / 07$ & 84 & 2.3 & ND \\
\hline & & 10/3/07 & 61 & 2.0 & ND \\
\hline & & $4 / 14 / 08$ & 8.2 & $0.4 \mathrm{~J}$ & ND \\
\hline \multirow[t]{9}{*}{ MW4S } & $17-47$ & $10 / 21 / 03$ & ND & ND & ND \\
\hline & & $6 / 4 / 04$ & ND & ND & ND \\
\hline & & 9/14/05 & ND & ND & ND \\
\hline & & $3 / 21 / 06$ & ND & ND & ND \\
\hline & & 9/18/06 & ND & ND & ND \\
\hline & & $3 / 22 / 07$ & ND & ND & ND \\
\hline & & $10 / 3 / 07$ & $0.5 \mathrm{~J} \mathrm{R}^{\mathrm{d}}$ & ND & ND \\
\hline & & $1 / 11 / 08$ & ND & ND & ND \\
\hline & & $4 / 14 / 08$ & ND & ND & ND \\
\hline
\end{tabular}


TABLE 3.2 (Cont.)

\begin{tabular}{|c|c|c|c|c|c|}
\hline \multirow[b]{2}{*}{ Location } & \multirow[b]{2}{*}{$\begin{array}{l}\text { Screen } \\
\text { Interval } \\
\text { (ft BGL) }\end{array}$} & \multirow[b]{2}{*}{$\begin{array}{l}\text { Sample } \\
\text { Date }\end{array}$} & \multicolumn{3}{|c|}{ Concentration $(\mu \mathrm{g} / \mathrm{L})$} \\
\hline & & & $\begin{array}{c}\text { Carbon } \\
\text { Tetrachloride }\end{array}$ & Chloroform & $\begin{array}{l}\text { Methylene } \\
\text { Chloride }\end{array}$ \\
\hline MW5S & $15-55$ & $\begin{array}{r}10 / 22 / 03 \\
6 / 2 / 04 \\
9 / 13 / 05 \\
3 / 22 / 06 \\
9 / 20 / 06 \\
3 / 22 / 07 \\
10 / 3 / 07 \\
4 / 14 / 08\end{array}$ & $\begin{array}{r}5.8 \\
7.0 \\
6.3 \\
7.3 \\
6.4 \\
6.5 \\
4.0 \\
\mathrm{ND}\end{array}$ & $\begin{array}{l}\text { ND } \\
\text { ND } \\
0.2 \mathrm{~J} \\
0.2 \mathrm{~J} \\
0.3 \mathrm{~J} \\
0.4 \mathrm{~J} \\
0.3 \mathrm{~J} \\
\mathrm{ND}\end{array}$ & $\begin{array}{l}\text { ND } \\
\text { ND } \\
\text { ND } \\
\text { ND } \\
\text { ND } \\
\text { ND } \\
\text { ND } \\
\text { ND }\end{array}$ \\
\hline MW6S & $10-25$ & $\begin{array}{r}6 / 3 / 04 \\
9 / 14 / 05 \\
3 / 20 / 06 \\
9 / 18 / 06 \\
3 / 21 / 07 \\
10 / 2 / 07 \\
4 / 15 / 08\end{array}$ & $\begin{array}{l}\text { ND } \\
N D \\
N D \\
N D \\
N D \\
N D \\
N D\end{array}$ & $\begin{array}{l}\text { ND } \\
N D \\
N D \\
N D \\
N D \\
N D \\
N D\end{array}$ & $\begin{array}{l}\text { ND } \\
N D \\
N D \\
N D \\
N D \\
N D \\
N D\end{array}$ \\
\hline MW7S & $20-45$ & $\begin{array}{r}6 / 3 / 04 \\
9 / 12 / 05 \\
3 / 22 / 06 \\
9 / 19 / 06 \\
3 / 20 / 07 \\
10 / 1 / 07 \\
4 / 14 / 08\end{array}$ & $\begin{array}{c}18 \\
43 \\
21 \\
38 \\
16 \\
8.1 \\
10\end{array}$ & $\begin{array}{l}\text { ND } \\
1.1 \\
0.4 \mathrm{~J} \\
0.7 \mathrm{~J} \\
0.4 \mathrm{~J} \\
0.2 \mathrm{~J} \\
0.3 \mathrm{~J}\end{array}$ & $\begin{array}{l}\text { ND } \\
N D \\
N D \\
N D \\
N D \\
N D \\
N D\end{array}$ \\
\hline MW8S & $10-25$ & $\begin{array}{r}6 / 3 / 04 \\
9 / 14 / 05 \\
3 / 20 / 06 \\
9 / 19 / 06 \\
3 / 20 / 07 \\
10 / 2 / 07 \\
4 / 15 / 08\end{array}$ & $\begin{array}{l}\text { ND } \\
0.9 \mathrm{~J} \\
0.6 \mathrm{~J} \\
1.3 \\
0.6 \mathrm{~J} \\
0.8 \mathrm{~J} \\
1.1\end{array}$ & $\begin{array}{l}\text { ND } \\
\text { ND } \\
\text { ND } \\
\text { ND } \\
\text { ND } \\
\text { ND } \\
\text { ND }\end{array}$ & $\begin{array}{l}\text { ND } \\
\text { ND } \\
0.4 \mathrm{~J} B \\
\text { ND } \\
\text { ND } \\
\text { ND } \\
\text { ND }\end{array}$ \\
\hline MW9S & $38.83-53.83$ & $\begin{array}{l}3 / 22 / 06 \\
9 / 19 / 06 \\
3 / 20 / 07 \\
10 / 1 / 07 \\
4 / 14 / 08\end{array}$ & $\begin{array}{l}\text { ND } \\
\text { ND } \\
\text { ND } \\
\text { ND } \\
0.8 \mathrm{~J}\end{array}$ & $\begin{array}{l}\text { ND } \\
N D \\
N D \\
N D \\
N D\end{array}$ & $\begin{array}{l}\text { ND } \\
\text { ND } \\
\text { ND } \\
\text { ND } \\
\text { ND }\end{array}$ \\
\hline MW10S & $30-45$ & $\begin{array}{l}3 / 21 / 06 \\
9 / 18 / 06 \\
3 / 21 / 07 \\
10 / 1 / 07 \\
4 / 14 / 08\end{array}$ & $\begin{array}{l}\text { ND } \\
N D \\
N D \\
N D \\
N D\end{array}$ & $\begin{array}{l}\text { ND } \\
N D \\
N D \\
N D \\
N D\end{array}$ & $\begin{array}{l}\text { ND } \\
\text { ND } \\
\text { ND } \\
\text { ND } \\
\text { ND }\end{array}$ \\
\hline MW11S & $53-68$ & $\begin{array}{l}3 / 22 / 06 \\
9 / 19 / 06 \\
3 / 20 / 07 \\
10 / 1 / 07 \\
4 / 15 / 08\end{array}$ & $\begin{array}{l}39 \\
53 \\
37 \\
54 \\
35\end{array}$ & $\begin{array}{l}0.9 \mathrm{~J} \\
1.0 \\
0.8 \mathrm{~J} \\
1.2 \\
0.8 \mathrm{~J}\end{array}$ & $\begin{array}{l}\text { ND } \\
N D \\
N D \\
N D \\
N D\end{array}$ \\
\hline
\end{tabular}


TABLE 3.2 (Cont.)

\begin{tabular}{|c|c|c|c|c|c|}
\hline \multirow[b]{2}{*}{ Location } & \multirow[b]{2}{*}{$\begin{array}{l}\text { Screen } \\
\text { Interval } \\
\text { (ft BGL) }\end{array}$} & \multirow[b]{2}{*}{$\begin{array}{l}\text { Sample } \\
\text { Date }\end{array}$} & \multicolumn{3}{|c|}{ Concentration $(\mu \mathrm{g} / \mathrm{L})$} \\
\hline & & & $\begin{array}{c}\text { Carbon } \\
\text { Tetrachloride }\end{array}$ & Chloroform & $\begin{array}{c}\text { Methylene } \\
\text { Chloride }\end{array}$ \\
\hline \multirow[t]{7}{*}{ Isch } & - & 2/19/04 & ND & ND & ND \\
\hline & & $9 / 14 / 05$ & ND & ND & ND \\
\hline & & $3 / 23 / 06$ & ND & ND & ND \\
\hline & & $9 / 19 / 06$ & ND & ND & ND \\
\hline & & 3/22/07 & ND & ND & ND \\
\hline & & $10 / 3 / 07$ & ND & ND & ND \\
\hline & & $4 / 15 / 08$ & $0.4 \mathrm{~J}$ & ND & ND \\
\hline \multirow[t]{9}{*}{ Rilinger } & - & $6 / 4 / 04$ & ND & ND & ND \\
\hline & & $9 / 14 / 05$ & 2.6 & $0.1 \mathrm{~J}$ & ND \\
\hline & & $3 / 19 / 06$ & ND & ND & $0.4 \mathrm{~J} \mathrm{~B}$ \\
\hline & & $9 / 19 / 06$ & ND & ND & ND \\
\hline & & 3/29/07 & 1.3 & 1.1 & ND \\
\hline & & $10 / 3 / 07$ & $13^{e}$ & $0.4 \mathrm{~J}$ & ND \\
\hline & & $10 / 8 / 07$ & $0.4 \mathrm{~J}$ & ND & ND \\
\hline & & $1 / 11 / 08$ & 6.2 & $0.5 \mathrm{~J}$ & ND \\
\hline & & $4 / 15 / 08$ & 9.9 & $0.4 \mathrm{~J}$ & ND \\
\hline \multirow[t]{7}{*}{ Stone } & $43^{f}$ & $6 / 4 / 04$ & 10 & ND & ND \\
\hline & & $9 / 14 / 05$ & 2.6 & $0.3 \mathrm{~J}$ & ND \\
\hline & & $3 / 19 / 06$ & 14 & $0.8 \mathrm{~J}$ & $0.4 \mathrm{~J} \mathrm{~B}$ \\
\hline & & $9 / 19 / 06$ & 2.1 & ND & ND \\
\hline & & 3/22/07 & 5.4 & $0.3 \mathrm{~J}$ & ND \\
\hline & & $10 / 3 / 07$ & 2.8 & ND & ND \\
\hline & & 4/15/08 & $0.9 \mathrm{~J}$ & ND & ND \\
\hline
\end{tabular}

Follow-up sampling to the April 2008 monitoring event to confirm the status of the contaminant plume

$\begin{array}{lccccc}\text { MW1S } & 11-51 & 4 / 22 / 08 & 0.2 \mathrm{~J} & \text { ND } & \text { ND } \\ \text { MW3S } & 18-48 & 4 / 22 / 08 & 0.7 \mathrm{~J} & \text { ND } & \text { ND } \\ \text { MW5S } & 15-55 & 4 / 23 / 08 & \text { ND } & \text { ND } & \text { ND } \\ \text { MW7S } & 20-45 & 4 / 23 / 08 & 8.3 & 0.2 \mathrm{~J} & \text { ND } \\ \text { MW11S } & 53-68 & 4 / 22 / 08 & 42 & 0.9 \mathrm{~J} & \text { ND } \\ \text { Isch } & - & 4 / 22 / 08 & \text { ND } & \text { ND } & \text { ND }\end{array}$

Sampling of Grimm irrigation well discharge to Terrapin Creek

$\begin{array}{llllll}\text { TD12 } & 27-67 & 4 / 22 / 08 & \text { ND } & \text { ND } & \text { ND }\end{array}$

Sampling of MW1S with the bladder pump intake positioned at 22, 27, and $48 \mathrm{ft} B G L$

$\begin{array}{lccccc}\text { MW1S } & 11-51 & 5 / 1 / 08 & \text { ND } & \text { ND } & \text { ND } \\ & & 5 / 1 / 08 & \text { ND } & \text { ND } & \text { ND } \\ & 5 / 1 / 08 & 0.3 \mathrm{~J} & \text { ND } & \text { ND }\end{array}$

Sampling of MW3S with the bladder pump intake positioned at 26, 38, and $45 \mathrm{ft} B G L$

$\begin{array}{llllll}\text { MW3S } & 18-48 & 5 / 1 / 08 & 0.4 \mathrm{~J} & \text { ND } & \text { ND } \\ & 5 / 1 / 08 & 0.4 \mathrm{~J} & \text { ND } & \text { ND } \\ & & 5 / 1 / 08 & 0.5 \mathrm{~J} & \text { ND } & \text { ND }\end{array}$


TABLE 3.2 (Cont.)

\begin{tabular}{llcccc}
\hline & & & \multicolumn{2}{c}{ Concentration $(\mu \mathrm{g} / \mathrm{L})$} \\
\cline { 3 - 5 } Location & $\begin{array}{c}\text { Screen } \\
\text { Interval } \\
\text { (ft BGL) }\end{array}$ & $\begin{array}{c}\text { Sample } \\
\text { Date }\end{array}$ & $\begin{array}{c}\text { Carbon } \\
\text { Tetrachloride }\end{array}$ & Chloroform & $\begin{array}{c}\text { Methylene } \\
\text { Chloride }\end{array}$ \\
\hline
\end{tabular}

Sampling of MW5S with the bladder pump intake positioned at 20, 28, and $52 \mathrm{ft} B G L$

MW5S

$15-55$

$\begin{array}{ll}5 / 1 / 08 & \text { ND } \\ 5 / 1 / 08 & \text { ND } \\ 5 / 1 / 08 & \text { ND }\end{array}$

ND

ND

$5 / 1 / 08$

ND

ND

ND

$\mathrm{ND}$

a ND, not detected at instrument detection limit of $0.1 \mu \mathrm{g} / \mathrm{L}$.

b Qualifier $\mathrm{J}$ indicates an estimated concentration below the method quantitation limit of $1.0 \mu \mathrm{g} / \mathrm{L}$.

c Qualifier B indicates that the contaminant was present in the associated method blank.

d Qualifier R indicates that the contaminant was present in the associated equipment rinsate. Resampling confirmed that the well was free of contamination.

e Sample collected after recent reactivation of well. Well resampled on 1/8/07 and 1/11/08.

f Total depth. 
TABLE 3.3 Field measurements for groundwater samples collected at Morrill, October 2003 to May 2008.

\begin{tabular}{|c|c|c|c|c|c|c|c|c|c|}
\hline Location & $\begin{array}{l}\text { Screen } \\
\text { Interval } \\
\text { (ft BGL) }\end{array}$ & $\begin{array}{l}\text { Sample } \\
\text { Date }\end{array}$ & $\begin{array}{c}\text { Temperature } \\
\left({ }^{\circ} \mathrm{C}\right)\end{array}$ & $\mathrm{pH}$ & $\begin{array}{l}\text { Conductivity } \\
(\mu \mathrm{S} / \mathrm{cm})\end{array}$ & $\begin{array}{l}\text { Dissolved } \\
\text { Oxygen } \\
\text { (mg/L) }\end{array}$ & $\begin{array}{l}\text { ORP } \\
(\mathrm{mV})\end{array}$ & $\begin{array}{l}\operatorname{Iron}(\mathrm{II}) \\
\text { (mg/L) }\end{array}$ & $\begin{array}{l}\text { Carbon } \\
\text { Dioxide } \\
\text { (mg/L) }\end{array}$ \\
\hline MW1S & $11-51$ & $\begin{array}{r}10 / 23 / 03 \\
6 / 2 / 04 \\
9 / 13 / 05 \\
3 / 22 / 06 \\
9 / 20 / 06 \\
3 / 21 / 07 \\
10 / 1 / 07 \\
4 / 14 / 08\end{array}$ & $\begin{array}{l}14.6 \\
14.4 \\
15.3 \\
15.5 \\
15.7 \\
16.6 \\
16.0 \\
13.9\end{array}$ & $\begin{array}{l}7.14 \\
7.16 \\
6.95 \\
7.23 \\
7.12 \\
6.48 \\
6.80 \\
7.09\end{array}$ & $\begin{array}{r}933 \\
970 \\
1,174 \\
927 \\
973 \\
960 \\
886 \\
1,237\end{array}$ & $\begin{array}{l}-a \\
- \\
7.17 \\
9.94 \\
7.52 \\
5.45 \\
6.79 \\
6.38\end{array}$ & $\begin{array}{r}13 \\
- \\
200 \\
220 \\
- \\
88 \\
128 \\
118\end{array}$ & $\begin{array}{l}- \\
- \\
0 \\
0.01 \\
0.03 \\
0 \\
0 \\
0.02\end{array}$ & $\begin{array}{r}- \\
- \\
55 \\
40 \\
40 \\
40 \\
30 \\
-\end{array}$ \\
\hline MW1D & $63-88$ & $\begin{array}{r}10 / 22 / 03 \\
6 / 2 / 04 \\
9 / 13 / 05 \\
3 / 19 / 06 \\
9 / 20 / 06 \\
3 / 21 / 07 \\
10 / 1 / 07 \\
4 / 14 / 08\end{array}$ & $\begin{array}{l}14.9 \\
13.9 \\
15.5 \\
12.9 \\
12.5 \\
15.3 \\
16.3 \\
14.6\end{array}$ & $\begin{array}{l}6.87 \\
6.87 \\
6.56 \\
6.95 \\
6.93 \\
6.39 \\
6.60 \\
6.99\end{array}$ & $\begin{array}{l}2,620 \\
2,460 \\
2,470 \\
2,460 \\
2,690 \\
2,540 \\
2,230 \\
2,637\end{array}$ & $\begin{array}{l}- \\
- \\
- \\
5.11 \\
- \\
0.08 \\
6.79 \\
0.50\end{array}$ & $\begin{array}{r}25 \\
- \\
- \\
230 \\
- \\
12 \\
5 \\
32\end{array}$ & $\begin{array}{l}- \\
- \\
- \\
0 \\
- \\
0.39 \\
0.44 \\
0.73\end{array}$ & $\begin{array}{r}- \\
- \\
- \\
- \\
- \\
40 \\
45 \\
-\end{array}$ \\
\hline $.0 \mathrm{MW} 2 \mathrm{~S}$ & $13-53$ & $\begin{array}{r}10 / 22 / 03 \\
6 / 2 / 04 \\
9 / 14 / 05 \\
3 / 21 / 06 \\
9 / 18 / 06 \\
3 / 22 / 07 \\
10 / 3 / 07 \\
4 / 15 / 08\end{array}$ & $\begin{array}{l}16.2 \\
16.9 \\
15.2 \\
13.0 \\
13.6 \\
15.2 \\
16.8 \\
11.7\end{array}$ & $\begin{array}{l}6.86 \\
7.07 \\
6.94 \\
7.07 \\
6.99 \\
6.40 \\
6.97 \\
7.23\end{array}$ & $\begin{array}{l}875 \\
861 \\
801 \\
863 \\
844 \\
790 \\
703 \\
742\end{array}$ & $\begin{array}{l}- \\
- \\
7.85 \\
9.40 \\
6.81 \\
5.82 \\
6.70 \\
3.22\end{array}$ & $\begin{array}{r}20 \\
- \\
142 \\
262 \\
69 \\
69 \\
269 \\
75\end{array}$ & $\begin{array}{l}- \\
- \\
- \\
0.14 \\
0 \\
0 \\
0.01 \\
0\end{array}$ & $\begin{array}{r}- \\
- \\
65 \\
25 \\
80 \\
30 \\
30 \\
-\end{array}$ \\
\hline MW3S & $18-48$ & $\begin{array}{r}10 / 23 / 03 \\
6 / 2 / 04 \\
9 / 13 / 05 \\
3 / 23 / 06 \\
9 / 20 / 06 \\
3 / 22 / 07 \\
10 / 3 / 07 \\
4 / 14 / 08\end{array}$ & $\begin{array}{r}16.8 \\
14.2 \\
14.6 \\
8.9 \\
12.9 \\
15.0 \\
15.3 \\
13.7\end{array}$ & $\begin{array}{l}7.23 \\
7.23 \\
7.13 \\
7.16 \\
7.15 \\
6.44 \\
6.97 \\
7.17\end{array}$ & $\begin{array}{l}655 \\
664 \\
663 \\
662 \\
669 \\
578 \\
594 \\
693\end{array}$ & $\begin{array}{l}- \\
- \\
8.82 \\
6.74 \\
7.64 \\
5.90 \\
0.38 \\
3.52\end{array}$ & $\begin{array}{r}6 \\
- \\
223 \\
269 \\
105 \\
261 \\
282 \\
165\end{array}$ & $\begin{array}{l}- \\
- \\
0 \\
0.08 \\
0 \\
0.17 \\
0 \\
0\end{array}$ & $\begin{array}{r}- \\
- \\
100 \\
25 \\
- \\
30 \\
20 \\
-\end{array}$ \\
\hline MW4S & $17-47$ & $\begin{array}{r}10 / 21 / 03 \\
6 / 4 / 04 \\
9 / 14 / 05 \\
3 / 21 / 06 \\
9 / 18 / 06 \\
3 / 22 / 07 \\
10 / 3 / 07 \\
1 / 11 / 08 \\
4 / 14 / 08\end{array}$ & \begin{tabular}{r|}
\multicolumn{1}{c}{} \\
15.4 \\
15.4 \\
6.7 \\
13.1 \\
14.2 \\
16.4 \\
11.3 \\
13.1
\end{tabular} & $\begin{array}{l}7.17 \\
6.93 \\
7.30 \\
7.25 \\
7.25 \\
6.53 \\
6.95 \\
7.56 \\
7.28\end{array}$ & $\begin{array}{l}758 \\
769 \\
751 \\
729 \\
728 \\
765 \\
715 \\
757 \\
783\end{array}$ & $\begin{array}{l}- \\
- \\
8.00 \\
10.90 \\
8.05 \\
5.91 \\
7.40 \\
- \\
3.80\end{array}$ & $\begin{array}{r}- \\
- \\
174 \\
154 \\
41 \\
78 \\
281 \\
- \\
213\end{array}$ & $\begin{array}{l}- \\
- \\
0 \\
0 \\
0 \\
0.10 \\
0.10 \\
- \\
0\end{array}$ & $\begin{array}{r}- \\
- \\
50 \\
25 \\
50 \\
25 \\
30 \\
- \\
-\end{array}$ \\
\hline MW5S & $15-55$ & $\begin{array}{r}10 / 22 / 03 \\
6 / 2 / 04 \\
9 / 13 / 05 \\
3 / 22 / 06 \\
9 / 20 / 06 \\
3 / 22 / 07 \\
10 / 3 / 07 \\
4 / 14 / 08\end{array}$ & $\begin{array}{l}15.3 \\
14.3 \\
16.0 \\
13.9 \\
13.9 \\
15.5 \\
16.5 \\
14.1\end{array}$ & $\begin{array}{l}7.10 \\
7.21 \\
7.04 \\
7.25 \\
7.19 \\
6.50 \\
7.18 \\
6.90\end{array}$ & $\begin{array}{r}816 \\
817 \\
763 \\
781 \\
787 \\
436 \\
850 \\
1,008\end{array}$ & $\begin{array}{r}- \\
- \\
13.90 \\
4.52 \\
5.82 \\
3.98 \\
1.87 \\
3.73\end{array}$ & $\begin{array}{r}6 \\
- \\
228 \\
234 \\
73 \\
159 \\
268 \\
142\end{array}$ & $\begin{array}{l}- \\
- \\
0 \\
0.06 \\
0 \\
0.08 \\
0.04 \\
0.02\end{array}$ & $\begin{array}{l}- \\
- \\
60 \\
35 \\
35 \\
30 \\
25 \\
-\end{array}$ \\
\hline
\end{tabular}


Version 00, 07/25/08

TABLE 3.3 (Cont.)

\begin{tabular}{|c|c|c|c|c|c|c|c|c|c|}
\hline Location & $\begin{array}{l}\text { Screen } \\
\text { Interval } \\
\text { (ft BGL) }\end{array}$ & $\begin{array}{l}\text { Sample } \\
\text { Date }\end{array}$ & $\begin{array}{l}\text { Temperature } \\
\left({ }^{\circ} \mathrm{C}\right)\end{array}$ & $\mathrm{pH}$ & $\begin{array}{l}\text { Conductivity } \\
(\mu \mathrm{S} / \mathrm{cm})\end{array}$ & $\begin{array}{c}\text { Dissolved } \\
\text { Oxygen } \\
\text { (mg/L) }\end{array}$ & $\begin{array}{l}\text { ORP } \\
(\mathrm{mV})\end{array}$ & $\begin{array}{l}\operatorname{Iron}(\mathrm{II}) \\
\text { (mg/L) }\end{array}$ & $\begin{array}{c}\text { Carbon } \\
\text { Dioxide } \\
\text { (mg/L) }\end{array}$ \\
\hline \multirow[t]{7}{*}{ MW6S } & $10-25$ & $6 / 3 / 04$ & 15.1 & 6.89 & 2,410 & - & - & - & - \\
\hline & & $9 / 14 / 05$ & 14.1 & 7.06 & 2,350 & 0.01 & 54 & 0 & 60 \\
\hline & & $3 / 20 / 06$ & 9.8 & 6.91 & 2,360 & 1.37 & 89 & 0.38 & 60 \\
\hline & & $9 / 18 / 06$ & 12.5 & 6.96 & 2,410 & 0.08 & -29 & 0.35 & 85 \\
\hline & & $3 / 21 / 07$ & 18.0 & 6.34 & 2,450 & 0.12 & 75 & 0.78 & 40 \\
\hline & & $10 / 2 / 07$ & 17.1 & 7.33 & 2,280 & 0.20 & 61 & 0.19 & 35 \\
\hline & & $4 / 15 / 08$ & 8.7 & 6.99 & 2,485 & 0.31 & -76 & 0.41 & - \\
\hline \multirow[t]{7}{*}{ MW7S } & $20-45$ & $6 / 3 / 04$ & 13.8 & 7.19 & 763 & - & - & - & - \\
\hline & & $9 / 12 / 05$ & 15.0 & 7.26 & 760 & 8.35 & 240 & 0 & 50 \\
\hline & & $3 / 22 / 06$ & 15.2 & 7.32 & 740 & 5.52 & 268 & 0.03 & 25 \\
\hline & & 9/19/06 & 13.2 & 7.15 & 764 & 7.37 & 114 & 0 & 25 \\
\hline & & $3 / 20 / 07$ & 14.6 & 6.43 & 750 & 5.31 & 95 & 0 & 30 \\
\hline & & $10 / 1 / 07$ & 15.6 & 6.99 & 725 & 7.76 & 269 & 0.01 & 35 \\
\hline & & $4 / 14 / 08$ & 13.4 & 7.21 & 811 & 2.50 & 276 & 0 & - \\
\hline \multirow[t]{7}{*}{ MW8S } & $10-25$ & $6 / 3 / 04$ & 12.8 & 7.12 & 941 & - & - & - & - \\
\hline & & $9 / 14 / 05$ & 14.1 & 7.30 & 853 & 0.02 & 65 & 0 & 40 \\
\hline & & $3 / 20 / 06$ & 12.5 & 7.04 & 954 & 0.90 & 153 & 0.05 & 30 \\
\hline & & 9/19/06 & 11.8 & 7.09 & 903 & 0.58 & 284 & 0.13 & 50 \\
\hline & & $3 / 20 / 07$ & 11.0 & 6.52 & 1,026 & 0.77 & 76 & 0 & 30 \\
\hline & & $10 / 2 / 07$ & 15.2 & 6.76 & 607 & 2.66 & 209 & 0.02 & 25 \\
\hline & & $4 / 15 / 08$ & 10.2 & 7.27 & 1,067 & 1.58 & 170 & 0 & - \\
\hline \multirow[t]{5}{*}{ MW9S } & $38.83-53.83$ & $3 / 22 / 06$ & 14.6 & 7.17 & 715 & 0.41 & 25 & 0 & 35 \\
\hline & & $9 / 19 / 06$ & 13.0 & 7.08 & 707 & 0.10 & 113 & 0 & 55 \\
\hline & & $3 / 20 / 07$ & 14.2 & 6.39 & 714 & 0.21 & 40 & 0 & 20 \\
\hline & & $10 / 1 / 07$ & 15.5 & 7.05 & 664 & 5.50 & 191 & 0 & 30 \\
\hline & & $4 / 14 / 08$ & 12.6 & 7.33 & 709 & 1.93 & 266 & 0.07 & - \\
\hline \multirow[t]{5}{*}{ MW10S } & $30-45$ & $3 / 21 / 06$ & 6.3 & 7.11 & 701 & 2.10 & 88 & 0.01 & 40 \\
\hline & & $9 / 18 / 06$ & 14.3 & 7.17 & 701 & 0.04 & 24 & 0.08 & 60 \\
\hline & & $3 / 21 / 07$ & 14.5 & 6.51 & 720 & 0.88 & 11 & 0 & 30 \\
\hline & & $10 / 1 / 07$ & 16.3 & 6.97 & 664 & 0.35 & 248 & 0.04 & 35 \\
\hline & & $4 / 14 / 08$ & 16.0 & 7.25 & 723 & 1.25 & 181 & 0 & - \\
\hline \multirow[t]{5}{*}{ MW11S } & $53-68$ & $3 / 22 / 06$ & 14.8 & 7.33 & 762 & 9.40 & 237 & 0.06 & 30 \\
\hline & & 9/19/06 & 13.0 & 7.24 & 764 & 1.42 & 158 & 0.02 & 30 \\
\hline & & $3 / 20 / 07$ & 14.6 & 6.33 & 782 & 3.90 & 76 & 0 & 30 \\
\hline & & $10 / 1 / 07$ & 16.4 & 6.49 & 624 & 6.57 & 241 & 0.04 & 35 \\
\hline & & 4/15/08 & 13.9 & 7.30 & 785 & 6.14 & 152 & 0 & - \\
\hline \multirow[t]{7}{*}{ Isch } & - & 2/19/04 & - & - & - & - & - & - & - \\
\hline & & $9 / 14 / 05$ & 20.4 & 6.73 & 2,300 & - & - & - & - \\
\hline & & $3 / 23 / 06$ & 13.0 & 7.23 & 9,400 & - & - & - & - \\
\hline & & 9/19/06 & - & - & - & - & - & - & - \\
\hline & & $3 / 22 / 07$ & - & - & - & - & - & - & - \\
\hline & & $10 / 3 / 07$ & - & - & - & - & - & - & - \\
\hline & & 4/15/08 & 12.6 & 7.33 & 3,160 & - & - & 0.28 & - \\
\hline
\end{tabular}


Version 00, 07/25/08

TABLE 3.3 (Cont.)

\begin{tabular}{|c|c|c|c|c|c|c|c|c|c|}
\hline Location & $\begin{array}{l}\text { Screen } \\
\text { Interval } \\
\text { (ft BGL) }\end{array}$ & $\begin{array}{l}\text { Sample } \\
\text { Date }\end{array}$ & $\begin{array}{c}\text { Temperature } \\
\left({ }^{\circ} \mathrm{C}\right)\end{array}$ & $\mathrm{pH}$ & $\begin{array}{c}\text { Conductivity } \\
(\mu \mathrm{S} / \mathrm{cm})\end{array}$ & $\begin{array}{c}\text { Dissolved } \\
\text { Oxygen } \\
\text { (mg/L) }\end{array}$ & $\begin{array}{l}\text { ORP } \\
(\mathrm{mV})\end{array}$ & $\begin{array}{l}\text { Iron(II) } \\
\text { (mg/L) }\end{array}$ & $\begin{array}{l}\text { Carbon } \\
\text { Dioxide } \\
\text { (mg/L) }\end{array}$ \\
\hline \multirow[t]{8}{*}{ Rillinger } & - & $6 / 4 / 04$ & 15.9 & 6.99 & 2,450 & - & - & - & - \\
\hline & & $9 / 14 / 05$ & - & - & - & - & - & - & - \\
\hline & & $3 / 19 / 06$ & 11.9 & 7.05 & 2,550 & - & - & - & - \\
\hline & & $9 / 19 / 06$ & - & - & 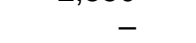 & - & - & - & - \\
\hline & & 3/29/07 & - & - & - & - & - & - & - \\
\hline & & $10 / 3 / 07$ & - & - & - & - & - & - & - \\
\hline & & $1 / 11 / 08$ & 12.2 & 7.46 & 884 & - & - & - & - \\
\hline & & $4 / 15 / 08$ & 12.0 & 7.56 & 868 & - & - & 0 & - \\
\hline \multirow[t]{7}{*}{ Stone } & $43^{b}$ & $6 / 4 / 04$ & 17.1 & 7.35 & 682 & - & - & - & - \\
\hline & & $9 / 14 / 05$ & 17.3 & 6.81 & 638 & - & - & - & - \\
\hline & & $3 / 19 / 06$ & 12.9 & 6.42 & 650 & - & 213 & 0 & - \\
\hline & & $9 / 19 / 06$ & 16.7 & 7.12 & 639 & - & - & - & - \\
\hline & & 3/22/07 & 16.7 & 6.58 & 679 & 4.71 & 19 & 0.28 & 35 \\
\hline & & $10 / 3 / 07$ & 16.1 & 6.97 & 564 & 7.07 & 225 & 0.07 & 25 \\
\hline & & $4 / 15 / 08$ & 11.3 & 7.45 & 557 & - & - & 0 & - \\
\hline
\end{tabular}

Follow-up sampling to the April 2008 monitoring event to confirm the status of the contaminant plume

$\begin{array}{lrrrrrrrrr}\text { MW11S } & 53-68 & 4 / 22 / 08 & 15.1 & 7.25 & 790 & 6.22 & 162 & - & - \\ \text { MW1S } & 11-51 & 4 / 22 / 08 & 15.6 & 6.96 & 1,230 & 6.10 & 133 & - & - \\ \text { MW3S } & 18-48 & 4 / 22 / 08 & 16.0 & 6.99 & 685 & 6.71 & 155 & - & - \\ \text { MW5S } & 15-55 & 4 / 23 / 08 & 14.9 & 6.88 & 1,009 & 3.27 & 184 & - & - \\ \text { MW7S } & 20-45 & 4 / 23 / 08 & 14.0 & 7.00 & 822 & 7.41 & 191 & - & -\end{array}$

Sampling of MW1S with the bladder pump intake positioned at 22, 27, and $48 \mathrm{ft} B G L$

$\begin{array}{lrrrrrrrl}\text { MW1S } & 11-51 & 5 / 1 / 08 & 17.1 & 7.11 & 801 & 3.34 & 104 & - \\ & & 5 / 1 / 08 & 17.0 & 7.10 & 820 & 3.40 & 102 & - \\ & & 5 / 1 / 08 & 16.3 & 7.00 & 1,301 & 3.03 & 118 & -\end{array}$

Sampling of MW3S with the bladder pump intake positioned at 26, 38, and $45 \mathrm{ft} B G L$

MW3

$\begin{array}{lllll}18-48 & 5 / 1 / 08 & 13.2 & 7.17 & 675 \\ & 5 / 1 / 08 & 12.7 & 7.12 & 671 \\ & 5 / 1 / 08 & 12.6 & 7.03 & 675\end{array}$

$5 / 1 / 08 \quad 12.6 \quad 7.03 \quad 675$

75

$\begin{array}{ll}3.83 & 161 \\ 4.21 & 193 \\ 4.57 & 205\end{array}$

Sampling of MW5S with the bladder pump intake positioned at 20, 28, and $52 \mathrm{ft} B G L$

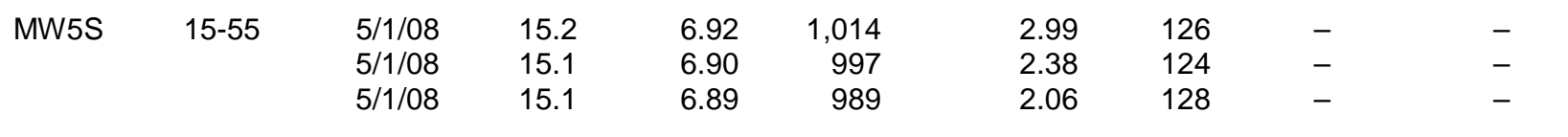

\footnotetext{
a Not measured.

b Total depth.
} 
TABLE 3.4 Results of analyses at the AGEM Laboratory for volatile organic compounds in surface water and sediment samples collected at Morrill, March 2007 to April 2008. ${ }^{a}$

\begin{tabular}{|c|c|c|c|c|c|c|c|}
\hline \multirow[b]{2}{*}{ Location } & \multirow[b]{2}{*}{ Sample } & \multirow[b]{2}{*}{$\begin{array}{l}\text { Sample } \\
\text { Date }\end{array}$} & \multirow[b]{2}{*}{ Medium } & \multicolumn{4}{|c|}{ Concentration ( $\mu \mathrm{g} / \mathrm{L}$ in water; $\mu \mathrm{g} / \mathrm{kg}$ in sediment) } \\
\hline & & & & $\begin{array}{c}\text { Carbon } \\
\text { Tetrachloride }\end{array}$ & Chloroform & $\begin{array}{l}\text { Methylene } \\
\text { Chloride }\end{array}$ & $\begin{array}{l}\text { Quantitation } \\
\text { Limit }\end{array}$ \\
\hline SM1 & MRSM1-W-16572 & 3/22/07 & Water & $N D^{b}$ & ND & ND & 1.0 \\
\hline SM1 & MRSM1-S-16573 & 3/22/07 & Sediment & ND & ND & ND & 10 \\
\hline SM1 & MRSM1-W-16583 & $10 / 8 / 07$ & Water & ND & ND & ND & 1.0 \\
\hline SM1 & MRSM1-S-16584 & $10 / 8 / 07$ & Sediment & ND & ND & ND & 10 \\
\hline SM1 & MRSM1-W-23254 & $4 / 14 / 08$ & Water & ND & ND & ND & 1.0 \\
\hline SM1 & MRSM1-S-23254 & $4 / 14 / 08$ & Sediment & ND & ND & ND & 10 \\
\hline SM2 & MRSM2-W-16574 & 3/22/07 & Water & ND & ND & ND & 1.0 \\
\hline SM2 & MRSM2-S-16575 & 3/22/07 & Sediment & ND & ND & ND & 10 \\
\hline SM2 & MRSM2-W-16585 & $10 / 8 / 07$ & Water & ND & ND & ND & 1.0 \\
\hline SM2 & MRSM2-S-16586 & $10 / 8 / 07$ & Sediment & ND & ND & ND & 10 \\
\hline SM2 & MRSM2-W-23255 & $4 / 14 / 08$ & Water & ND & ND & ND & 1.0 \\
\hline SM2 & MRSM2-S-23255 & $4 / 14 / 08$ & Sediment & ND & ND & ND & 10 \\
\hline SM3 & MRSM3-W-16576 & 3/22/07 & Water & ND & ND & ND & 1.0 \\
\hline SM3 & MRSM3-S-16577 & 3/22/07 & Sediment & ND & ND & ND & 10 \\
\hline SM3 & MRSM3-W-16587 & $10 / 8 / 07$ & Water & ND & ND & ND & 1.0 \\
\hline SM3 & MRSM3-S-16588 & $10 / 8 / 07$ & Sediment & ND & ND & ND & 10 \\
\hline SM3 & MRSM3-W-23256 & $4 / 14 / 08$ & Water & ND & ND & ND & 1.0 \\
\hline SM3 & MRSM3-S-23256 & $4 / 14 / 08$ & Sediment & ND & ND & ND & 10 \\
\hline SM4 & MRSM4-W-16578 & 3/22/07 & Water & ND & ND & ND & 1.0 \\
\hline SM4 & MRSM4-S-16579 & 3/22/07 & Sediment & ND & ND & ND & 10 \\
\hline SM4 & MRSM4-W-16589 & $10 / 8 / 07$ & Water & ND & ND & ND & 1.0 \\
\hline SM4 & MRSM4-S-16590 & $10 / 8 / 07$ & Sediment & ND & ND & ND & 10 \\
\hline SM4 & MRSM4-W-23257 & $4 / 14 / 08$ & Water & ND & ND & ND & 1.0 \\
\hline SM4 & MRSM4-S-23257 & $4 / 14 / 08$ & Sediment & ND & ND & ND & 10 \\
\hline SMB & MRSMB-W-16570 & 3/22/07 & Water & ND & ND & ND & 1.0 \\
\hline SMB & MRSMB-S-16571 & $3 / 22 / 07$ & Sediment & ND & ND & ND & 10 \\
\hline SMB & MRSMB-W-16581 & $10 / 8 / 07$ & Water & ND & ND & ND & 1.0 \\
\hline SMB & MRSMB-S-16582 & $10 / 8 / 07$ & Sediment & ND & ND & ND & 10 \\
\hline SMB & MRSMB-W-23258 & $4 / 14 / 08$ & Water & ND & ND & ND & 1.0 \\
\hline SMB & MRSMB-S-23258 & $4 / 14 / 08$ & Sediment & ND & ND & ND & 10 \\
\hline
\end{tabular}

a Analyses conducted at the AGEM Laboratory, Argonne, Illinois, with EPA Method 524.2 for surface water samples or modified EPA Method 5030B/8260B for sediment samples.

b ND, not detected at the instrument detection limit of $0.1 \mu \mathrm{g} / \mathrm{L}$ for surface water samples or $1.0 \mu \mathrm{g} / \mathrm{kg}$ for sediment samples. 


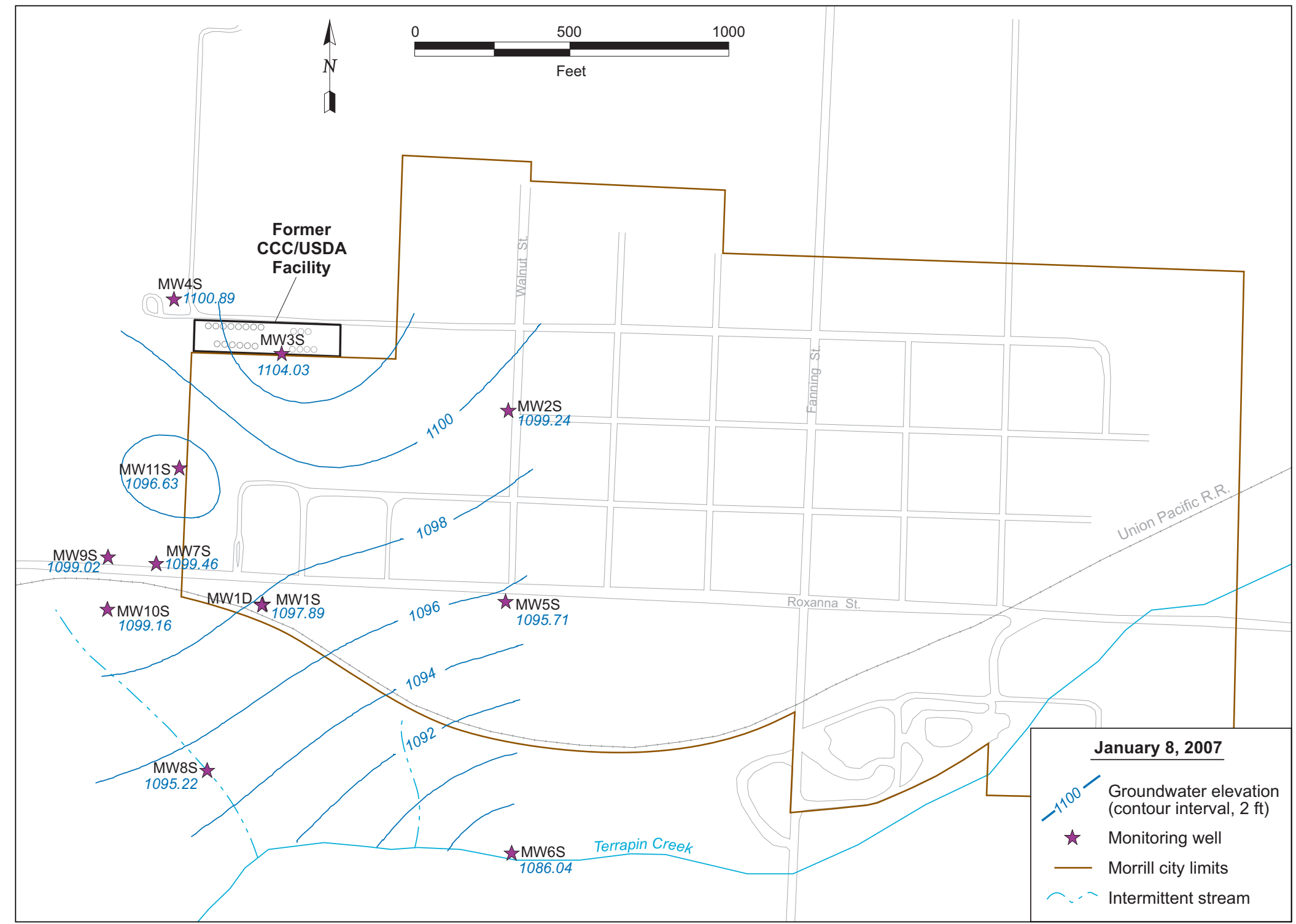

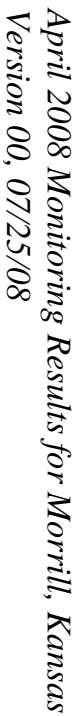

FIGURE 3.1a Potentiometric surface at Morrill, based on water levels measured manually on January 8, 2007. 


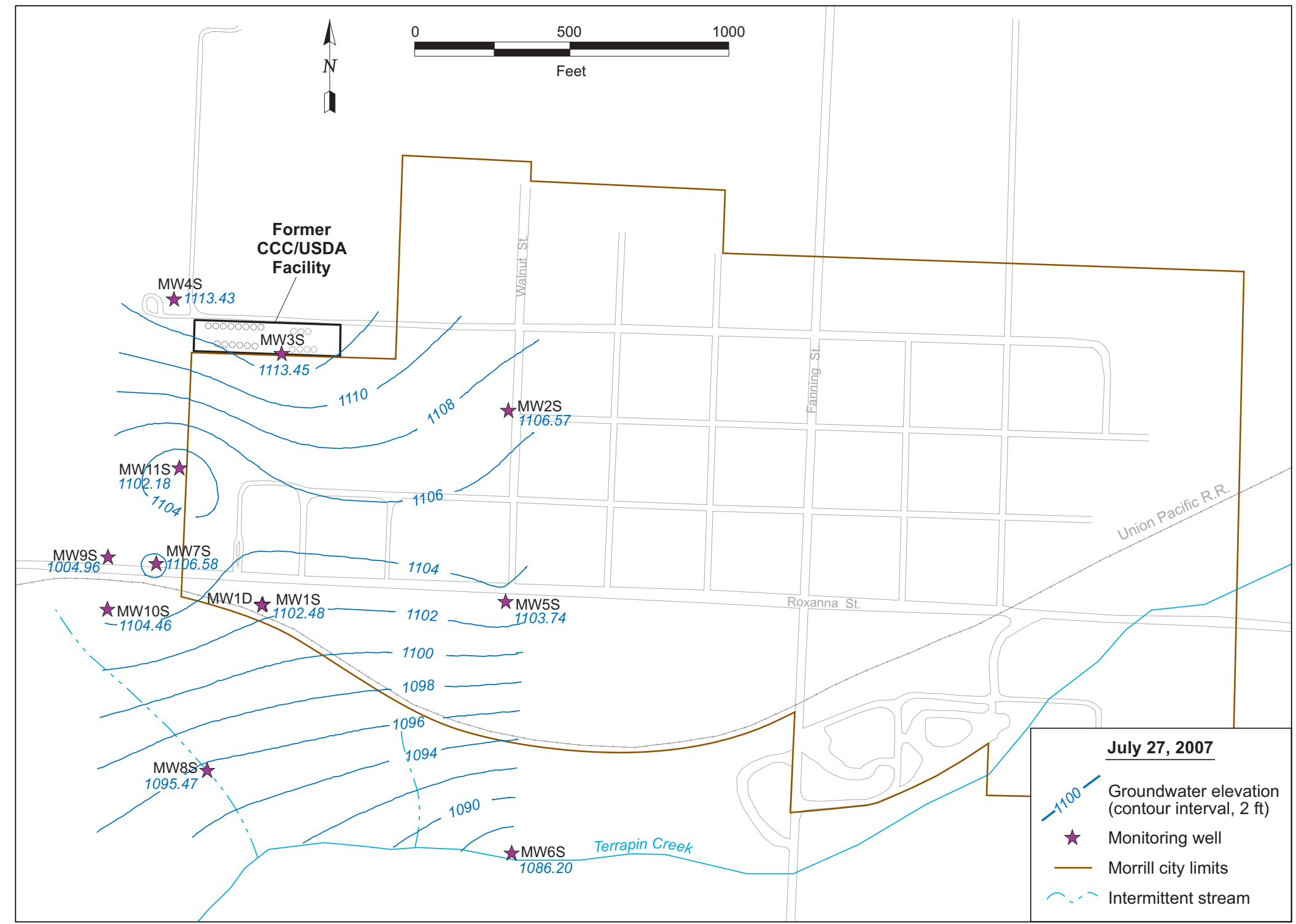

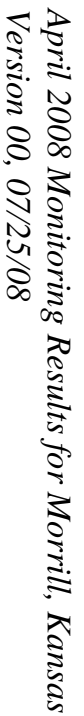

FIGURE 3.1b Potentiometric surface at Morrill, based on water levels measured manually on July 27, 2007. 


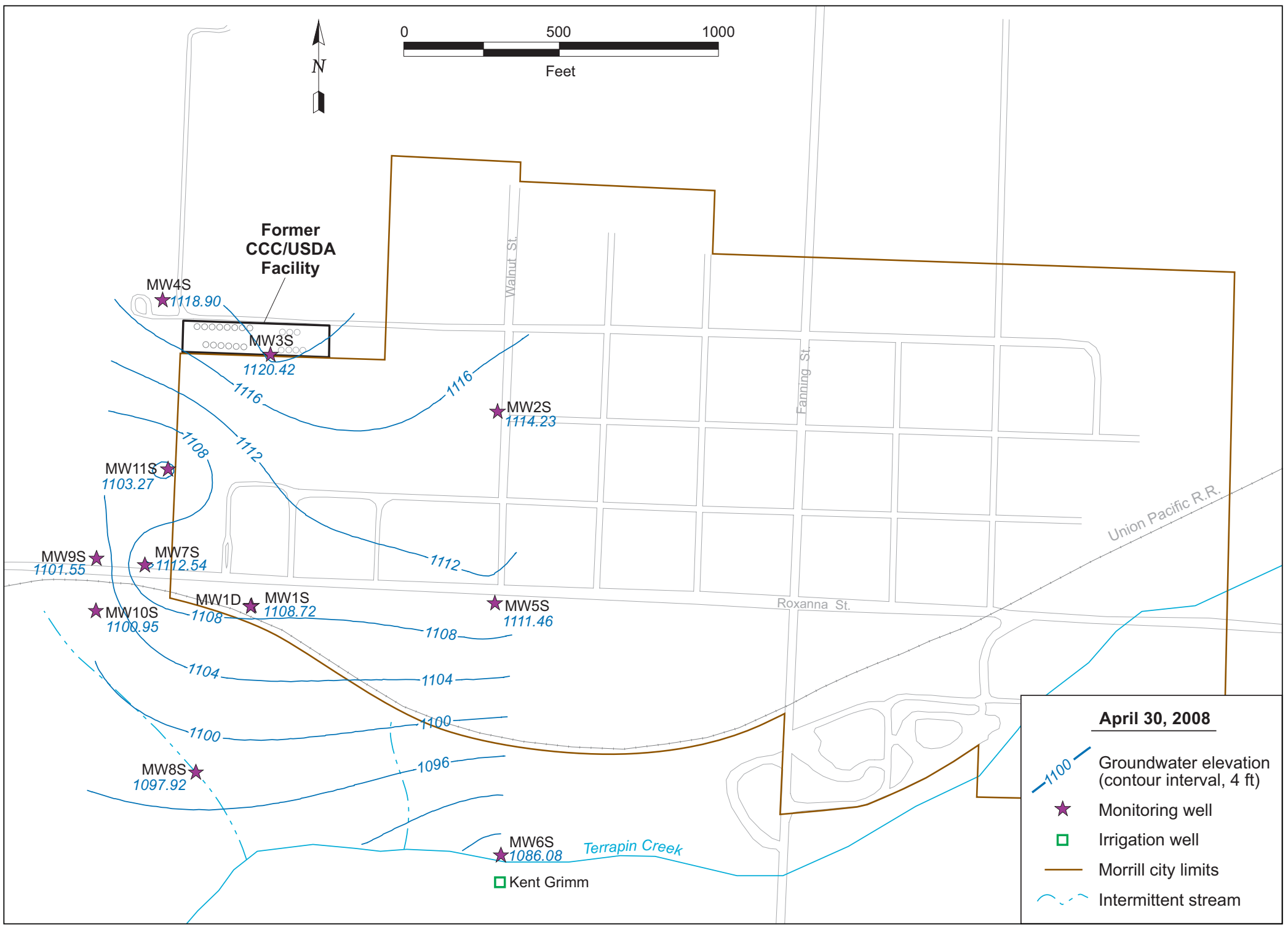

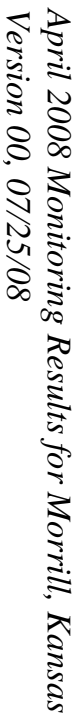

FIGURE 3.1c Potentiometric surface at Morrill, based on water levels measured manually on April 30, 2008. 


\section{Morrill Water Levels}

1-1-07 to 4-30-08

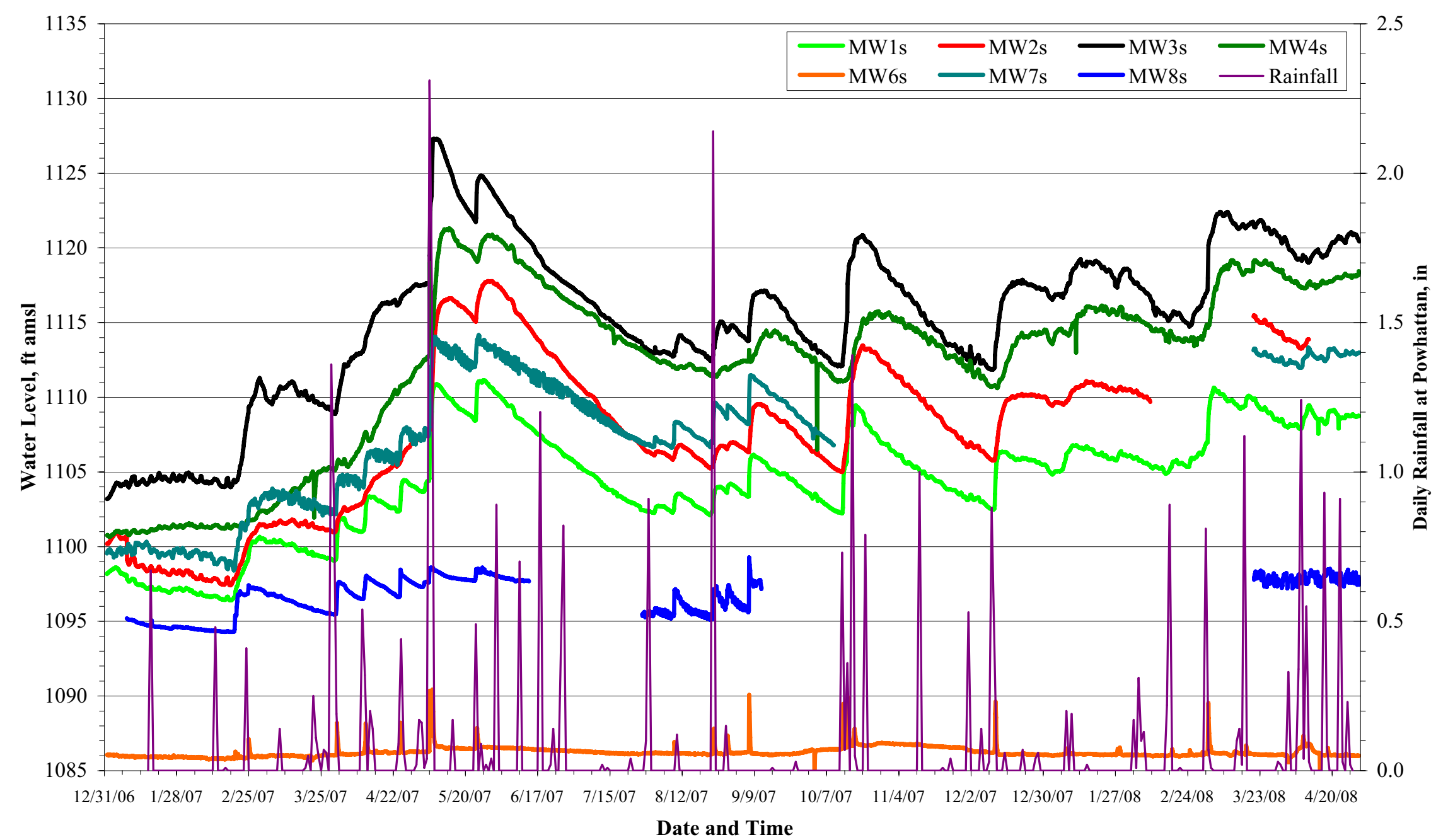

FIGURE 3.2 Hydrographs summarizing results of long-term water level monitoring in wells MW1S-MW4S and MW6S-MW8S at Morrill, from January 1, 2007, to April 30, 2008. 


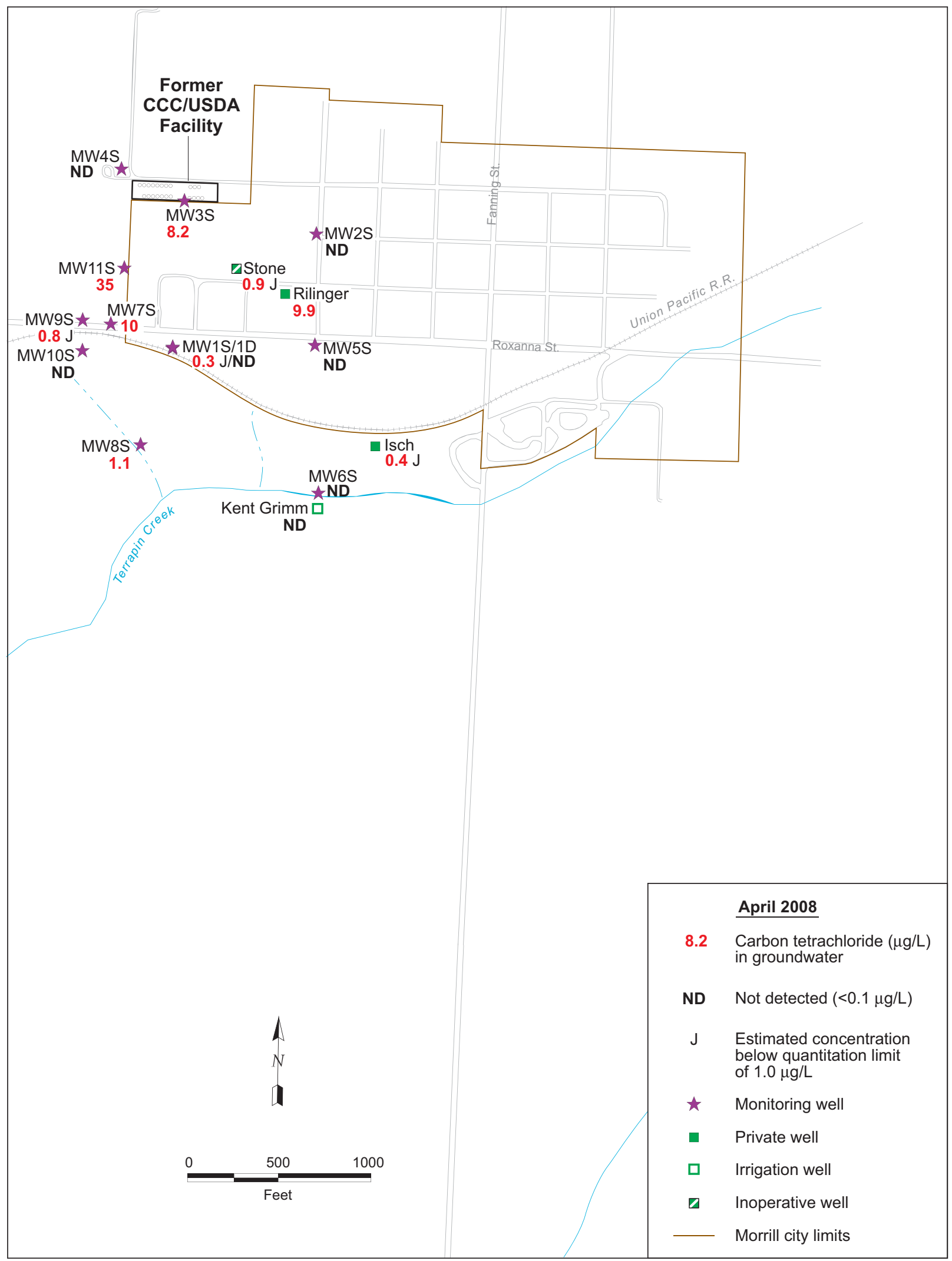

FIGURE 3.3 Carbon tetrachloride concentrations in groundwater at Morrill, April 2008. 


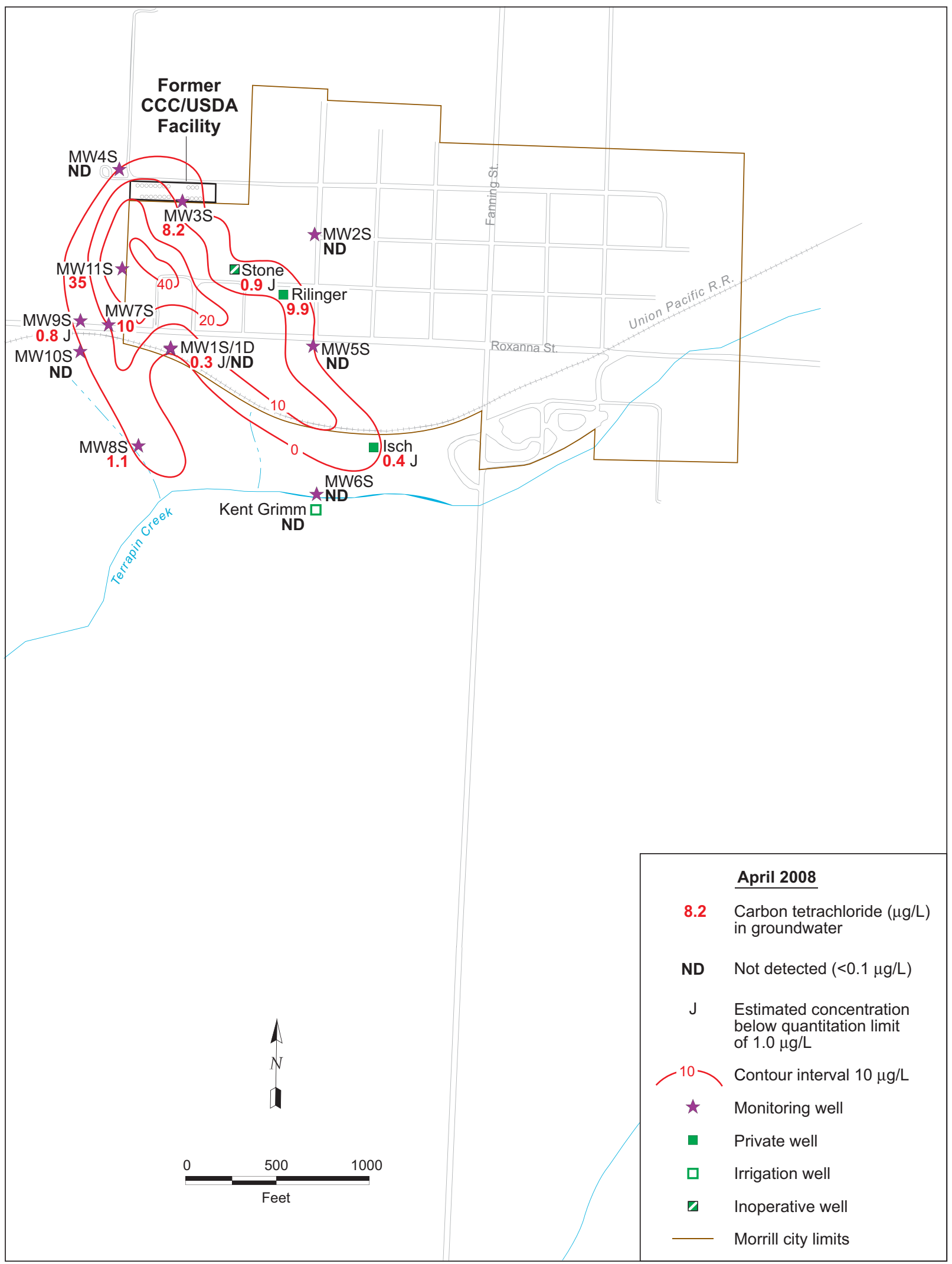

FIGURE 3.4 Lateral extent of the carbon tetrachloride contamination in groundwater at Morrill, as interpreted on the basis of sampling and analysis in April 2008. 

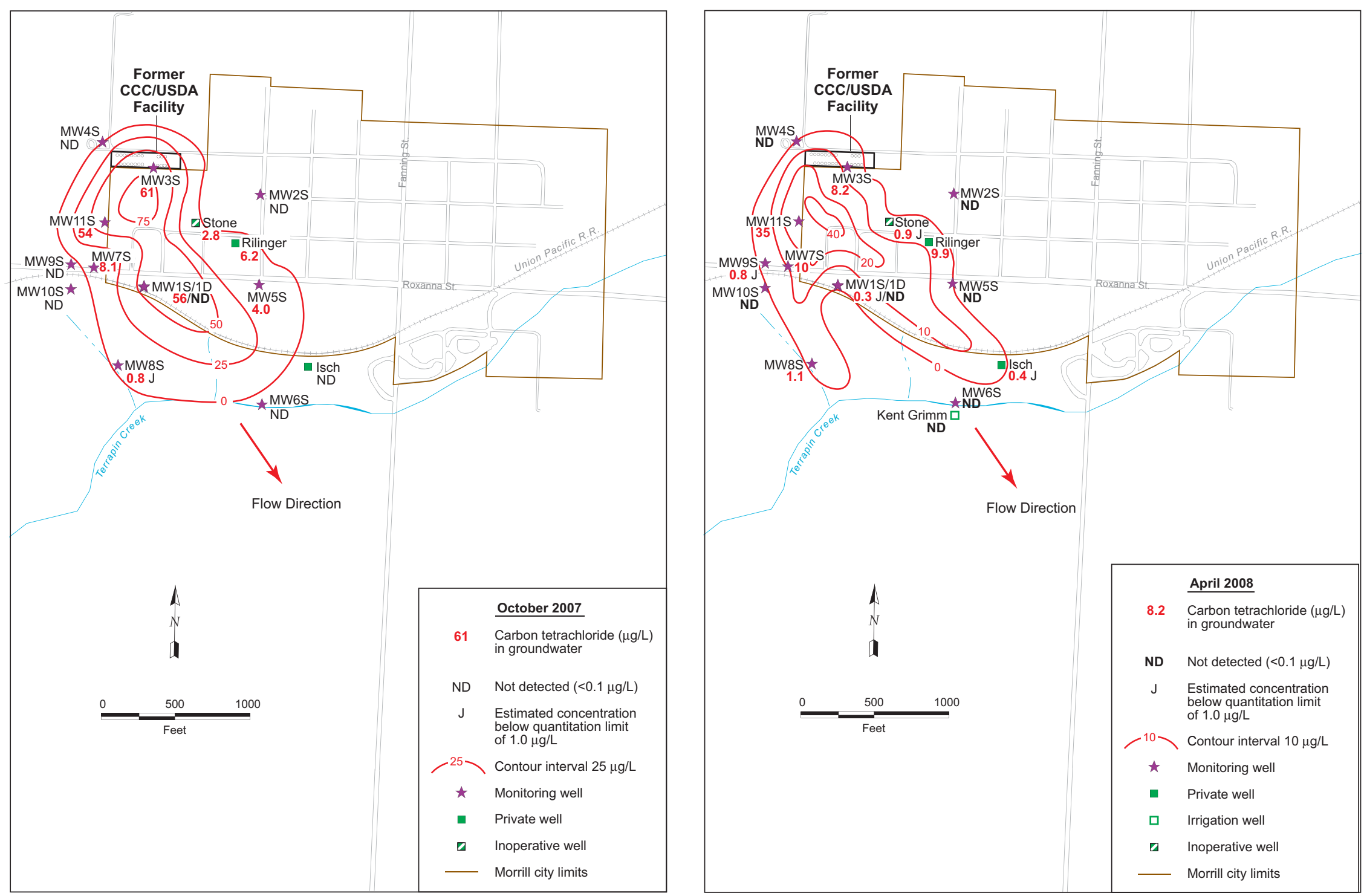

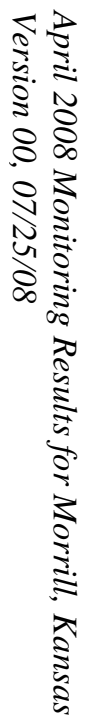

FIGURE 3.5 Lateral extent of the carbon tetrachloride contamination in groundwater at Morrill, as interpreted on the basis of sampling and analysis in October 2007 (left) and April 2008 (right). 


\section{Conclusions, Issues, and Ongoing Work}

\subsection{Conclusions}

The findings of the April 2008 monitoring event at Morrill support the following conclusions:

- Measurements of groundwater levels, made both manually and through the use of automatic recorders, in 2003-2007, consistently indicated an apparent direction of groundwater flow to the south-southeast from the former CCC/USDA facility. Measurements in April 2008 suggested a potential shift in the groundwater flow direction toward the west. Continued monitoring is necessary to clarify this observation.

- In sampling events in 2003-2007, contaminant concentrations had been generally consistent. In the April 2008 sampling event, carbon tetrachloride concentrations at three locations decreased significantly, from $56 \mu \mathrm{g} / \mathrm{L}$ to $<1 \mu \mathrm{g} / \mathrm{L}$ at MW1S, from $61 \mu \mathrm{g} / \mathrm{L}$ to $8.2 \mu \mathrm{g} / \mathrm{L}$ at MW3S, and from $4.0 \mu \mathrm{g} / \mathrm{L}$ to not detected at MW5S.

- No carbon tetrachloride contamination was detected in surface waters or shallow stream bed sediments sampled (at the request of the KDHE) at five locations along Terrapin Creek, downgradient of the former CCC/USDA facility.

\subsection{Issues and Ongoing Work}

The following issues and recommendations merit consideration in planning for ongoing work at Morrill:

- The significant changes in carbon tetrachloride concentrations and the plume configuration observed in the April 2008 sampling were confirmed with repeated sampling. The only known concurrent change in the hydrogeologic system at Morrill was the installation, in March 2008, of a new, high-capacity 
irrigation well on the south side of Terrapin Creek. Further monitoring will be required, and is imperative, in order to fully characterize the well's effects on the hydrogeologic system. Nevertheless, a reasonable preliminary assumption is that the well is influencing the downgradient plume as it moves toward Terrapin Creek.

- Sampling will continue at the frequency previously established under the approved monitoring plan (Argonne 2005b). Sampling events will include groundwater sampling from the existing network of 12 monitoring wells and 3 private wells, as well as sampling of surface waters and stream bed sediments at the 5 established locations along Terrapin Creek.

- Tree sampling is scheduled for mid July 2008. Severe ice storms in the Morrill area during winter 2007-2008 destroyed many trees designated for sampling as part of the continuing baseline study. The health of the remaining baseline trees will be evaluated during the 2008 growing season, as will their reliability for the detection of VOCs. Alternate trees will be selected as necessary to reestablish the baseline sampling set. 


\section{References}

Argonne, 2002, Final Master Work Plan: Environmental Investigations at Former CCC/USDA Facilities in Kansas, 2002 Revision, ANL/ER/TR-02/004, prepared for the Commodity Credit Corporation, U.S. Department of Agriculture, Washington, D.C., by Argonne National Laboratory, Argonne, Illinois, December.

Argonne, 2004, Final Phase I-Phase II Interim Report: Expedited Site Characterization, Morrill, Kansas, ANL/ER/TR-04/001, prepared for the Commodity Credit Corporation, U.S. Department of Agriculture, Washington, D.C., by Argonne National Laboratory, Argonne, Illinois, December.

Argonne, 2005a, Final Report, Monitoring Well Installation and Sampling, 2004, at Morrill, Kansas, ANL/ER/TR-04/010, prepared for the Commodity Credit Corporation, U.S. Department of Agriculture, Washington, D.C., by Argonne National Laboratory, Argonne, Illinois, November.

Argonne, 2005b, Final Work Plan: Groundwater Monitoring at Morrill, Kansas, ANL/ER/TR05/003, prepared for the Commodity Credit Corporation, U.S. Department of Agriculture, Washington, D.C., by Argonne National Laboratory, Argonne, Illinois, August.

Argonne, 2006a, Draft Report: Groundwater Monitoring at Morrill, Kansas, in September 2005 and March 2006, with Expansion of the Monitoring Network in January 2006, ANL/EVS/AGEM/TR-06-09, prepared for the Commodity Credit Corporation, U.S. Department of Agriculture, Washington, D.C., by Argonne National Laboratory, Argonne, Illinois, September.

Argonne, 2006b, September 2006 Monitoring Results for Morrill, Kansas, ANL/EVS/AGEM/CHRON-1016, prepared for the Commodity Credit Corporation, U.S. Department of Agriculture, Washington, D.C., by Argonne National Laboratory, Argonne, Illinois, December.

Argonne, 2007a, Reconnaissance Visit along Terrapin Creek, South of the Former CCC/USDA Grain Bin Site and the Exiting Co-op in Morrill, Kansas, January 2007, ANL/EVS/AGEM/CHRON-1042, prepared for the Commodity Credit Corporation, 
U.S. Department of Agriculture, Washington, D.C., by Argonne National Laboratory, Argonne, Illinois, May 3.

Argonne, 2007b, March-April 2007 Monitoring Results for Morrill, Kansas, ANL/EVS/AGEM/TR-07-06, prepared for the Commodity Credit Corporation, U.S. Department of Agriculture, by Argonne National Laboratory, Argonne, Illinois, May.

Argonne, 2007c, Proposed Addendum to the Groundwater Monitoring Plan for Morrill, Kansas, ANL/EVS/AGEM/CHRON-1042, prepared for the Commodity Credit Corporation, U.S. Department of Agriculture, Washington, D.C., by Argonne National Laboratory, Argonne, Illinois, May 3.

Argonne, 2007d, AGEM-15: Standard Operating Procedure for Sediment Sampling, ANL/EVS/AGEM/CHRON-1042, prepared for the Commodity Credit Corporation, U.S. Department of Agriculture, Washington, D.C., by Argonne National Laboratory, Argonne, Illinois, February 21.

Argonne, 2008a, October 2007 Monitoring Results for Morrill, Kansas, ANL/EVS/AGEM/TR08-02, prepared for the Commodity Credit Corporation, U.S. Department of Agriculture, Washington, D.C., by Argonne National Laboratory, Argonne, Illinois, March.

Argonne, 2008b, Summary of Operations and Performance for the Murdock Site Restoration Project in 2007, ANL/EVS/AGEM/TR-08-07, prepared for the Commodity Credit Corporation, U.S. Department of Agriculture, Washington, D.C., by Argonne National Laboratory, Argonne, Illinois, March.

EPA, 1995, Method 524.2: Measurement of Purgeable Organic Compounds in Water by Capillary Column Gas Chromatography/Mass Spectrometry, Revision 4.1, edited by J.W. Munch, National Exposure Research Laboratory, Office of Research and Development, U.S. Environmental Protection Agency, Cincinnati, Ohio.

KDHE, 2007a, letter from C. Carey (Bureau of Environmental Remediation, Kansas Department of Health and Environment, Topeka, Kansas) to C. Roe (Commodity Credit Corporation, U.S. Department of Agriculture, Washington, D.C.) regarding Draft Report: Groundwater 
Monitoring at Morrill, Kansas, in September 2005 and March 2006, with Expansion of the Monitoring Network in January 2006, January 5.

KDHE, 2007b, letter from C. Carey (Bureau of Environmental Remediation, Kansas Department of Health and Environment, Topeka, Kansas) to C. Roe (Commodity Credit Corporation, U.S. Department of Agriculture, Washington, D.C.) regarding March-April 2007 Monitoring Results for Morrill, Kansas, August 1.

KDHE, 2008, electronic mail message from E. McWilliams (Bureau of Environmental Remediation, Kansas Department of Health and Environment, Topeka, Kansas) to L. Larsen (Larsen and Associates, Inc., Lawrence, Kansas), July 1.

Puls, R.W., and Barcelona, M.J., 1996, "Low-Flow (Minimal Drawdown) Ground-Water Sampling Procedures," EPA/540/S-95/504, in Ground Water Issue, Superfund Technology Support Center for Ground Water, National Risk Management Research Laboratory, Ada, Oklahoma, April (www.epa.gov/tio/tsp/download/lwflw2a.pdf). 


\section{Appendix A:}

\section{Documentation for the Grimm Irrigation Well}




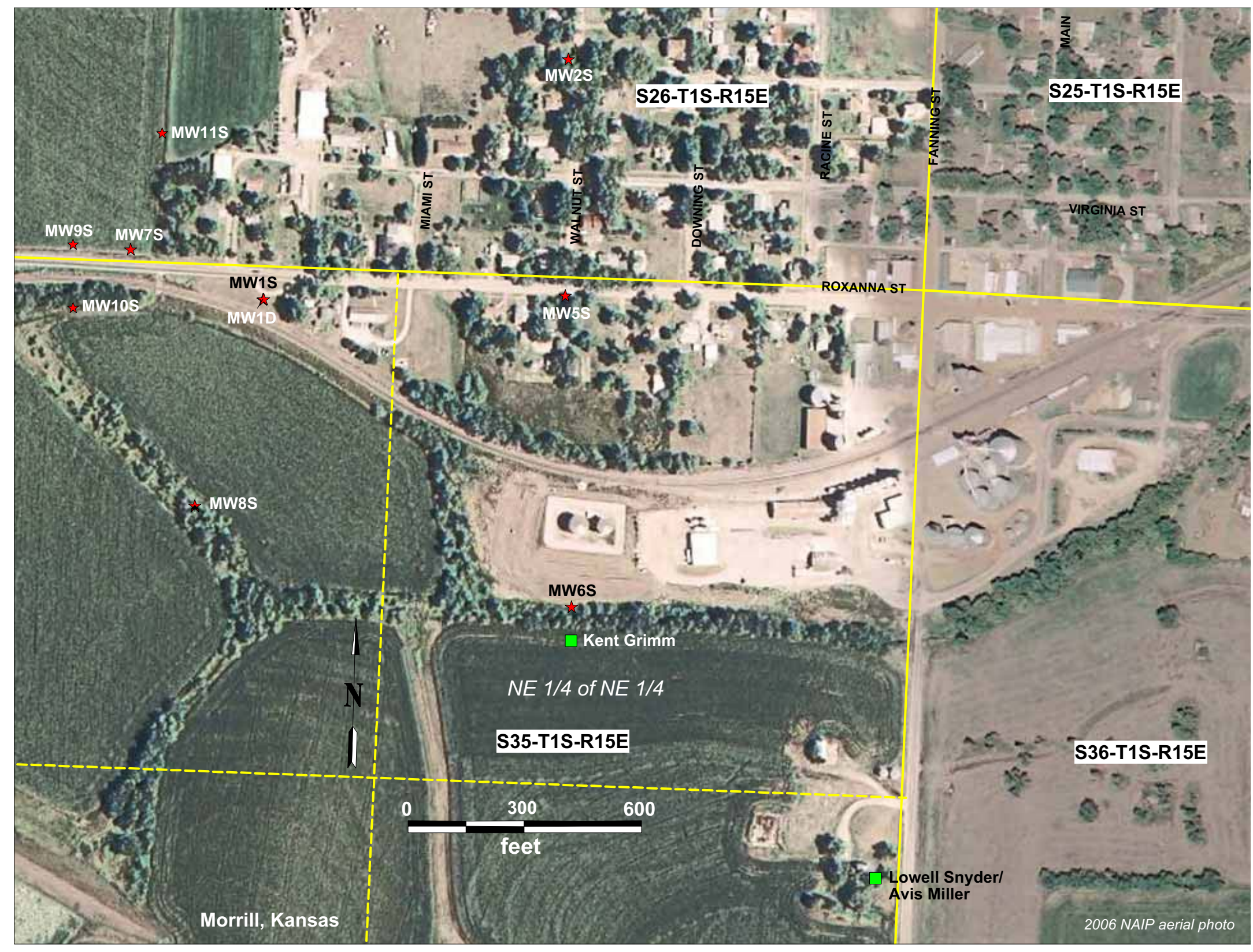

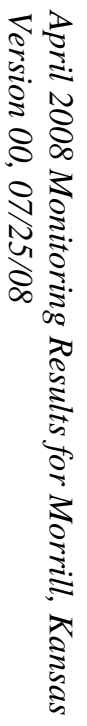




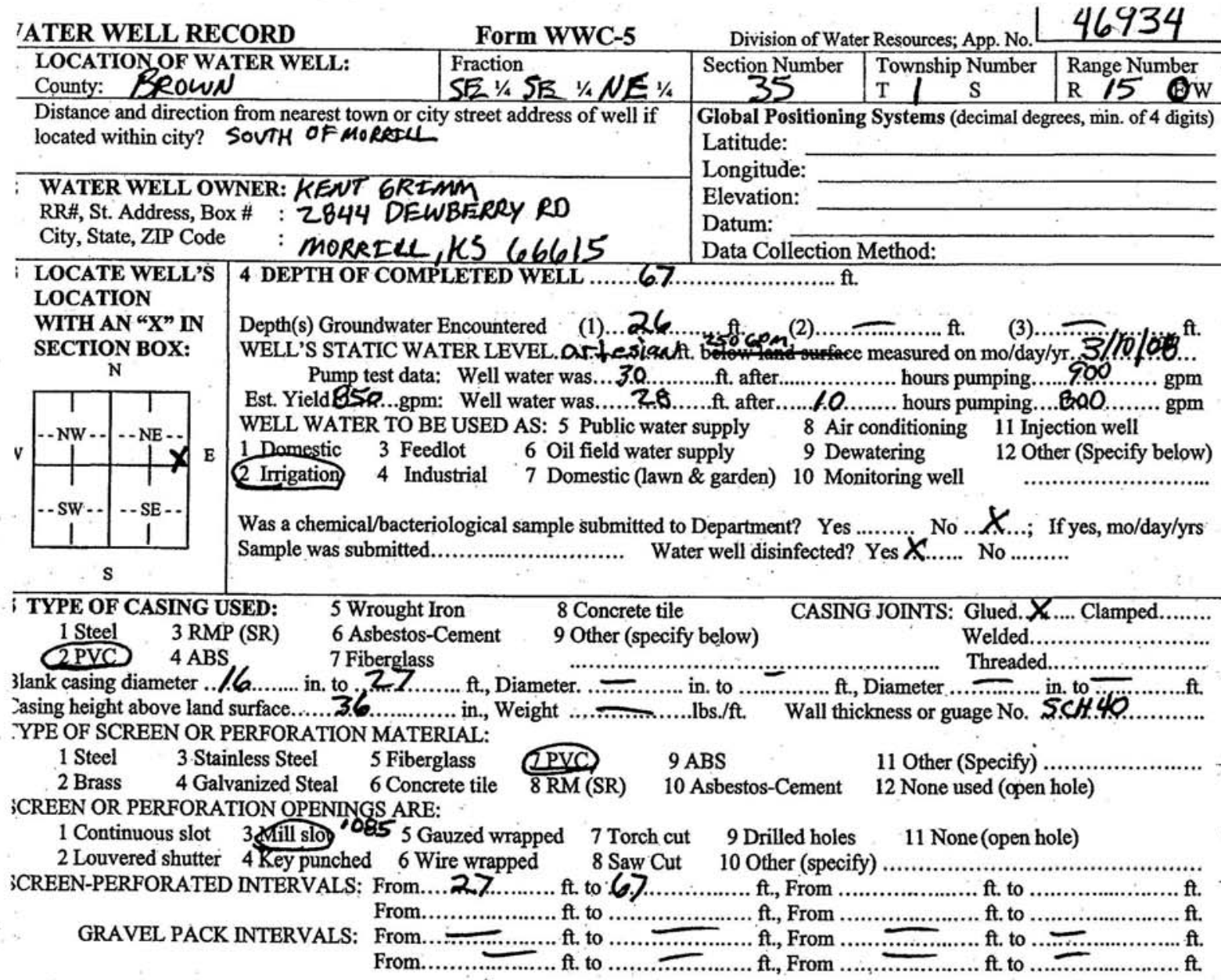

i GROUT MATERIAL: Neat cemen 2 Cement grout Bentonite 4 Other

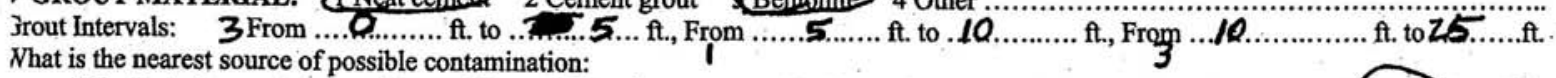

1 Septic tank

2 Sewer lines

4 Lateral lines 7 Pit privy

5 Cess pool 8 Sewage lagoon

3 Watertight sewer lines 6 Seepage pit 9 Feedyard

10 Livestock pens

11 Fuel storage

13 Insecticide Storage

14 Abandoned water well

12 Fertilizer Storage 15 Oil well/gas well

Qther specify

below)

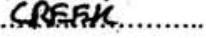

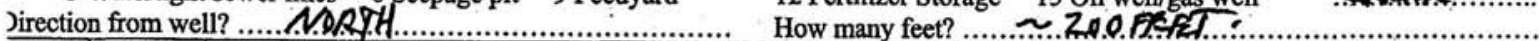

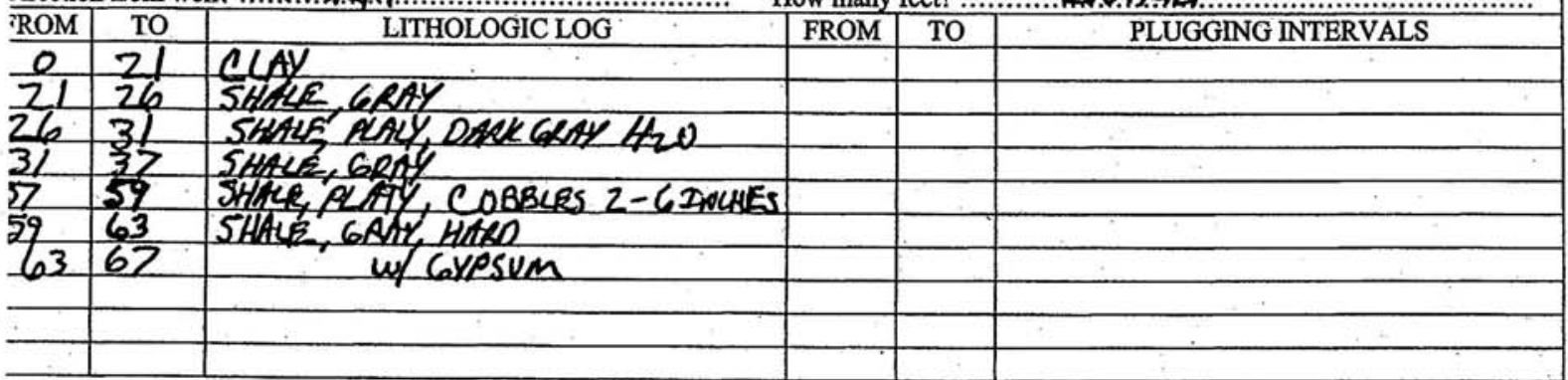

ICONTRACTOR'S OR LANDOWNER'S CERTIFICATIQN This water well was (1) constructed, (2) reconstructed, or (3) plugged under my jurisdiction and was completed on (mo/day/year). $3 / 1,0 / 0 . \mathrm{b} . . . . .$. and this record is true to the best of my knowledge and belief. Kansas Water Well Contractor's License No. .76l........ This Water Well Record was completed on (p/day/fear) $45 / .0 \% / .98$. inder the business name of ASSOCF TKO DRT LCEKC TNC. by (signature)

NSTRUCTIONS: Use typewriter or ball point pen. PLEASE PRESS FIRMMY and PRINT clearly. Please fill in blanks, finderlife or circle the correct answers. Send top hree copies to Kansas Department of Health and Environment, Bureau of Water, Geology Section, 1000 SW Jackson St., Suite 420, Topeka, Kansas 66612-1367. Telephone 85-296-5522. Send one to WATER WELL OWNER and retain one for your records. Fee of $\$ 5.00$. for each constructed well. Visit us at ttp://www.kdheks.gov/waterwell/index.html. 


\section{Appendix B:}

Sequence of Sampling Activities at Morrill, Kansas, in April-May 2008 
TABLE B.1 Sequence of sampling activities at Morrill in April-May 2008.

\begin{tabular}{|c|c|c|c|c|c|c|c|}
\hline $\begin{array}{l}\text { Sample } \\
\text { Date }\end{array}$ & Time & Location & Sample & Medium & $\begin{array}{l}\text { Depth } \\
\text { (ft TOC) }\end{array}$ & $\begin{array}{l}\text { Chain of } \\
\text { Custody No. }\end{array}$ & Description \\
\hline $04 / 14 / 08$ & $11: 53$ & MW4S & MRMW4S-W-23234 & Water & $17-47$ & 4783 & $\begin{array}{l}\text { Depth to water from top of casing }(\mathrm{TOC})=26.32 \mathrm{ft} \text {. Depth of } 4 \text {-in. } \\
\text { well }=47.85 \mathrm{ft} \text { TOC. Sample collected by using low-flow bladder } \\
\text { pump after purging of } 2.48 \mathrm{~L} \text {. Purge water clear. }\end{array}$ \\
\hline $04 / 14 / 08$ & 13:29 & MW3S & MRMW3S-W-23233 & Water & $18-48$ & 4783 & $\begin{array}{l}\text { Depth to water }=16.95 \mathrm{ft} \mathrm{TOC} \text {. Depth of } 4 \text {-in. well }=47.80 \mathrm{ft} \mathrm{TOC} \text {. } \\
\text { Sample collected by using low-flow bladder pump after purging } \\
\text { of } 3.31 \mathrm{~L} \text {. Purge water clear. }\end{array}$ \\
\hline $04 / 14 / 08$ & 13:31 & MW3S & MRMW3S-W-23246 & Water & $18-48$ & 4783 & Replicate of sample MRMW3S-W-23233. \\
\hline $04 / 14 / 08$ & $13: 40$ & QC & MRQCIR-W-23252 & Water & -- & 4783 & $\begin{array}{l}\text { Rinsate of decontaminated bladder hose after collection of sample } \\
\text { MRMW3S-W-23233 and replicate MRMW3S-W-23246. }\end{array}$ \\
\hline 04/14/08 & $14: 42$ & SMB & MRSMB-S-23258 & Soil & -- & 4782 & Sediment sample from Terrapin Creek. \\
\hline $04 / 14 / 08$ & $14: 43$ & SMB & MRSMB-W-23258 & Water & -- & 4784 & Water sample from Terrapin Creek. \\
\hline $04 / 14 / 08$ & $14: 51$ & SM1 & MRSM1-S-23254 & Soil & -- & 4782 & Sediment sample from Terrapin Creek. \\
\hline $04 / 14 / 08$ & $14: 52$ & SM1 & MRSM1-W-23254 & Water & -- & 4784 & Water sample from Terrapin Creek. \\
\hline $04 / 14 / 08$ & $14: 57$ & SM2 & MRSM2-S-23255 & Soil & -- & 4782 & Sediment sample from Terrapin Creek. \\
\hline $04 / 14 / 08$ & $14: 58$ & SM2 & MRSM2-W-23255 & Water & -- & 4784 & Water sample from Terrapin Creek. \\
\hline $04 / 14 / 08$ & $15: 03$ & SM3 & MRSM3-S-23256 & Soil & -- & 4782 & Sediment sample from Terrapin Creek. \\
\hline $04 / 14 / 08$ & $15: 04$ & SM3 & MRSM3-W-23256 & Water & -- & 4784 & Water sample from Terrapin Creek. \\
\hline $04 / 14 / 08$ & $15: 14$ & SM4 & MRSM4-S-23257 & Soil & -- & 4782 & Sediment sample from Terrapin Creek. \\
\hline $04 / 14 / 08$ & $15: 15$ & SM4 & MRSM4-W-23257 & Water & -- & 4784 & Water sample from Terrapin Creek. \\
\hline $04 / 14 / 08$ & $16: 34$ & MW1D & MRMW1D-W-23231 & Water & $63-88$ & 4783 & $\begin{array}{l}\text { Depth to water }=29.51 \mathrm{ft} \mathrm{TOC} \text {. Depth of } 4 \text {-in. well }=89.00 \mathrm{ft} \mathrm{TOC.} \\
\text { Sample collected by using low-flow bladder pump after purging } \\
\text { of } 6 \mathrm{~L} \text {. Purge water clear. Iron sample light orange. }\end{array}$ \\
\hline $04 / 14 / 08$ & $16: 41$ & MW10S & MRMW10S-W-23240 & Water & $30-45$ & 4783 & $\begin{array}{l}\text { Depth to water }=9.82 \mathrm{ft} \mathrm{TOC} \text {. Depth of } 2 \text {-in. well }=49.70 \mathrm{ft} \mathrm{TOC} \text {. } \\
\text { Sample collected by using low-flow bladder pump after purging } \\
\text { of } 1.90 \mathrm{~L} \text {. Purge water tan color. }\end{array}$ \\
\hline $04 / 14 / 08$ & $17: 12$ & MW1S & MRMW1S-W-23230 & Water & $11-51$ & 4783 & $\begin{array}{l}\text { Depth to water }=16.20 \mathrm{ft} \mathrm{TOC} \text {. Depth of } 4 \text {-in. well }=54.00 \mathrm{ft} \text { TOC. } \\
\text { Sample collected by using low-flow bladder pump after purging } \\
\text { of } 5.5 \mathrm{~L} \text {. Purge water clear. }\end{array}$ \\
\hline 04/14/08 & $17: 14$ & MW1S & MRMW1S-W-23245 & Water & $11-51$ & 4783 & Replicate of sample MRMW1S-W-23230. \\
\hline $04 / 14 / 08$ & $17: 34$ & QC & MRQCIR-W-23251 & Water & -- & 4783 & $\begin{array}{l}\text { Rinsate of decontaminated bladder hose after collection of sample } \\
\text { MRMW1S-W-23230 and replicate MRMW1S-W-23245. }\end{array}$ \\
\hline $04 / 14 / 08$ & $18: 15$ & MW7S & MRMW7S-W-23237 & Water & $20-45$ & 4783 & $\begin{array}{l}\text { Depth to water }=7.72 \mathrm{ft} \mathrm{TOC} \text {. Depth of } 4 \text {-in. well }=47.00 \mathrm{ft} \mathrm{TOC} \text {. } \\
\text { Sample collected by using low-flow bladder pump after purging } \\
\text { of } 1.82 \mathrm{~L} \text {. Purge water clear. }\end{array}$ \\
\hline $04 / 14 / 08$ & $18: 16$ & MW5S & MRMW5S-W-23235 & Water & $15-55$ & 4783 & $\begin{array}{l}\text { Depth to water }=11.20 \mathrm{ft} \mathrm{TOC} \text {. Depth of } 4 \text {-in. well }=54.60 \mathrm{ft} \mathrm{TOC} \text {. } \\
\text { Sample collected by using low-flow bladder pump after purging } \\
\text { of } 6 \mathrm{~L} \text {. Purge water clear. }\end{array}$ \\
\hline $04 / 14 / 08$ & $18: 17$ & MW7S & MRMW7S-W-23247 & Water & $20-45$ & 4783 & Replicate of sample MRMW7S-W-23237. \\
\hline
\end{tabular}

Replicate of sample MRMW7S-W-23237. 
TABLE B.1 (Cont.)

\begin{tabular}{|c|c|c|c|c|c|c|c|}
\hline $\begin{array}{l}\text { Sample } \\
\text { Date }\end{array}$ & Time & Location & Sample & Medium & $\begin{array}{l}\text { Depth } \\
\text { (ft TOC) }\end{array}$ & $\begin{array}{l}\text { Chain of } \\
\text { Custody No. }\end{array}$ & Description \\
\hline $04 / 14 / 08$ & $18: 22$ & $Q C$ & MRQCIR-W-23253 & Water & -- & 4783 & $\begin{array}{l}\text { Rinsate of decontaminated bladder hose after collection of } \\
\text { sample MRMW7S-W-23237 and replicate MRMW7S-W-23247. }\end{array}$ \\
\hline $04 / 14 / 08$ & $19: 24$ & MW9S & MRMW9S-W-23239 & Water & $38.83-53.83$ & 4783 & $\begin{array}{l}\text { Depth to water }=16.58 \mathrm{ft} \text { TOC. Depth of } 2 \text {-in. well }=58.63 \mathrm{ft} \mathrm{TOC} \text {. } \\
\text { Sample collected by using low-flow bladder pump after purging } \\
\text { of } 2.29 \mathrm{~L} \text {. Purge water dirty looking. }\end{array}$ \\
\hline $04 / 15 / 08$ & $7: 56$ & MW6S & MRMW6S-W-23236 & Water & $10-25$ & 4784 & $\begin{array}{l}\text { Depth to water }=5.15 \mathrm{ft} \text { TOC. Depth of } 4 \text {-in. well }=26.90 \mathrm{ft} \mathrm{TOC} \text {. } \\
\text { Sample collected by using low-flow bladder pump after purging } \\
\text { of } 2.5 \mathrm{~L} \text {. Purge water clear. Iron sample light orange color. }\end{array}$ \\
\hline $04 / 15 / 08$ & $9: 14$ & MW8S & MRMW8S-W-23238 & Water & $10-25$ & 4784 & $\begin{array}{l}\text { Depth to water }=0.70 \mathrm{ft} \mathrm{TOC} \text {. Depth of } 4 \text {-in. well }=26.80 \mathrm{ft} \mathrm{TOC} \text {. } \\
\text { Sample collected by using low-flow bladder pump after purging } \\
\text { of } 5.5 \mathrm{~L} \text {. Purge water clear. }\end{array}$ \\
\hline 04/15/08 & $9: 32$ & MW2S & MRMW2S-W-23232 & Water & $13-53$ & 4784 & $\begin{array}{l}\text { Depth to water }=23.55 \mathrm{ft} \text { TOC. Depth of } 4 \text {-in. well }=53.40 \mathrm{ft} \mathrm{TOC} \text {. } \\
\text { Sample collected by using low-flow bladder pump after purging } \\
\text { of } 2.22 \mathrm{~L} \text {. Purge water clear. }\end{array}$ \\
\hline $04 / 15 / 08$ & $10: 28$ & MW11S & MRMW11S-W-23241 & Water & $53-68$ & 4784 & $\begin{array}{l}\text { Depth to water }=29.90 \mathrm{ft} \mathrm{TOC} \text {. Depth of } 2 \text {-in. well }=72.70 \mathrm{ft} \mathrm{TOC} \text {. } \\
\text { Sample collected by using low-flow bladder pump after purging } \\
\text { of } 5.5 \mathrm{~L} \text {. Purge water clear. }\end{array}$ \\
\hline $04 / 15 / 08$ & $11: 30$ & QC & MRQCTB-W-23248 & Water & -- & 4784 & $\begin{array}{l}\text { Trip blank sent to the AGEM Laboratory for organic analyses with } \\
\text { water samples listed on COCs } 4783 \text { and } 4784 \text {, as well as to } \\
\text { Envirosystems for verification organic analysis with samples } \\
\text { listed on chain-of-custody (COC) form } 4785 \text {. }\end{array}$ \\
\hline 04/15/08 & $11: 52$ & Isch & MRISCH-W-23242 & Water & -- & 4784 & $\begin{array}{l}\text { Isch well at co-op. Purge water clear. Co-op personnel turned } \\
\text { pump on to purge and sample. }\end{array}$ \\
\hline $04 / 15 / 08$ & $12: 08$ & Rilinger & MRRILINGER-W-23243 & Water & - & 4784 & $\begin{array}{l}\text { Rilinger well. Purge water clear. Turned well on for five minutes, } \\
\text { then sampled. }\end{array}$ \\
\hline $04 / 15 / 08$ & $12: 16$ & Stone & MRSTONE-W-23244 & Water & 43 & 4784 & $\begin{array}{l}\text { Stone well. Purge water clear. Water level tape hit bottom at } \\
38.86 \mathrm{ft} \text { TOC and became caught on obstruction when } \\
\text { removed. Bladder pump hit bottom at } 32.84 \mathrm{ft} \text { TOC. Sample } \\
\text { collected by using bailer. }\end{array}$ \\
\hline $04 / 22 / 08$ & $10: 20$ & TD12 & MRTD12-W-23264 & Water & -- & 4786 & $\begin{array}{l}\text { Sample from Grimm irrigation well discharge for organic analyses } \\
\text { at the AGEM Laboratory. }\end{array}$ \\
\hline $04 / 22 / 08$ & $11: 16$ & Isch & MRISCH-W-23262 & Water & -- & 4786 & $\begin{array}{l}\text { Resampling to confirm apparent effect of Grimm irrigation well on } \\
\text { plume contamination. }\end{array}$ \\
\hline 04/22/08 & $11: 40$ & QC & MRQCTB-W-23263 & Water & -- & 4786 & $\begin{array}{l}\text { Trip blank sent to the AGEM Laboratory for organic analyses with } \\
\text { water samples listed on COC 4786, as well as to } \\
\text { Envirosystems for verification organic analysis with samples } \\
\text { listed on COC } 4787 \text {. }\end{array}$ \\
\hline
\end{tabular}


TABLE B.1 (Cont.)

\begin{tabular}{|c|c|c|c|c|c|c|c|}
\hline $\begin{array}{l}\text { Sample } \\
\text { Date }\end{array}$ & Time & Location & Sample & Medium & $\begin{array}{l}\text { Depth } \\
\text { (ft TOC) }\end{array}$ & $\begin{array}{l}\text { Chain of } \\
\text { Custody No. }\end{array}$ & Description \\
\hline 04/22/08 & $12: 52$ & MW1S & MRMW1S-W-23259 & Water & $11-51$ & 4786 & $\begin{array}{l}\text { Resampling to confirm apparent effect of Grimm irrigation well on } \\
\text { plume contamination. Depth to water }=16.00 \mathrm{ft} \text { TOC. Depth of } \\
4 \text {-in. well }=54.00 \mathrm{ft} \text { TOC. Sample collected by using low-flow } \\
\text { bladder pump after purging of } 6.25 \mathrm{~L} \text {. Purge water clear. }\end{array}$ \\
\hline 04/22/08 & $13: 46$ & MW3S & MRMW3S-W-23260 & Water & $18-48$ & 4786 & $\begin{array}{l}\text { Resampling to confirm apparent effect of Grimm irrigation well on } \\
\text { plume contamination. Depth to water }=15.80 \mathrm{ft} \mathrm{TOC} \text {. Depth of } \\
4 \text {-in. well }=47.80 \mathrm{ft} \mathrm{TOC.} \mathrm{Sample} \mathrm{collected} \mathrm{by} \mathrm{using} \mathrm{low-flow} \\
\text { bladder pump after purging of } 6.5 \mathrm{~L} \text {. Purge water clear. }\end{array}$ \\
\hline 04/22/08 & $15: 08$ & MW11S & MRMW11S-W-23261 & Water & $53-68$ & 4786 & $\begin{array}{l}\text { Resampling to confirm apparent effect of Grimm irrigation well on } \\
\text { plume contamination. Depth to water }=30.20 \mathrm{ft} \mathrm{TOC} \text {. Depth of } \\
2 \text {-in. well }=72.70 \mathrm{ft} \mathrm{TOC} \text {. Sample collected by using low-flow } \\
\text { bladder pump after purging of } 7.2 \mathrm{~L} \text {. Purge water cloudy to } \\
\text { clear. }\end{array}$ \\
\hline $04 / 23 / 08$ & $16: 46$ & MW7S & MRMW7S-W-23265 & Water & $20-45$ & 4789 & $\begin{array}{l}\text { Resampling to confirm apparent effect of Grimm irrigation well on } \\
\text { plume contamination. Depth to water }=7.80 \mathrm{ft} \mathrm{TOC} \text {. Depth of } \\
4 \text {-in. well }=47.00 \mathrm{ft} \mathrm{TOC.} \mathrm{Sample} \mathrm{collected} \mathrm{by} \mathrm{using} \mathrm{low-flow} \\
\text { bladder pump after purging of } 11 \mathrm{~L} \text {. Purge water cloudy to } \\
\text { clear. }\end{array}$ \\
\hline $04 / 23 / 08$ & $17: 50$ & MW5S & MRMW5S-W-23266 & Water & $15-55$ & 4789 & $\begin{array}{l}\text { Resampling to confirm apparent effect of Grimm irrigation well on } \\
\text { plume contamination. Depth to water }=11.30 \mathrm{ft} \text { TOC. Depth of } \\
4 \text {-in. well }=54.60 \mathrm{ft} \text { TOC. Sample collected by using low-flow } \\
\text { bladder pump after purging of } 6.5 \mathrm{~L} \text {. }\end{array}$ \\
\hline 04/23/08 & $18: 30$ & TD12 & MRTD12-W-23267 & Water & -- & 4792 & $\begin{array}{l}\text { Sample collected from Grimm irrigation well discharge for anions, } \\
\text { cations, and VOCs analyses by TestAmerica. }\end{array}$ \\
\hline $04 / 23 / 08$ & $18: 45$ & QC & MRQCTB-W-23268 & Water & -- & 4789 & $\begin{array}{l}\text { Trip blank sent to the AGEM Laboratory for organic analyses with } \\
\text { water samples listed on COC 4789, as well as to } \\
\text { Envirosystems for verification organic analysis with samples } \\
\text { listed on COC } 4790 \text { and to TestAmerica for verification organic } \\
\text { analysis with samples listed on COC } 4792 \text {. }\end{array}$ \\
\hline 05/01/08 & $6: 25$ & MW3S & MRMW3S-45-W-23271 & Water & $18-48$ & 6060 & $\begin{array}{l}\text { Bladder pump intake at } 45 \mathrm{ft} \mathrm{TOC} \text {. Purge water clear. Sample } \\
\text { collected after purging of } 3.17 \mathrm{~L} \text {. }\end{array}$ \\
\hline $05 / 01 / 08$ & $6: 59$ & MW3S & MRMW3S-38-W-23270 & Water & $18-48$ & 6060 & $\begin{array}{l}\text { Bladder pump intake at } 38 \mathrm{ft} \mathrm{TOC.} \mathrm{Purge} \mathrm{water} \mathrm{clear.} \mathrm{Sample} \\
\text { collected after purging of } 2.75 \mathrm{~L} \text {. }\end{array}$ \\
\hline 05/01/08 & $7: 37$ & MW3S & MRMW3S-26-W-23269 & Water & $18-48$ & 6060 & $\begin{array}{l}\text { Bladder pump intake at } 26 \mathrm{ft} \text { TOC. Purge water clear. Sample } \\
\text { collected after purging of } 2.62 \mathrm{~L} \text {. }\end{array}$ \\
\hline 05/01/08 & 9:00 & MW5S & MRMW5S-52-W-23274 & Water & $15-55$ & 6060 & $\begin{array}{l}\text { Bladder pump intake at } 52 \mathrm{ft} \mathrm{TOC.} \mathrm{Purge} \mathrm{water} \mathrm{clear.} \mathrm{Sample} \\
\text { collected after purging of } 4 \mathrm{~L} \text {. }\end{array}$ \\
\hline
\end{tabular}


TABLE B.1 (Cont.)

\begin{tabular}{|c|c|c|c|c|c|c|c|}
\hline $\begin{array}{l}\text { Sample } \\
\text { Date }\end{array}$ & Time & Location & Sample & Medium & $\begin{array}{l}\text { Depth } \\
\text { (ft TOC) }\end{array}$ & $\begin{array}{l}\text { Chain of } \\
\text { Custody No. }\end{array}$ & Description \\
\hline $05 / 01 / 08$ & $9: 36$ & MW5S & MRMW5S-28-W-23273 & Water & $15-55$ & 6060 & $\begin{array}{l}\text { Bladder pump intake at } 28 \mathrm{ft} \mathrm{TOC} \text {. Purge water clear. Sample } \\
\text { collected after purging of } 3.4 \mathrm{~L} \text {. }\end{array}$ \\
\hline $05 / 01 / 08$ & $10: 18$ & MW5S & MRMW5S-20-W-23272 & Water & $15-55$ & 6060 & $\begin{array}{l}\text { Bladder pump intake at } 20 \mathrm{ft} \mathrm{TOC} \text {. Purge water clear. Sample } \\
\text { collected after purging of } 3.7 \mathrm{~L} \text {. }\end{array}$ \\
\hline 05/01/08 & $11: 34$ & MW1S & MRMW1S-48-W-23277 & Water & $11-51$ & 6060 & $\begin{array}{l}\text { Bladder pump intake at } 48 \mathrm{ft} \mathrm{TOC} \text {. Purge water clear. Sample } \\
\text { collected after purging of } 4.25 \mathrm{~L} \text {. }\end{array}$ \\
\hline $05 / 01 / 08$ & $12: 15$ & MW1S & MRMW1S-27-W-23276 & Water & $11-51$ & 6060 & $\begin{array}{l}\text { Bladder pump intake at } 27 \mathrm{ft} \mathrm{TOC} \text {. Purge water clear. Sample } \\
\text { collected after purging of } 3.24 \mathrm{~L} \text {. }\end{array}$ \\
\hline 05/01/08 & $12: 53$ & MW1S & MRMW1S-22-W-23275 & Water & $11-51$ & 6060 & $\begin{array}{l}\text { Bladder pump intake at } 22 \mathrm{ft} \mathrm{TOC} \text {. Purge water clear. Sample } \\
\text { collected after purging of } 3.24 \mathrm{~L} \text {. }\end{array}$ \\
\hline 05/01/08 & $13: 29$ & QC & MRQCTB-W-23278 & Water & - & 6060 & $\begin{array}{l}\text { Trip blank sent to the AGEM Laboratory for organic analyses with } \\
\text { water samples listed on COC } 6060 \text {. }\end{array}$ \\
\hline
\end{tabular}




\section{Appendix C:}

\section{Analytical Results for Waste Purge Water}


May 27, 2008

\section{Lisa Larsen}

Larsen \& Associates, Inc.

1311 E. 25th St.

Suite $B$

Lawrence, KS 66046

RE: Project: Morrill-ANL

Pace Project No.: 6040555

Dear Lisa Larsen:

Enclosed are the analytical results for sample(s) received by the laboratory on May 21,2008 . The results relate only to the samples included in this report. Results reported herein conform to the most current NELAC standards, where applicable, unless otherwise narrated in the body of the report.

If you have any questions concerning this report, please feel free to contact me.

Sincerely,

$$
\text { suraty sijoson }
$$

Trudy Gipson

trudy.gipson@pacelabs.com

Project Manager.

A2LA Certification Number: 2456.01

Arkansas Certification Number: 05-008-0

Ilinois Certification Number: 001191

lowa Certification Number: 118

Kansas/NELAP Certification Number: E-10116

Louisiana Certification Number: 03055

Oklahoma Certification Number: 9205/9935

Utah Certification Number: 9135995665

Enclosures

cc: Nadine Appenbrink, Larsen \& Associates, Inc.

Mike Dinkel, Larsen \& Associates, Inc. 


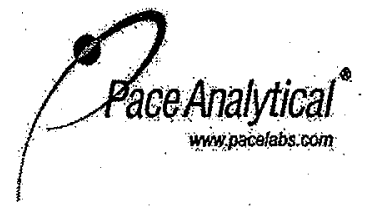

Pace Analytical Services, Inc. 9608 Loiret Blvd. Lenexa, KS 66219 (913)599-5665

May 27, 2008

Page 2

cc: Brooke Evans, Larsen \& Associates, Inc. 


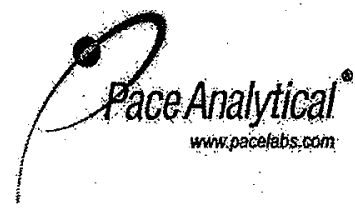

Pace Analytical Services, Inc. 9608 Loiret Blvd. Lenexa, KS 66219 (913)599-5665

SAMPLE SUMMARY

$\begin{array}{ll}\text { Project: } & \text { Morrill-ANL } \\ \text { Pace Project No.: } & 6040555\end{array}$

\begin{tabular}{llllll}
\hline Lab ID & Sample ID & Matrix & Date Collected & Date Received \\
\hline 6040555001 & PURGE WATER & Water & $05 / 21 / 0811: 00$ & $05 / 21 / 0815: 26$ \\
6040555002 & TRIP BLANK & Water & $05 / 21 / 0800: 00$ & $05 / 21 / 0815: 26$
\end{tabular}




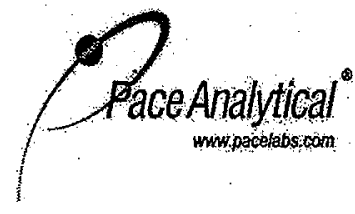
Pace Analytical Seivices, Inc.
9608 Loiret Bivd.
Lenexa, KS 66219
(913)599-5665

SAMPLE ANALYTE COUNT

$\begin{array}{ll}\text { Project: } & \text { Morrill-ANL } \\ \text { Pace Project No.: } & 6040555\end{array}$

\begin{tabular}{|c|c|c|c|c|}
\hline Lab ID & Sample ID & Method & Analysts & $\begin{array}{l}\text { Analytes } \\
\text { Reported }\end{array}$ \\
\hline 6040555001 & PURGE WATER & EPA $5030 B / 8260$ & JTK & 70 \\
\hline & & EPA 504.1 & $\mathrm{CDI}$ & 1 \\
\hline 6040555002 & TRIP BLANK & EPA 5030B/8260 & JTK & 70 \\
\hline
\end{tabular}




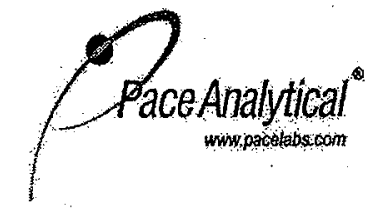

Pace Analytical Services, Inc. 9608 Loiret Blvd. Lenexa, KS 66219 (913)599-5665

\section{ANALYTICAL RESULTS}

Project: Morrill-ANL

Pace Project No.: 6040555

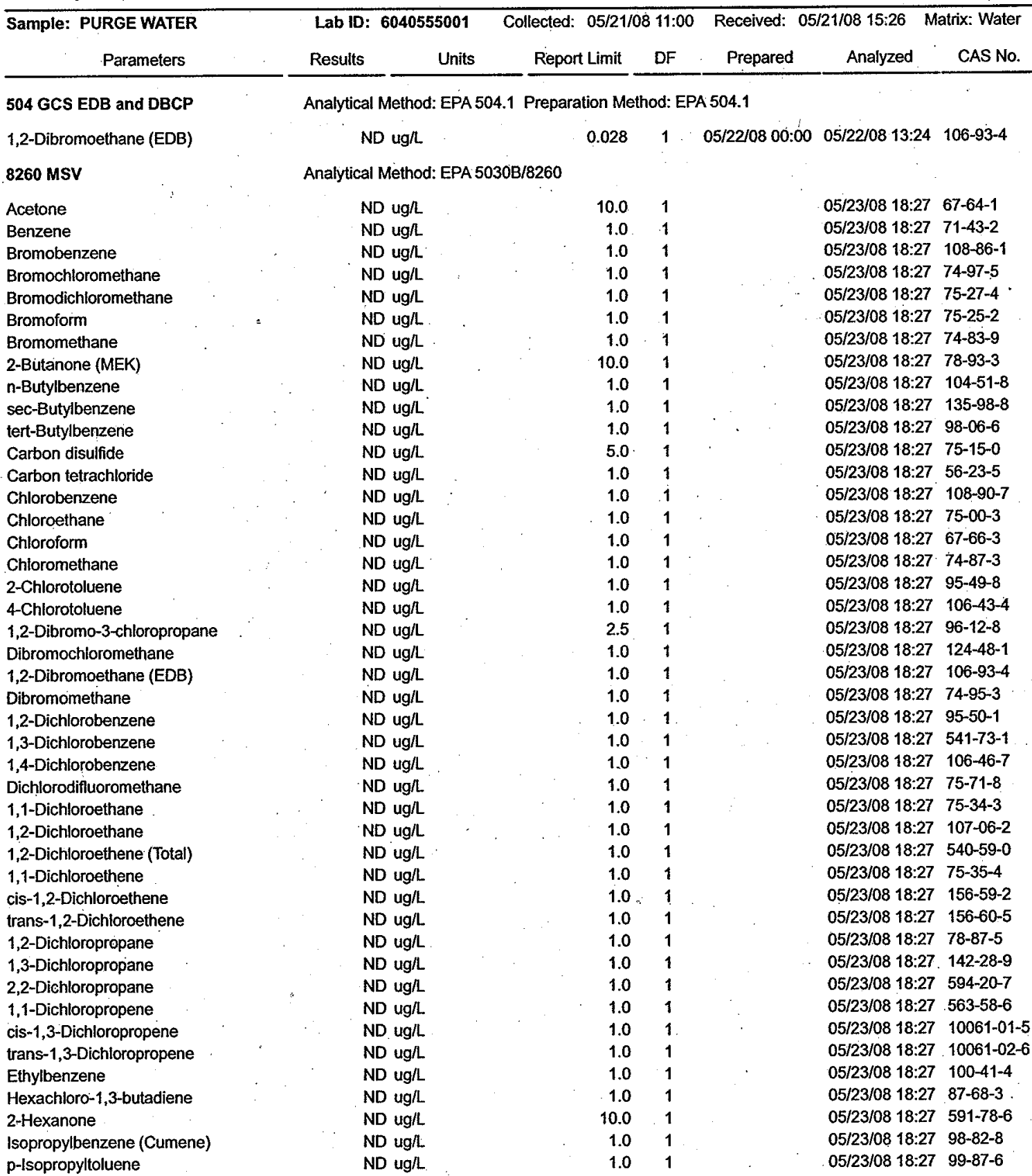

\section{REPORT OF LABORATORY ANALYSIS}




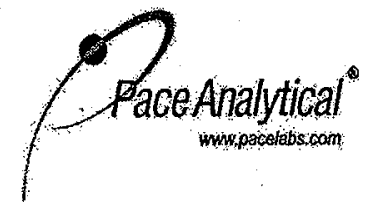

Pace Analytical Services, Inc. 9608 Loiret Blvd. Lenexa, KS 66219 (913)599-5665

\section{ANALYTICAL RESULTS}

Project: Morrill-ANL

Pace Project No:: 6040555

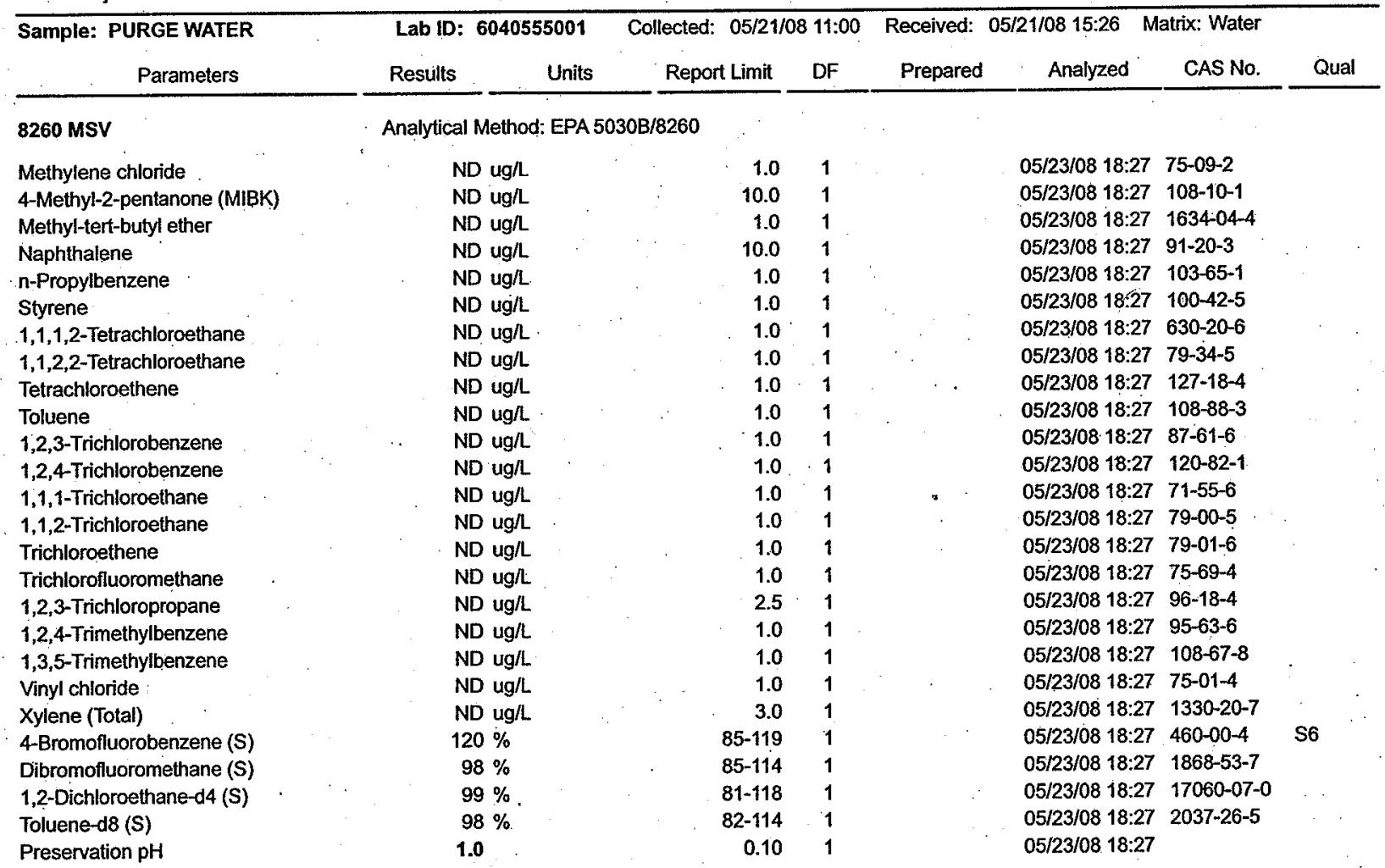




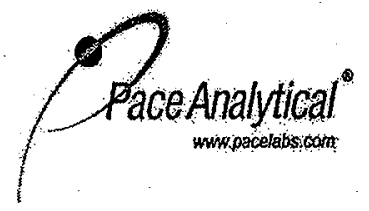

Pace Analytical Services, Inc.

9608 Loiret Blvd.

Lenexa, KS 66219

(913)599-5665

ANALYTICAL RESULTS

Project: Morrill-ANL

Pace Project No:: 6040555

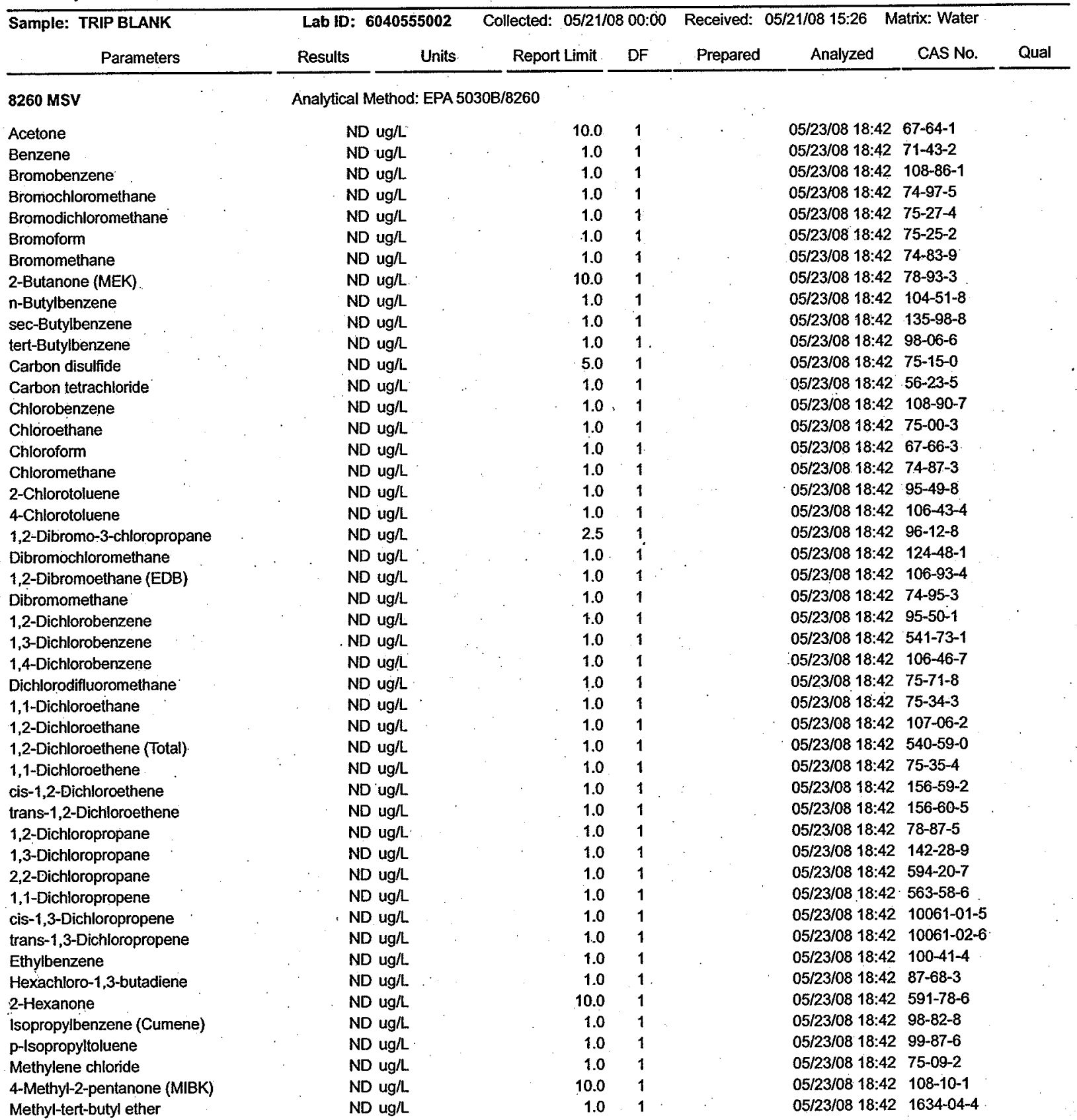

Date: 05/27/2008 02:26 PM

REPORT OF LABORATORY ANALYSIS

Page 7 of 15

This report shall not be reproduced, except in full,

without the witten consent of Pace Analytical Services, Inc.

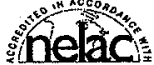




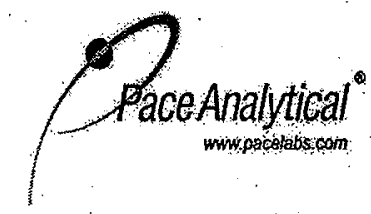

Pace Analytical Services, Inc.

9608 Loiret Blvd.

Lenexa, KS 66.219

(913)599-5665

\section{ANALYTICAL RESULTS}

Project: Morrill-ANL

Pace Project No.: 6040555

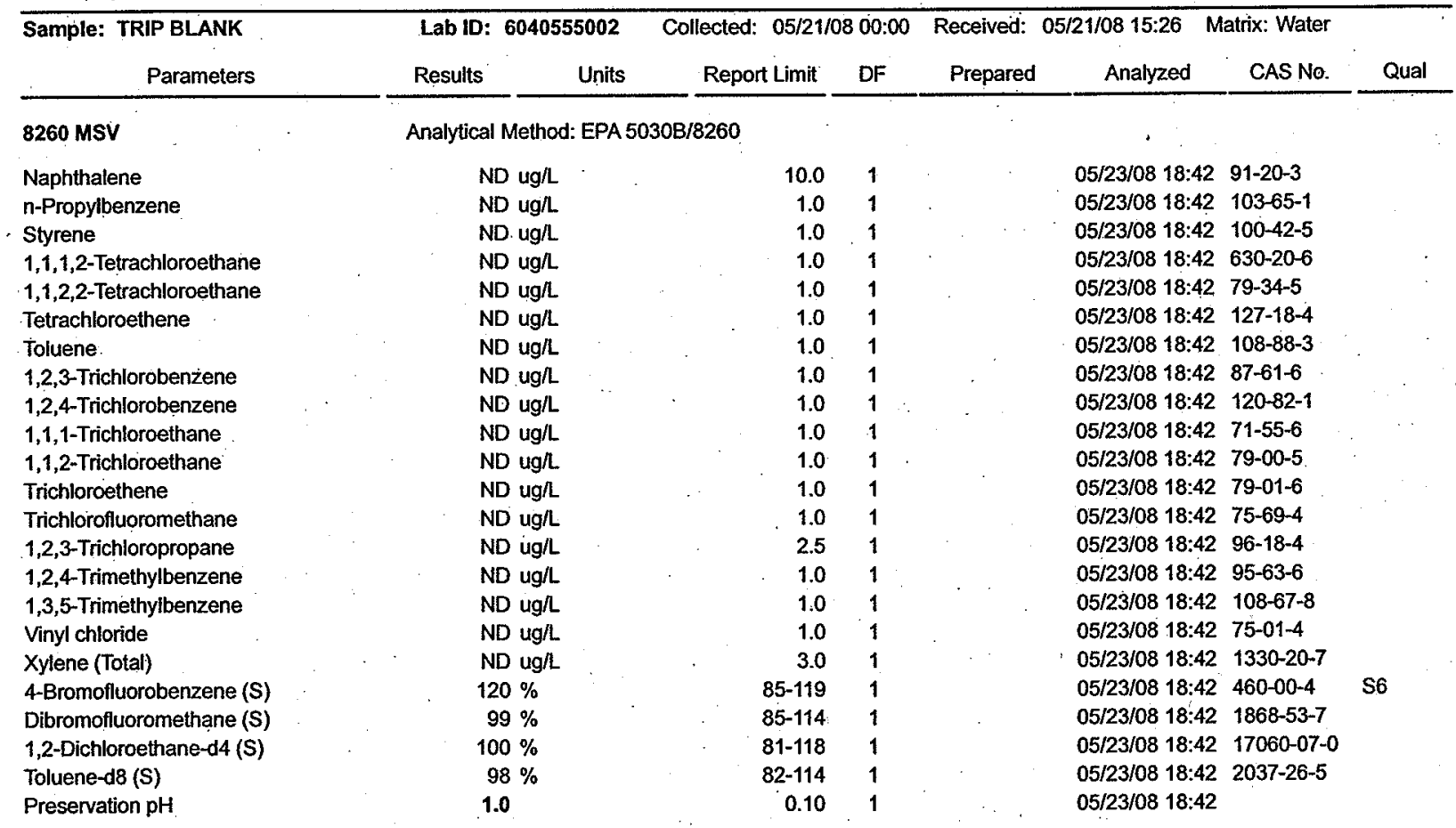




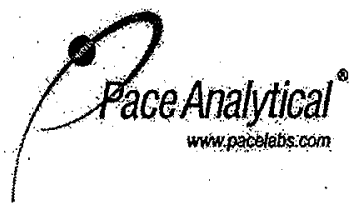

Pace Analytical Services, Inc. 9608 Loiret Blvd. Lenexa, KS 66219 (913)599-5665

QUALITY CONTROL DATA

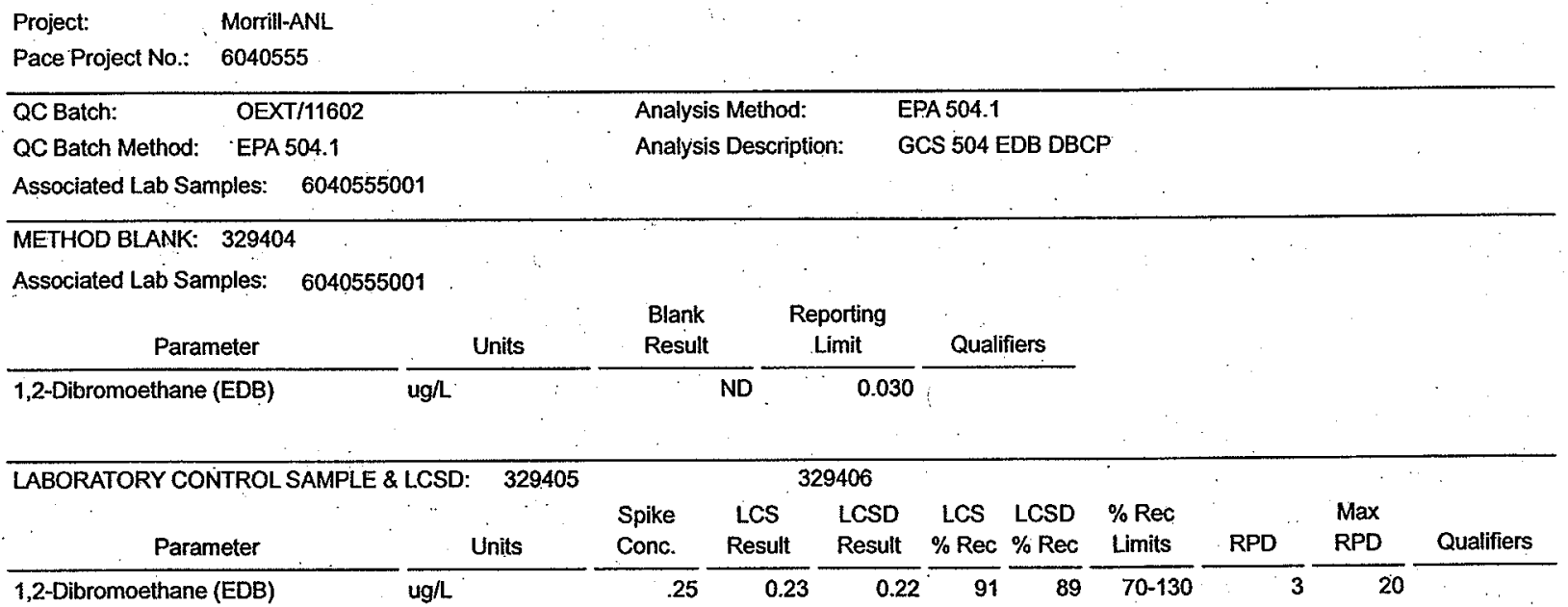




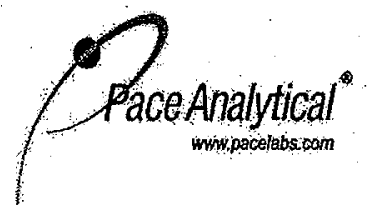

Pace Analytical Services, Inc. 9608 Loiret Blvd. Lenexa, KS 66219 (913)599-5665

\section{QUALITY CONTROL DATA}

\begin{tabular}{|c|c|c|c|}
\hline Project: & Morrill-ANL & & - \\
\hline Pace Project No.: & 6040555 & . & \\
\hline QC Batch: & MSV/14706 & Analysis Method: & EPA $5030 B / 8260$ \\
\hline QC Batch Method: & EPA $5030 B / 8260$ & Analysis Description: & 8260 MSV Water $10 \mathrm{~mL}$ Purge \\
\hline
\end{tabular}

Associated Lab Samples: $\quad 6040555001,6040555002$

METHOD BLANK: 329662
Associated Lab Samples: $\quad 6040555001,6040555002$

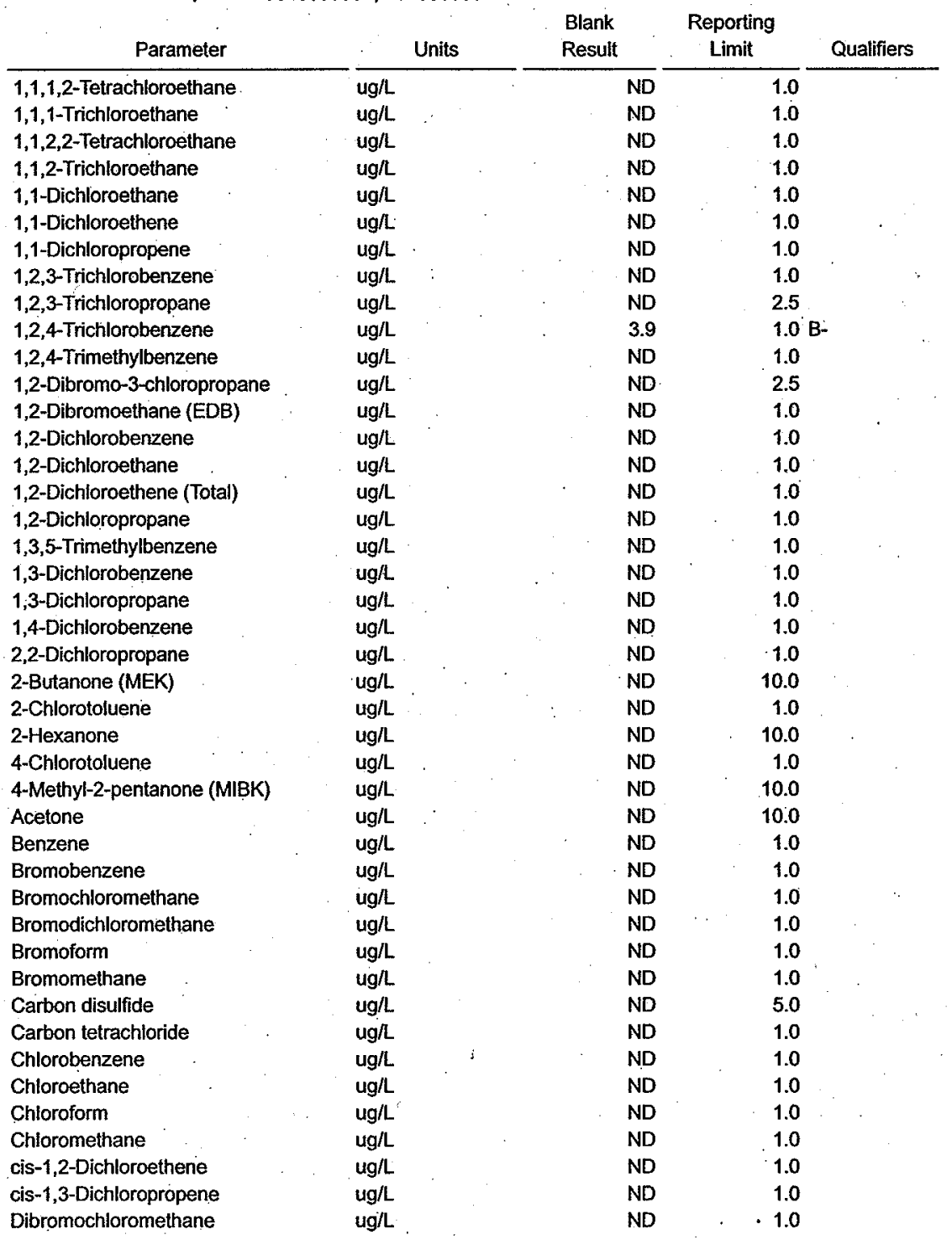

Date: 05/27/2008 02:26 PM 


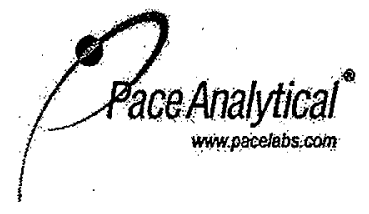

Pace Analytical Services, Inc 9608 Loiret Blvd. Lenexa, KS.66219

(913)599-5665

\section{QUALITY CONTROL DATA}

Project: Morrill-ANL

Pace Project No.: 6040555

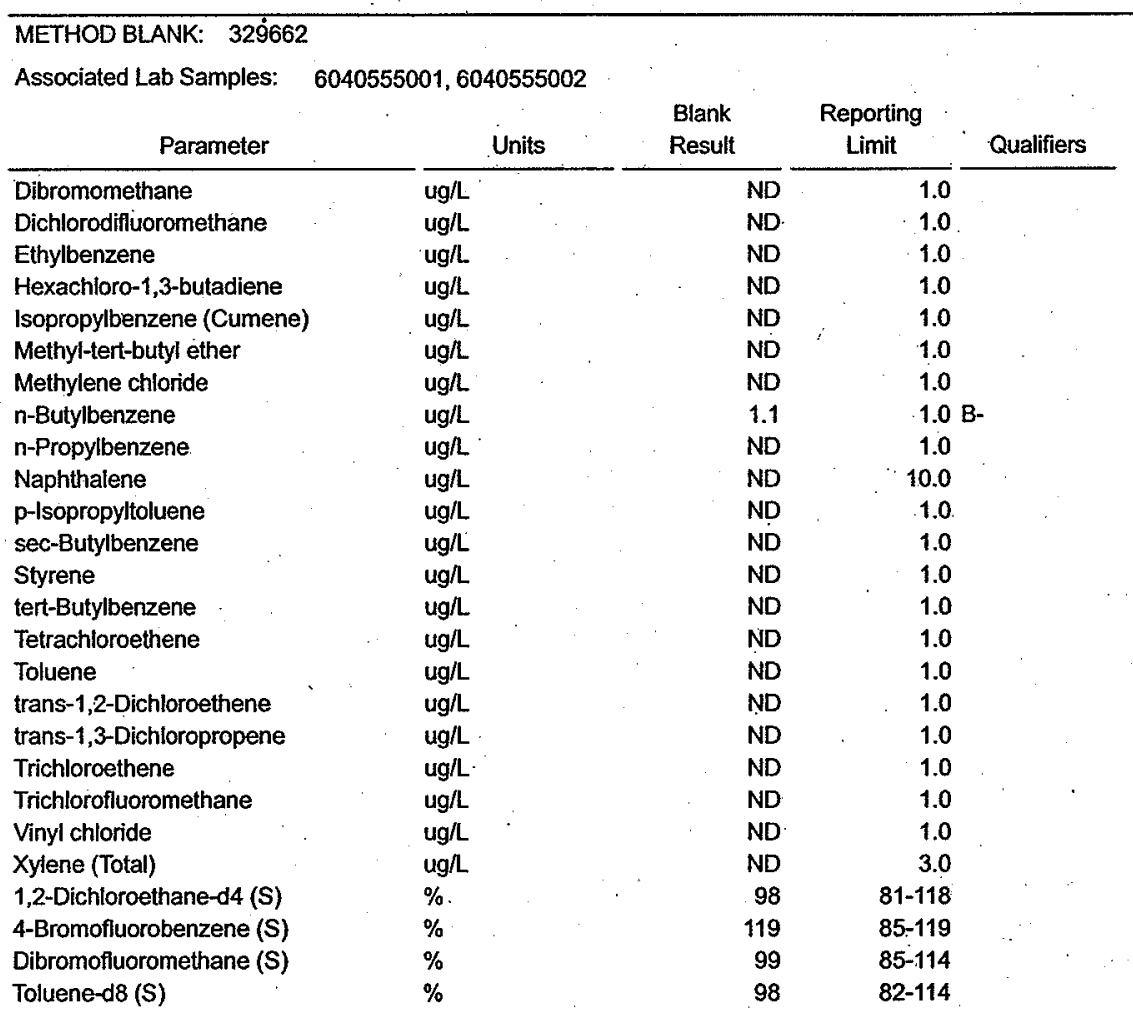

\begin{tabular}{|c|c|c|c|c|c|c|c|c|}
\hline \multirow{2}{*}{$\begin{array}{c}\text { LABORATORY CONTROL SAMPLE: } \\
\text { Parameter }\end{array}$} & \multicolumn{8}{|c|}{329663} \\
\hline & & Units & & $\begin{array}{l}\text { Spike } \\
\text { Conc. }\end{array}$ & $\begin{array}{c}\text { LCS } \\
\text { Result }\end{array}$ & $\begin{array}{c}\text { LCS } \\
\% \operatorname{Rec}\end{array}$ & $\begin{array}{l}\% \text { Rec } \\
\text { Limits }\end{array}$ & Qualifiers \\
\hline 1,1,1,2-Tetrachloroethane & ug/h & & & 10 & 10.8 & 108 & $77-127$ & \\
\hline 1,1,1-Trichloroethane & ug/L & & & 10 & 9.8 & 98 & $78-130$ & \\
\hline 1,1,2,2-Tetrachloroethane & ug/L & & . & 10 & 10.7 & 107. & $73-131$ & \\
\hline 1,1,2-Trichloroethane & $\mathrm{ug} / \mathrm{L}$ & & & 10 & 9.6 & 96 & $85-126$ & \\
\hline 1,1-Dichloroethane & ug/L & & & 10 & 9.5 & 95 & $76-124$ & \\
\hline 1,1-Dichloroethene & ug $/ \mathrm{L}$ & & & 10 & 9.7 & 97 & $76-129$ & \\
\hline 1,1-Dichloropropene & $u g / L$ & & & 10 & 9.6 & 96 & $83-125$ & \\
\hline 1,2,3-Trichlorobenzene & ug $/ \mathrm{L}$ & $\therefore$ & & $10^{\circ}$ & 11.1 & 111 & $78-129$ & \\
\hline 1,2,3-Trichloropropane & $\mathrm{ug} / \mathrm{L}$ & & & 10 & 10 & 100 & $69-117$ & \\
\hline 1,2,4-Trichlorobenzene & $\mathrm{ug} / \mathrm{L}$ & & & 10 & 11.7 & 117 & $79-127$ & \\
\hline 1,2,4-Trimethylbenzene & $\mathrm{ug} / \mathrm{L}$ & . & & 10 & 9.6 & 96 & $82-124$ & \\
\hline 1,2-Dibromo-3-chloropropane & $\mathrm{ug} / \mathrm{L}$ & & & 10 & 11.3 & 113 & $62-141$ & \\
\hline 1,2-Dibromoethane (EDB) & $u g / L$ & & & 10 & 9.7 & 97 & $85-124$ & \\
\hline 1,2-Dichlorobenzene & $u g / L$ & & $\therefore$ & 10 & 9.6 & 96 & $85-123$ & \\
\hline 1,2-Dichloroethane & $u g / L$ & & & 10 & 9.7 & 97 & $77-129$ & \\
\hline
\end{tabular}

Date: 05/27/2008 02:26 PM 


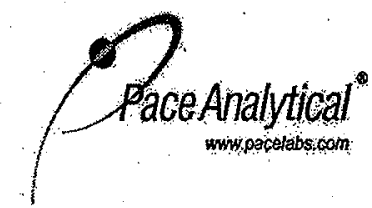

Pace Analytical Services, Inc. 9608 Loiret Blvd. Lenexa, KS 66219

(913)599-5665

QUALITY CONTROL DATA

Project: Morrill-ANL

Pace Project No:: 6040555

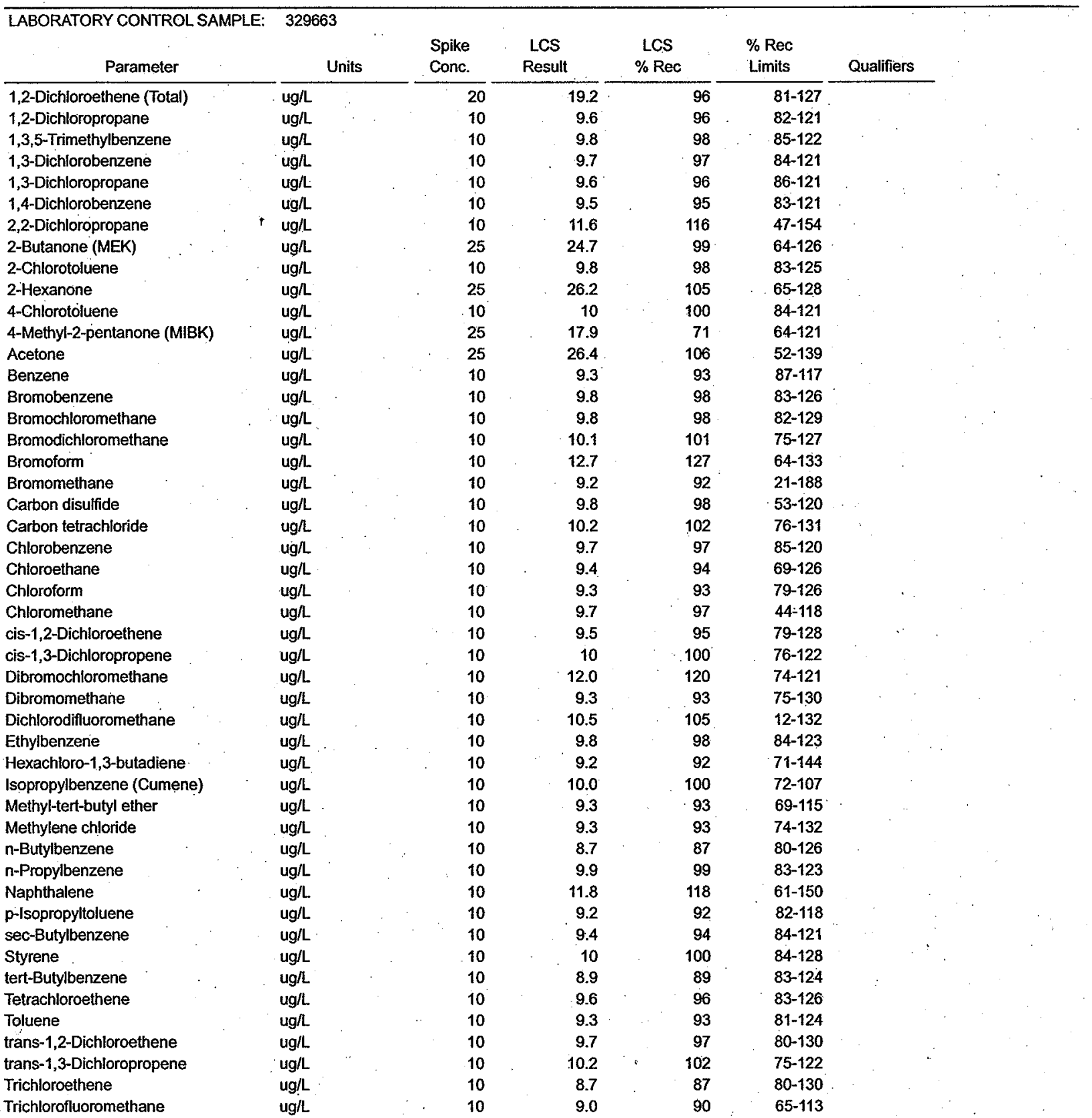

Date: 05/27/2008 02:26 PM 


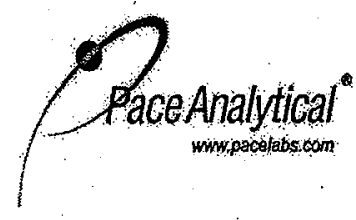

Pace Analytical Services, Inc. 9608 Loiret Blvd. Lenexa, KS 66219 (913)599-5665

QUALITY CONTROL DATA

$\begin{array}{ll}\text { Project: } & \text { Morrill-ANL } \\ \text { Pace Project No.: } & 6040555\end{array}$

LABORATORY CONTROL SAMPLE: 329663

\begin{tabular}{|c|c|c|c|c|c|c|c|}
\hline Parameter. & $\because$ & & $\begin{array}{l}\text { Spike } \\
\text { Conc. }\end{array}$ & $\begin{array}{l}\text { LCS } \\
\text { Result }\end{array}$ & $\begin{array}{c}\text { LCS } \\
\% \operatorname{ReC}\end{array}$ & $\begin{array}{l}\% \operatorname{Rec} \\
\text { Limits }\end{array}$ & Qualifiers \\
\hline Vinyl chloride & $u g /$. & & 10 & 9.6 & 96 & $59-124$ & \\
\hline Xylene (Total) & $u g / L$ & & 30 & 30.0 & 100 & $83-125$ & \\
\hline 1,2-Dichloroethane-d4 (S) & $\%$ & & & & 94 & $81-118$ & \\
\hline 4-Bromofluorobenzene (S) & $\%$ & & & & 103 & $85-119$ & \\
\hline Dibromofluoromethane (S) & $\%$ & & & & 100 & 85-114 & \\
\hline Toluene-d8 (S) & $\%$ & & & & 100. & $82-114$ & \\
\hline
\end{tabular}




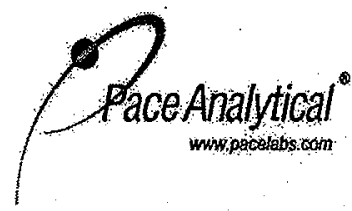

Pace Analytical Services, Inc. 9608 Loiret Blvd. Lenexa, KS 66219 (913)599-5665

\section{QUALIFIERS}

Project: Morrill-ANL

Pace Project No.: 6040555

\section{DEFINITIONS}

DF - Dilution Factor, if reported, represents the faclor applied to the reported data due to changes in sample preparation, dilution of the sample aliquot, or moisture content.

ND - Not Detected at or above adjusted reporting limit.

$\mathrm{J}$ - Estimated concentration above the adjusted method detection limit and below the adjusted reporting limit.

MDL - Adjusted Methód Detection Limit.

$S$ - Surrogate

1,2-Diphenylhydrazine (8270 listed analyte) decomposes to Azobenzene.

Consistent with EPA guidelines, unrounded data are displayed and have been used to calculate \% recovery and RPD values.

LCS(D) - Laboratory Control Sample (Duplicate)

MS(D) - Matrix Spike (Duplicate)

DUP - Sample Duplicate

RPD - Relative Percent Difference

Pace Analytical is NELAP accredited. Contact your Pace PM for the current list of accredited analytes.

\section{BATCH QUALIFIERS}

Batch: MSV/14700

[1] A matrix spike/matrix spike duplicate was not performed for this batch due to insufficient sample volume.

\section{ANALYTE QUALIFIERS}

B- $\quad$ Analyte detected in method blank but was not detected in the associated samples.

S6 Surrogate recovery outside control limits. Data accepted based on valid recovery of applicable surrogates (no analytes associated with this surrogate) 


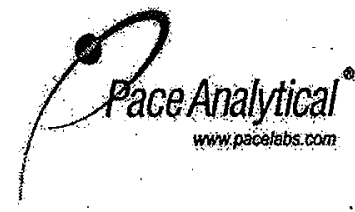

Pace Analytical Services, Inc. 9608 Loiret Blvd. Lenexa, KS 66219 (913)599-5665

QUALITY CONTROL DATA CROSS REFERENCE TABLE

$\begin{array}{ll}\text { Project: } & \text { Morrill-ANL } \\ \text { Pace Project No.: } & 6040555\end{array}$

\begin{tabular}{|c|c|c|c|c|c|}
\hline Lab ID & Sample ID & QC Batch Method & QC Batch & Analytical Method & $\begin{array}{l}\text { Analytical } \\
\text { Batch }\end{array}$ \\
\hline 6040555001 & PURGE WATER & EPA 504.1 & OEXT/11602 & EPA 504.1 & GCSV/5078 \\
\hline 6040555001 & PURGE WATER & EPA $5030 \mathrm{~B} / 8260$ & MSV/14706 & . & . \\
\hline 6040555002 & TRIP BLANK & EPA $5030 B / 8260$ & MSV/14706 & & \\
\hline
\end{tabular}




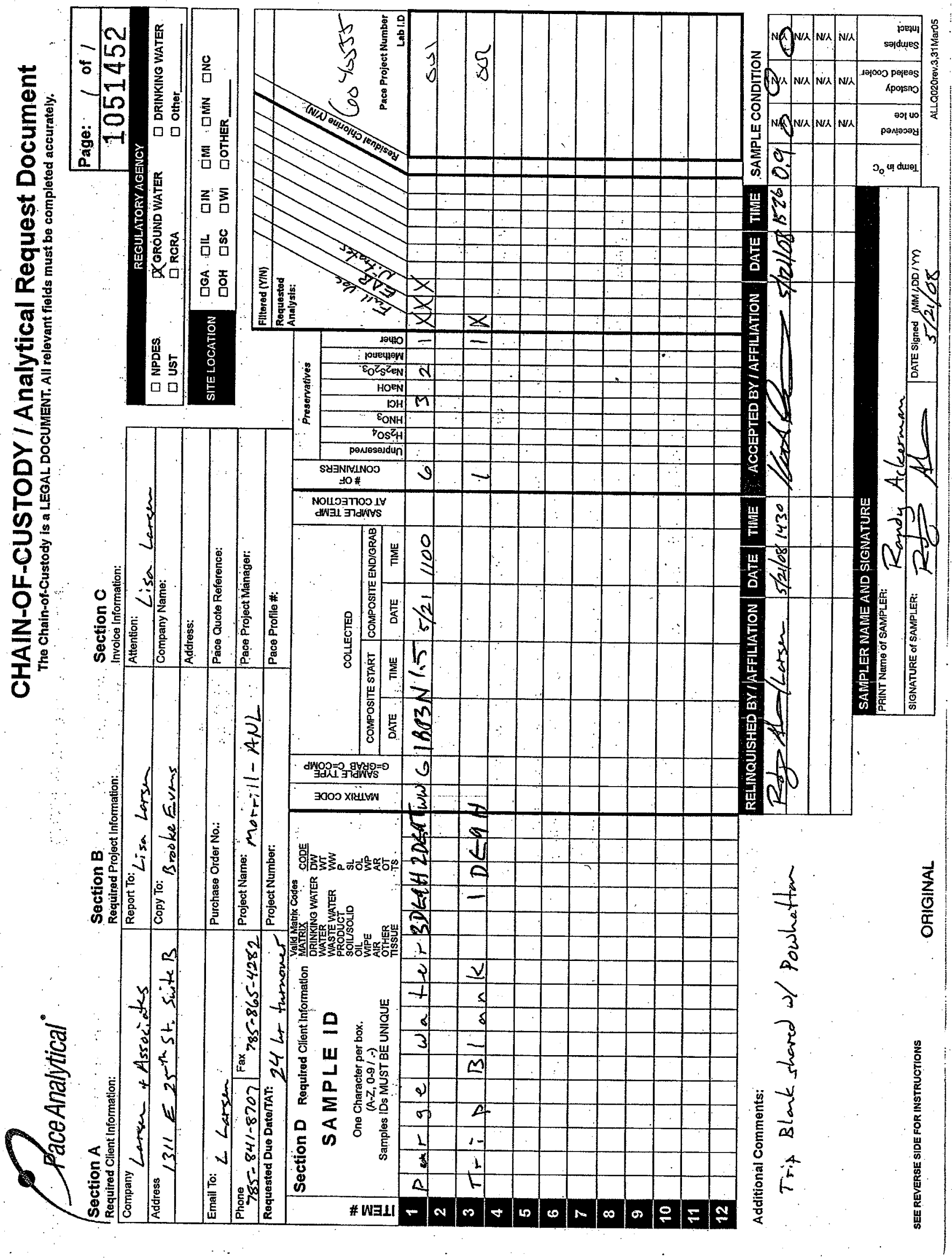


coutier: $\square$ Fed Ex $\square$ UPS $\square$ USPS $\square$ Crient $\square$ Commercial $\square$ Pace Other Tracking \#:

Custody Seal on CooleriBox Present: $\square$ yes in seals intact $\square$ yes 7 ro

Packing Material: $\square$ Bubbitie Wrop: 口Bubble Bags $\square$ None $\square$ Other

Chain of Custody Present Tres Q DNo DNeA 1.

Chain of Custody Fllled Out:

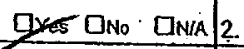

Chain of Custody Relinquished: Tyes ano DN/A 3.

Sampler Name \& Signature on COC:

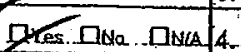

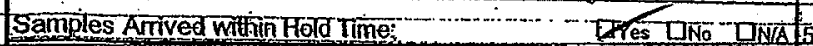

Short Hold Tine Anatysis ( 72 2hr): Dres DNo DNA E

Rush Tuth Around Fime Requested: CPres QNo CINA

Sufficient Volume:

Dres ano Diva 8.

Correct Containers Used:

Pace Containers Useds

Fres DNo. DNa 9 .

Containers intact:

Dres Dno DNA

Futered volume received for Dissolved tests:

EYes DNo DNHA 10.

Sample Labels match coc:

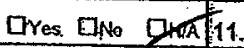

Dores Dino DNial12.

-Includes date/(inne/lB/Analysis Matrix.

Ail containers needfing. preservation fave been chocked:

Atl containers needing preservation are found to be in compllance with EPA reconmendation.

exéptions NOA. colliform, TOC, O\&G, WIORO (water)

$\omega T$

DTes, OTNO DNIA 13

Samples checked for dechlorination:

Pres ONo DNA

Headspace in VOA Vials $(>6 \mathrm{~mm})$ :

Trip Btark Present:

Trip Blank Custody Seals Eresent

Pace Trip Blank Lot \# (if paretiased) $031708-3$

thres Dino

Initial when

UYes DNo ZnN/A 14 .

toot \# of added

perservalive

Client Notification'Resolution:

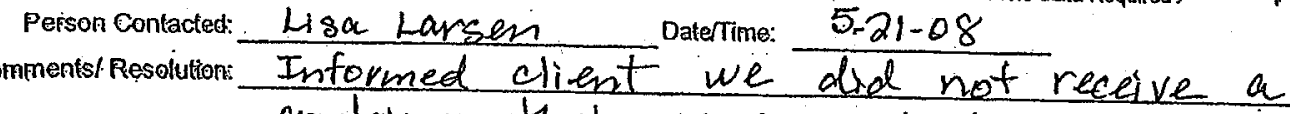

Qres Dowo DNA t5.

Dhes Uive DNA 16.

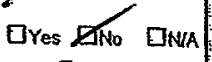

container that we can analyze the initrate os of

Project Manager Review: Sb \& $5,21.08$

Date:

Nofe: Whenever there is a discrepancy affeeling North Carolina compliance samples, a copy of this form will be sent to the North Carolina DEHNR

Certification Office (i.e out of hold; incorrecl preservative, out of temp, incorrect contalners) 


\section{Appendix D:}

\section{Data Summary for Verification VOCs Analyses by Envirosystems, Inc.}




\section{ENVIROSYSTEMS, INC.}

9200 Rumsey Road - Suite B102 - Columbia, Maryland 21045-1934

Phone (410) 964-0330 - Fax (410) 740-9306

Email: info@envsystems.com - Webpage: www.envsystems.com/envsys

Jorge S. Alvarado, Ph.D

Argonne National Laboratory

Environmental Research Division

Applied Geoscience and Environmental

Management Section

9700 South Cass Avenue, ER-203

Argonne, Illinois 60439

RE: Report\# 080170

Dear Jorge:

Enclosed are the results of analysis for the samples received on April 14, 2008 for volatile organics analysis by US EPA $8260 \mathrm{~B}$. The analytical results for this data package had been submitted byemail as requested by you.

Please do not hesitate to call me if you have any questions, comments, or require additional information.

Mohom Ole ox

Mohan Khare, Ph.D

President/CEO

MK/ncc 


\subsection{Narrative}




\section{Narrative}

This analytical data package contains the volatile organic analysis by USEPA SW-846 method $8260 \mathrm{~B}$ and CLP protocols. These samples. received April 16, 2008. The chain of Custody document in this report is in section 2.0, the analytical data summary is in section 3.1, sample data is in section 3.2, and standard raw QC data for BFB tuning, and MS/MSD in section 3.3 and 3.4 respectively. 


\subsection{Traffic Reports/ Chain of Custody Records}




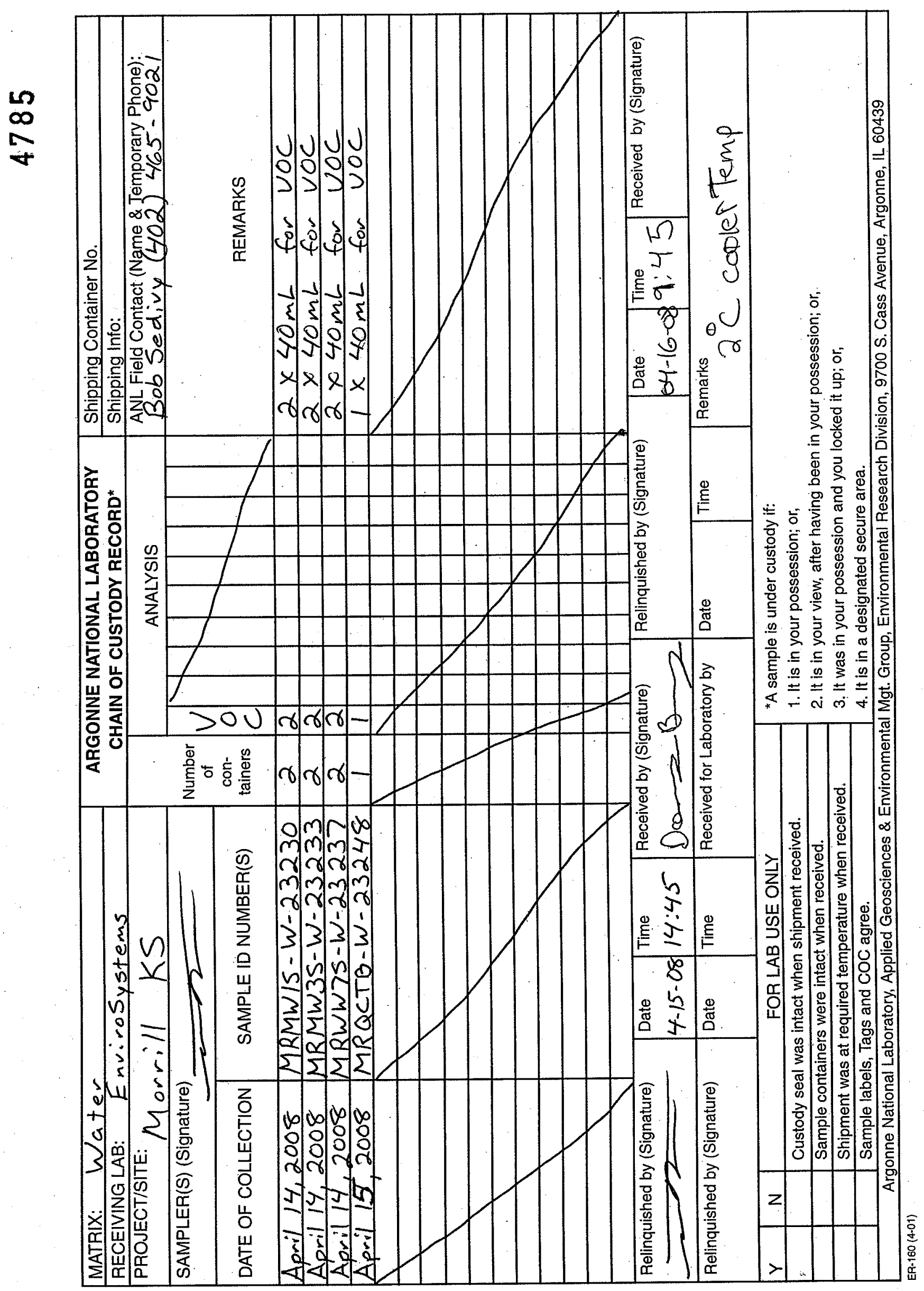




\subsection{VOA Data}


FORM 1

VOLATILE ORGANICS ANALYSIS DATA SHEET

Lab Name: ENVIROSYSTEMS, INC.

Lab Code: ENVSYS Case No.:

Matrix: (soil/water) WATER

Sample wt/vol: $\quad 5.000(\mathrm{~g} / \mathrm{mL}) \mathrm{ML}$

Level: (low/med) LOW

음 Moisture: not dec.

GC Column: RTX-624 ID: 0.18 (mm)

Soil Extract Volume:

(UL)

Contract: N/A
ARGONNE SAMPLE NO.

MRMWIS $-W-23230$

SAS NO.: N/A SDG No.: NA

Lab Sample ID: 0080405-01

Lab File ID: H001005

Date Received: 04/16/0.8 ...

Date Analyzed: 04/18/08

Dilution Factor: 1.0

Soil Aliquot Volume:

(uL)

CONCENTRATION UNITS:

CAS NO. COMPOUND. $\quad(u g / L$ or $\mathrm{ug} / \mathrm{Kg}$ ) UG/L Q

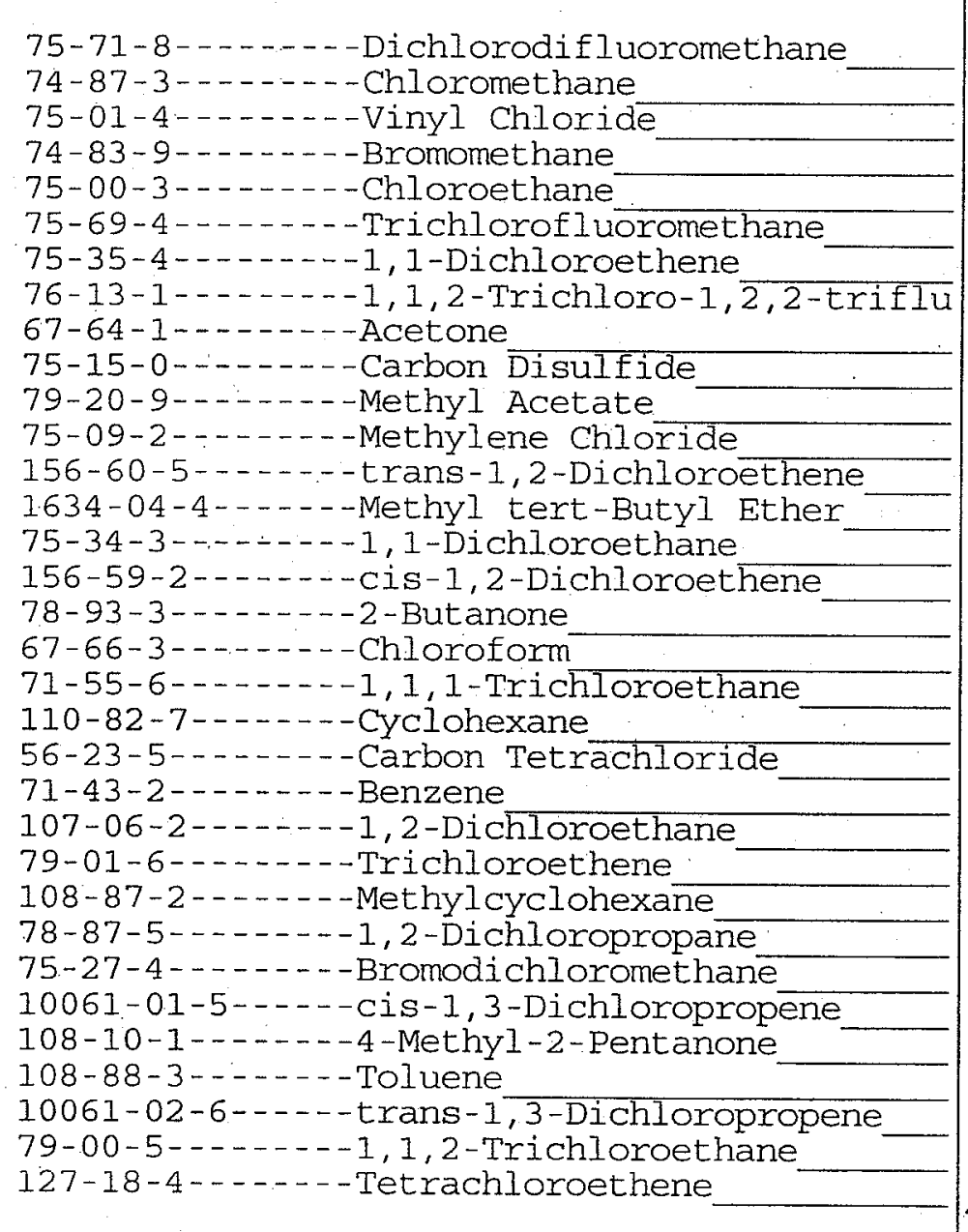

FORM I VOA

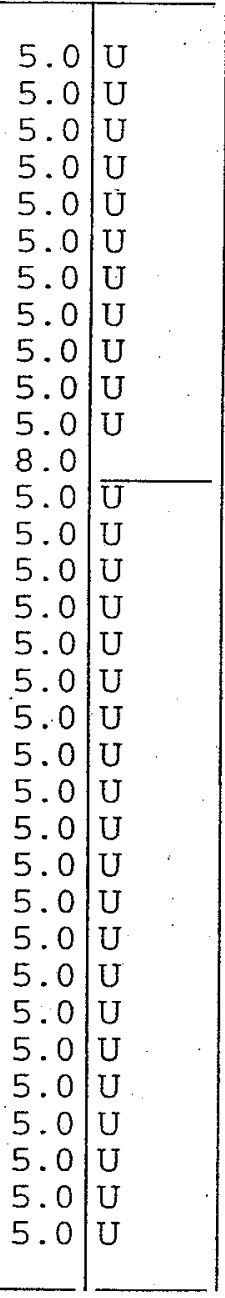

$5.0 \mathrm{U}$

$5.0 \mathrm{U}$

$5.0 \mathrm{U}$

政

$5.0 \mathrm{U}$

$5.0 \mathrm{U}$

$5.0 \mathrm{U}$

.

5 . 0

$5.0 \mathrm{U}$

$5.0 \mathrm{U}$

$5.0 \mathrm{U}$

$5.0 \mathrm{U}$

$5.0 \mathrm{U}$

$5.0 \mathrm{U}$

$5.0 \mathrm{U}$

$5.0 \mathrm{U}$

$5.0 \mathrm{U}$

$5.0 \mathrm{U}$

$5.0 \mathrm{U}$

$5.0 \mathrm{U}$

$5.0 \mathrm{U}$

$5.0 \mathrm{U}$

$5.0 \mathrm{U}$

$5.0 \mathrm{U}$

$5.0 \mathrm{U}$

$5.0 \mathrm{U}$

$5.0 \mathrm{U}$ 
FORM 1

VOLATILE ORGANICS ANALYSIS DATA SHEET

Lab Name: ENVIROSYSTEMS, INC.

Lab Code: ENVSYS Case No.:

Matrix: (soil/water) WATER

Sample wt/Vol: $\quad 5.000(\mathrm{~g} / \mathrm{mL}) \mathrm{ML}$

Lèvel: (low/med) ... Low

\% Moisture: not dec.

GC Column: RTX-624 ID: 0.18 (mm)

Soil Extract Volume:

(UL)
Contract: $N / A$

SAS NO.: N/A
ARGONNE SAMPLE NO.

MRMWIS -W- 23230

Lab Sample ID: 0080405-01

Lab File ID: H001005

Date Received: 04/16/08

Date Analyzed: 04/18/08

Dilution Factor: 1.0

Soil Aliquot Volume: (UI) CONCENTRATION UNITS: (ug/L or $u g / \mathrm{Kg}$ ) UG/L

Q

$5.0 \mathrm{U}$

$5.0 \mathrm{U}$

$5.0 \mathrm{U}$

$5.0 \mathrm{U}$

$5.0 \mathrm{U}$

$5.0 \mathrm{U}$

$5.0 \mathrm{U}$

$5.0 \mathrm{U}$

$5.0 \mathrm{U}$

$5.0 \mathrm{U}$

$5.0 \mathrm{U}$

$5.0 \mathrm{U}$

$5.0 \mathrm{U}$

$5.0 \mathrm{U}$

$5.0 \mathrm{U}$

$10 \mathrm{U}$

$5.0 \mathrm{U}$

$10 \mathrm{U}$

$10 \mathrm{U}$

$10 \mathrm{U}$

$10 \mathrm{U}$ 
FORM 1

VOLATILE ORGANICS ANALYSIS DATA SHEET

Lab Name: ENVIROSYSTEMS, INC.

Lab Code: ENVSYS Case No.:

Matrix: (soil/water) WATER

Sample wt/vol: $\quad 5.000(\mathrm{~g} / \mathrm{mL}) \mathrm{ML}$

Level: (low/med.) . LOW

\% Moisture: not dec.

GC Column: RTX-624 ID: 0.18 (mm)

Soil Extract Volume:

(UL)
Contract: $\mathrm{N} / \mathrm{A}$

SAS NO.: N/A
ARGONNE SAMPLE NO.

MRMW3S-W-23233

Lab Sample ID: 0080405-02

Lab File ID: H001006

Date Received: 04/16/08.

Date Analyzed: 04/18/08

Dilution Factor: 1.0

Soil Aliquot Volume:

(uL)

CAS NO.

COMPOUND

CONCENTRATION UNITS:

(ug/L or $\mathrm{ug} / \mathrm{Kg}$ ) UG/L

Q

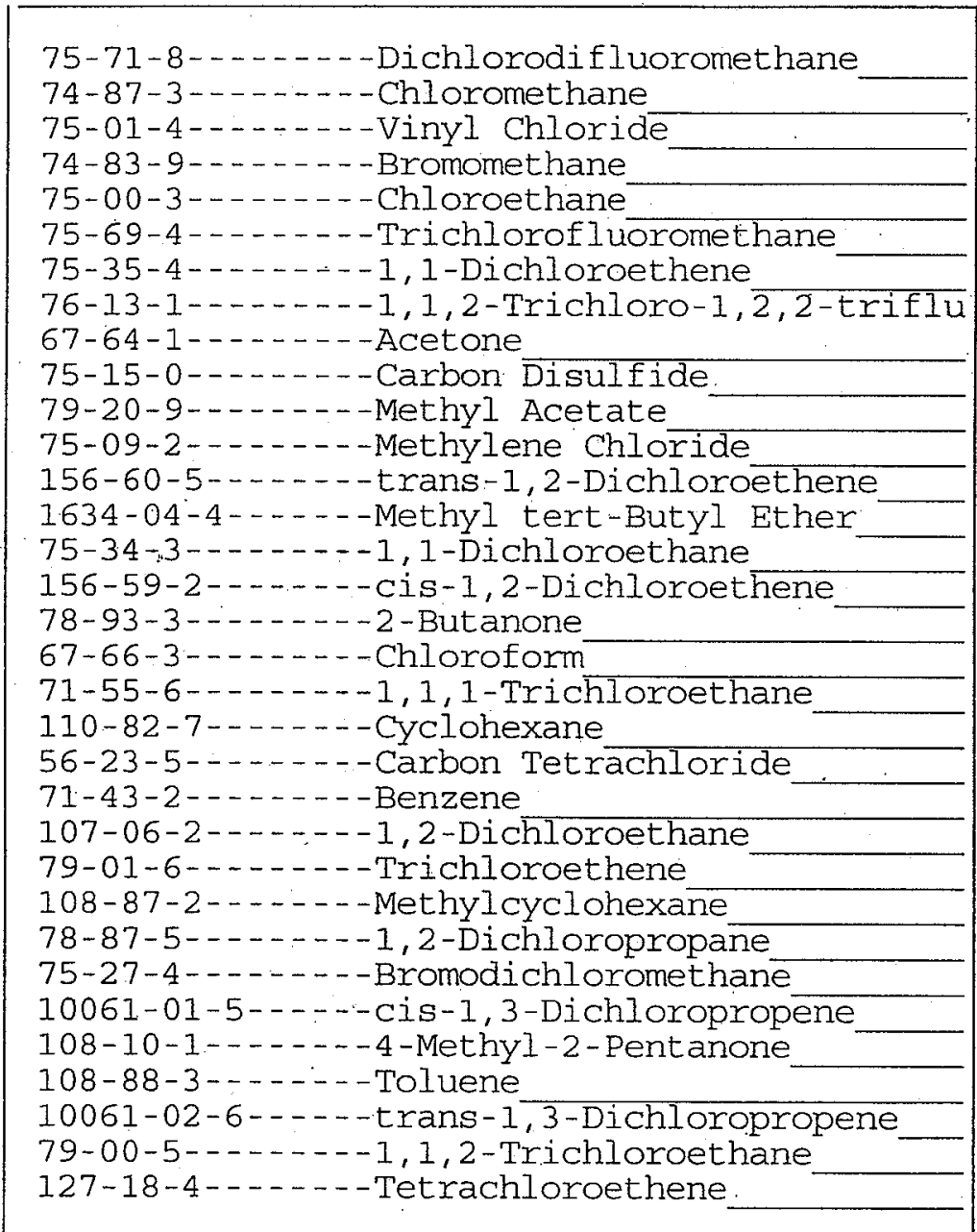

FORM I VOA

$5.0 \mathrm{U}$

$5.0 \mathrm{U}$

$5.0 \mathrm{U}$

$5.0 \mathrm{U}$

$5.0 \mathrm{U}$

$5.0 \mathrm{U}$

$5.0 \mathrm{U}$

$5.0 \mathrm{U}$

$5.0 \mathrm{U}$

$5.0 \mathrm{U}$

$5.0 \mathrm{U}$

7.5 .

$5.0 \overline{\mathrm{U}}$

$5.0 \mathrm{U}$

$5.0 \mathrm{U}$

$5.0 \mathrm{U}$

$5.0 \mathrm{U}$

$5.0 \mathrm{U}$

$5.0 \mathrm{U}$

$5.0 \mathrm{U}$

8.2

$5.0 \mathrm{U}$

$5.0 \mathrm{U}$

$5.0 \mathrm{U}$

$5.0 \mathrm{U}$

$5.0 \mathrm{U}$

$5.0 \mathrm{U}$

$5.0 \mathrm{U}$

$1.0 \mathrm{~J}$

$1.2 \mathrm{~J}$

$5.0 \mathrm{U}$

$5.0 \mathrm{U}$

$5.0 \mathrm{U}$ 
FORM 1

VOLATILE ORGANICS ANALYSIS DATA SHEET

Lab Name: ENVIROSYSTEMS, INC.

Contract: N/A

Lab Code: ENVSYS. Case No.:

SAS NO.: N/A
ARGONNE SAMPLE NO.

MRMW3S-W-23233
Matrix: (soil/water) WATER

Sample wt/vol: $\quad 5.000(\mathrm{~g} / \mathrm{mL}) \mathrm{ML}$

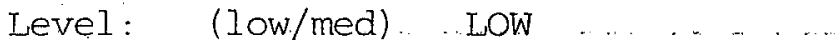

․oisture: not dec.

GC Column: RTX-624

ID $: 0.18$ (mm)

Soil Extract Volume:
Lab Sample ID: 0080405-02

Lab File ID: $\quad$ H001006

Date Received: 04/16/08

Date Analyzed: 04/18/08

Dilution Factor: 1.0

Soil Aliquot Volume: (UL)

CAS NO.

COMPOUND

CONCENTRATION UNITS:

(ug/L or ug/Kg) UG/L

Q

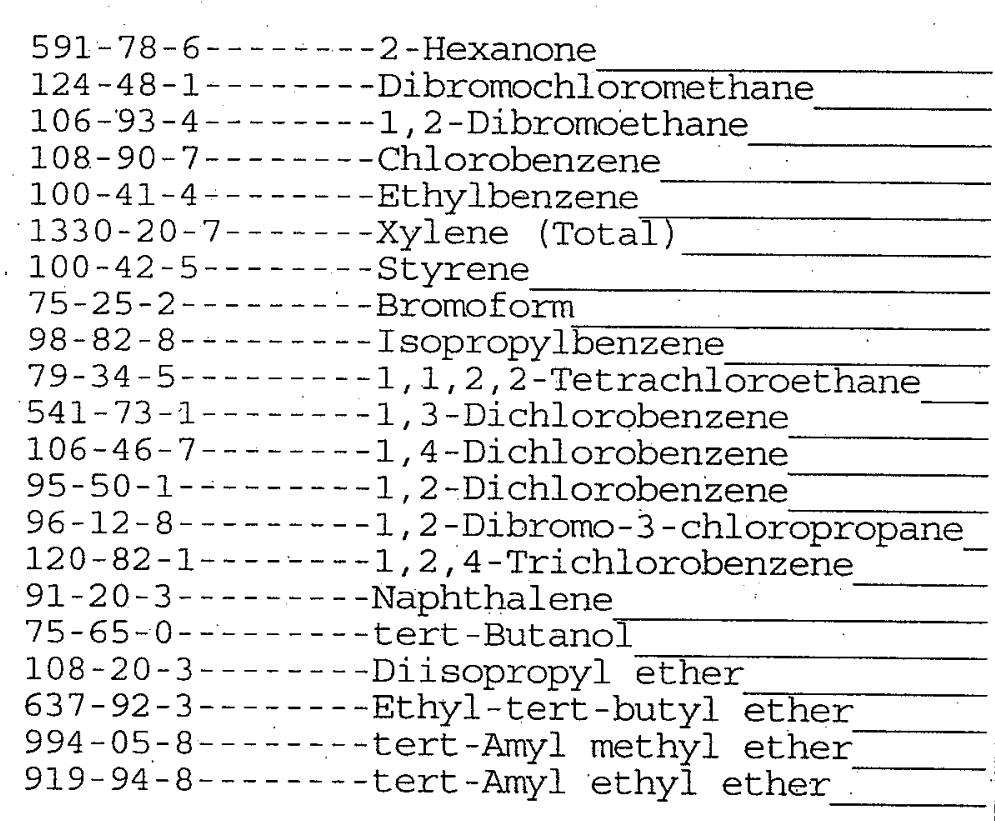

$5.0 \mathrm{U}$

$5.0 \mathrm{U}$

$5.0 \mathrm{U}$

$5.0 \mathrm{U}$

$5.0 \mathrm{U}$

$5.0 \mathrm{U}$

$5: 0 \mathrm{U}$

$5.0 \mathrm{U}$

$5.0 \mathrm{U}$

$5.0 \mathrm{U}$

$5.0 \mathrm{U}$

$5.0 \mathrm{U}$

$5.0 \mathrm{U}$

$5.0 \mathrm{U}$

$5.0 \mathrm{U}$

$10 \mathrm{U}$

$5.0 \mathrm{U}$

$10 \mathrm{U}$

$10 . \mathrm{U}$

$10 \mathrm{U}$

$10 \mathrm{U}$

FORM I VOA 
FORM 1

VOLATILE ORGANICS ANAIYSIS DATA SHEET

Lab Name: ENVIROSYSTEMS, INC.

Lab Code: ENVSYS Case No.:

Matrix: (soil/water) WATER

Sample wt/vol: $\quad 5.000(\mathrm{~g} / \mathrm{mL}) \mathrm{ML}$

Level :. (low/med) . LOW

$\because$ Moisture: not dec.

GC Column: RTX-624 ID: 0.18 (mm)

Soil Extract Volume:

(uL)
Contract: $N / A$

SAS NO.: N/A
ARGONNE SAMPLE NO.

MRMW7S-W-23237

CAS NO.

COMPOUND

Lab Sample ID: 0080405-03

Lab File ID: H001007

Date Received: 04/16/08

Date Analyzed: 04/18/08

Dilution Factor: 1.0

Soil Aliquot Volume:

(uL)

CONCENTRATION UNITS:

(ug/L or ug/Kg) UG/L

Q

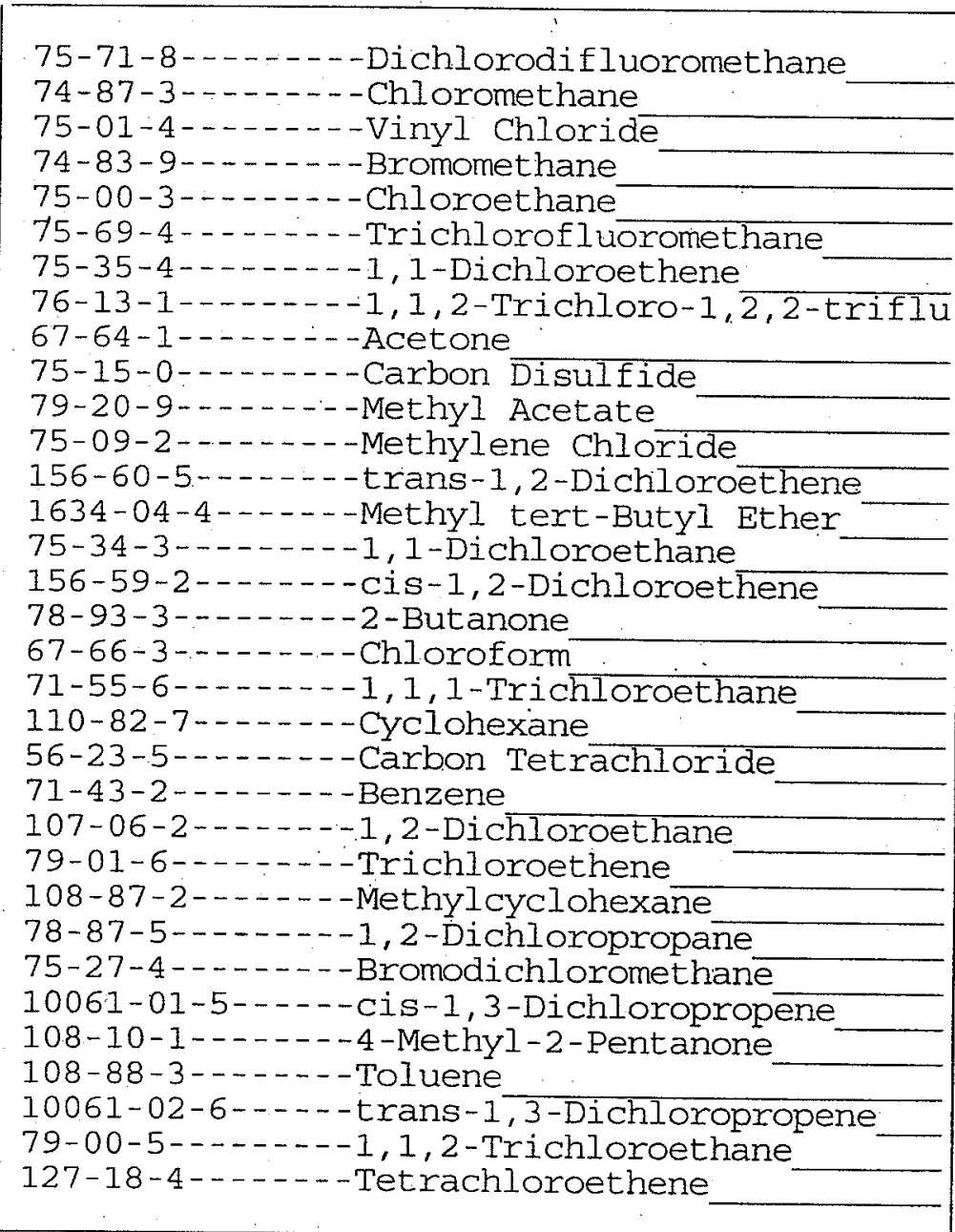

FORM I VOA

\begin{tabular}{l|l}
5.0 & $\mathrm{U}$ \\
5.0 & $\mathrm{U}$ \\
5.0 & $\mathrm{U}$ \\
5.0 & $\mathrm{U}$ \\
5.0 & $\mathrm{U}$ \\
5.0 & $\mathrm{U}$ \\
5.0 & $\mathrm{U}$ \\
5.0 & $\mathrm{U}$ \\
5.0 & $\mathrm{U}$ \\
5.0 & $\mathrm{U}$ \\
5.0 & $\mathrm{U}$ \\
8.9 & \\
5.0 & $\mathrm{U}$ \\
5.0 & $\mathrm{U}$ \\
5.0 & $\mathrm{U}$ \\
5.0 & $\mathrm{U}$ \\
5.0 & $\mathrm{U}$ \\
5.0 & $\mathrm{U}$ \\
5.0 & $\mathrm{U}$ \\
5.0 & $\mathrm{U}$ \\
10 & \\
5.0 & $\mathrm{U}$ \\
5.0 & $\mathrm{U}$ \\
5.0 & $\mathrm{U}$ \\
5.0 & $\mathrm{U}$ \\
5.0 & $\mathrm{U}$ \\
5.0 & $\mathrm{U}$ \\
5.0 & $\mathrm{U}$ \\
5.0 & $\mathrm{U}$ \\
1.4 & $\mathrm{~J}$ \\
5.0 & $\mathrm{U}$ \\
5.0 & $\mathrm{U}$ \\
5.0 & $\mathrm{U}$ \\
5.0 & \\
\hline
\end{tabular}


FORM 1

VOLATILE ORGANICS ANALYSIS DATA SHEET

Lab Name: ENVIROSYSTEMS, INC.

Lab Code: ENVSYS Case No.:

Matrix: (soil/water) WATER

Sample wt/vol: $\quad 5.000(\mathrm{~g} / \mathrm{mL}) \mathrm{ML}$

Level: (low/med) LOW

\% Moisture: not dec.

GC Column: RTX-624 ID: 0.18 (mm)

Soil Extract Volume:

(UL)

Contract: N/A

SAS NO.: N/A

SDG No.: NA

Lab Sample ID: 0080405-03

Lab File ID: H001007

Date Received: 04/16/08

Date Analyzed: 04/18/08

Dilution Factor: 1.0

Soil Aliquot Volume: (uL)

CAS NO.

COMPOUND

(ug/L or ug/Kg) UG/L

Q

$5: 0 \mathrm{U}$

5:0 U

$5.0 \mathrm{U}$

$5.0 \mathrm{U}$

$5.0 \mathrm{U}$

$5.0 \mathrm{U}$

$5.0 \mathrm{U}$

$5.0 \mathrm{U}$

$5.0 \mathrm{U}$

$5.0 \mathrm{U}$

$5.0 \mathrm{U}$

$5.0 \mathrm{U}$

$5.0 \mathrm{U}$

$5.0 \mathrm{U}$

$5.0 \mathrm{U}$

$10 \mathrm{U}$

$5.0 \mathrm{U}$

$10 \mathrm{U}$

$10 \mathrm{U}$

$10 \mathrm{U}$

$10 \mathrm{U}$

FORM I VOA 
FORM 1

VOLATILE ORGANICS ANALYSIS DATA SHEET

Lab Name: ENVIROSYSTEMS, INC.

Lab Code: ENVSYS Case No.:

Matrix: (soil/water) WATER

Sample wt/vol: $\quad 5.000(\mathrm{~g} / \mathrm{mL}) \mathrm{ML}$

Level :... (low/med).. LOW

․ Moisture: not dec.

GC Column: RTX-624 ID: 0.18 (mm)

Soil Extract Volume:

(uL)
ARGONNE SAMPLE NO.

MRQCTB-W-23248
Contract: N/A

SAS NO.: N/A SDG No.: NA
Lab Sample ID: 0080405-04

Lab File ID: H001010.

Date Received: 04/16/08

Date Analyzed: 04/18/08

Dilution Factor: 1.0

Soil Aliquot Volume: (uL)

\begin{tabular}{|c|c|c|}
\hline 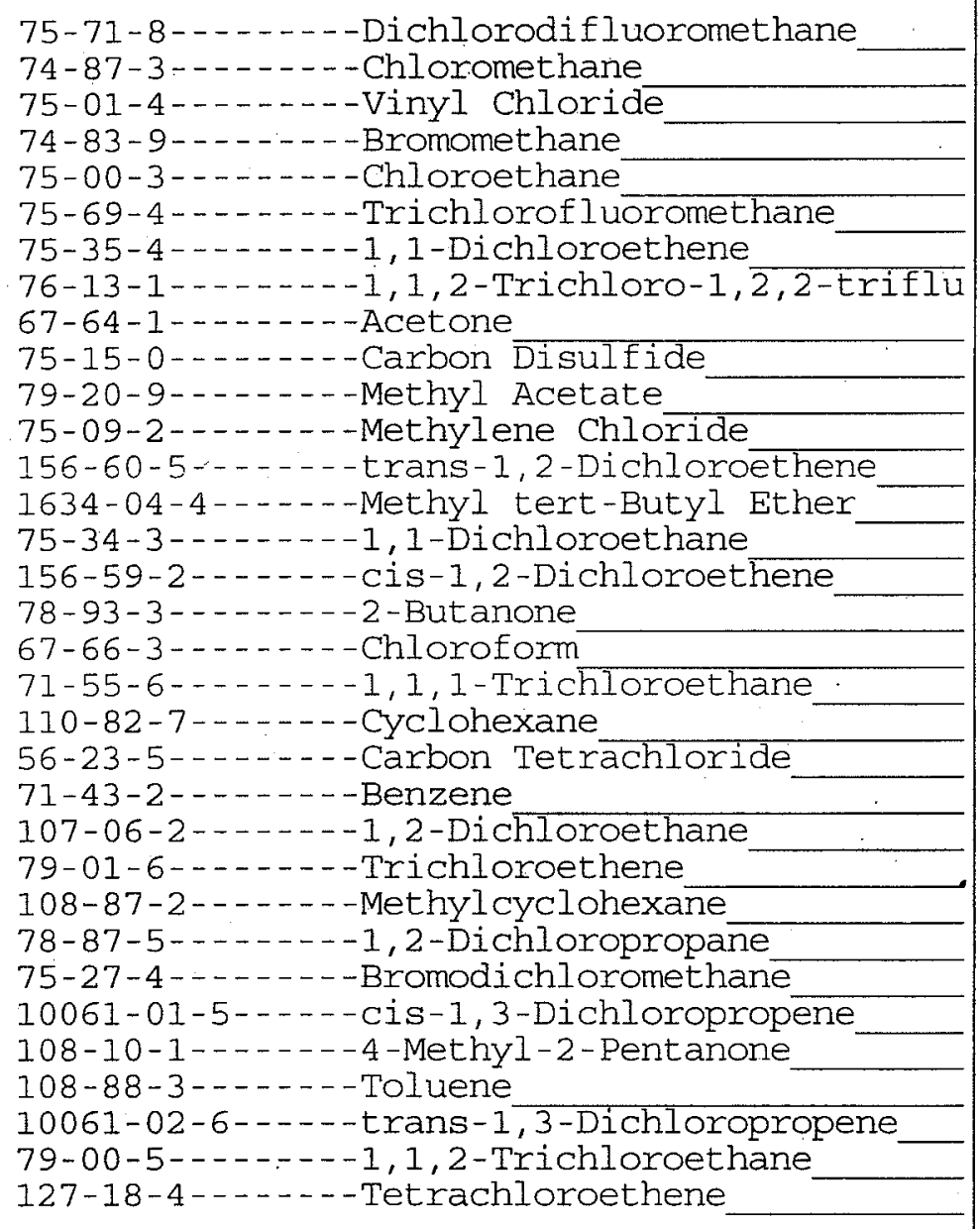 & $\begin{array}{l}5.0 \\
5.0 \\
5.0 \\
5.0 \\
5.0 \\
5.0 \\
5.0 \\
5.0 \\
5.0 \\
5.0 \\
5.0 \\
6.5 \\
5.0 \\
5.0 \\
5.0 \\
5.0 \\
5.0 \\
5.0 \\
5.0 \\
5.0 \\
5.0 \\
5.0 \\
5.0 \\
5.0 \\
5.0 \\
5.0 \\
5.0 \\
5.0 \\
5.0 \\
5.0 \\
5.0 \\
5.0 \\
5.0\end{array}$ & $\begin{array}{l}U \\
U \\
U \\
U \\
U \\
U \\
U \\
U \\
U \\
U \\
U \\
U \\
U \\
U \\
U \\
U \\
U \\
U \\
U \\
U \\
U \\
U \\
U \\
U \\
U \\
U \\
U \\
U \\
U \\
U \\
U \\
U \\
U \\
U \\
U\end{array}$ \\
\hline
\end{tabular}

FORM I VOA 
FORM 1

VOLATILE ORGANICS ANALYSIS DATA SHEET

Lab Name: ENVIROSYSTEMS, INC.

Lab Code: ENVSYS Case No.:

Matrix: (soil/water) WATER

Sample wt/vol: $\quad 5.000(\mathrm{~g} / \mathrm{mL}) \mathrm{ML}$

Level.. (low/med). LOW

\% Moisture: not dec.

GC Column: RTX-624 ID: 0.18 (mm)

Soil Extract Volume:

(uL)

Contract: $\mathrm{N} / \mathrm{A}$

SAS NO.: N/A

Lab Sample ID: 0080405-04

Lab File ID: H001010

Date Rece.ived: 04/16/08

Date Analyzed: 04/18/08

Dilution Factor: 1.0

Soil. Aliquot Volume: (uL)

CAS NO. COMPOUND

TRATION UNITS:

(ug/L or ug/Kg) UG/L

Q

$5.0 \mathrm{U}$

$5.0 \mathrm{U}$

$5.0 \mathrm{U}$

$5.0 \mathrm{U}$

$5.0 \mathrm{U}$

$5.0 \mathrm{U}$

$5.0 \mathrm{U}$

$5.0 \mathrm{U}$

$5.0 \mathrm{U}$

$5.0 \cdot \mathrm{U}$

$5.0 \mathrm{U}$

$5.0 \mathrm{U}$

$5.0 \mathrm{U}$

$5.0 \mathrm{U}$

$5.0 \mathrm{U}$

$10 \mathrm{U}$

$5.0 \mathrm{U}$

$10 \mathrm{U}$

$10 \mathrm{U}$

$10 \mathrm{U}$

$10 / \mathrm{U}$ 
FORM 1

VOLATILE ORGANICS ANALYSIS DATA SHEET

Lab Name: ENVIROSYSTEMS, INC.

Lab Code: ENVSYS Case No.:

Matrix: (soil/water) WATER

Sample wt/vol: $\quad 5.000(\mathrm{~g} / \mathrm{mL}) \mathrm{ML}$

Level: (low/med) LOW

\% Moisture: not dec.

GC Column: RTX-624 ID: 0.18 (mm)

Soil Extract Volume:
Contract: $\mathrm{N} / \mathrm{A}$

SAS NO.: N/A
ARGONNE SAMPLE NO.

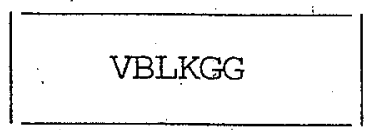

SDG No.: NA
Lab Sample ID: 0000848-BLK1

Lab File ID: ' H001003

Daté Received: 04/16/08

Date Analyzed: 04/18/08

Dilution Factor: 1.0

Soil Aliquot Volume: (uL)

CAS NO.

COMPOUND

CONCENTRATION UNITS:

(ug/L or ug/Kg) UG/L

Q

$5.0 \mathrm{U}$

$5.0 \mathrm{U}$

$5.0 \mathrm{U}$

$5.0 \mathrm{U}$

$5.0 \mathrm{U}$

$5.0 \mathrm{U}$

$5.0 \mathrm{U}$

$5.0 \mathrm{U}$

$5.0 \mathrm{U}$

$5.0 \mathrm{U}$

$5.0 \mathrm{U}$

9.4

$5.0 \overline{\mathrm{U}}$

$5.0 \mathrm{U}$

$5.0 \mathrm{U}$

$5.0 \mathrm{U}$

$5.0 \mathrm{U}$

$5.0 \mathrm{U}$

$5.0 \mathrm{U}$

$5.0 \mathrm{U}$

$5.0 \mathrm{U}$

$5.0 \mathrm{U}$

$5.0 \mathrm{U}$

$5.0 \mathrm{U}$

$5.0 \mathrm{U}$

$5.0 \mathrm{U}$

$5.0 \mathrm{U}$

$5.0 \mathrm{U}$

$5.0 \mathrm{U}$

$5.0 \mathrm{U}$

$5.0 \mathrm{U}$

$5.0 \mathrm{U}$

$5.0 \mathrm{U}$ 
FORM 1

VOLATILE ORGANICS ANALYSIS DATA SHEET

Lab Name: ENVIROSYSTEMS, INC.

Lab Code: ENVSYS Case No: :

Matrix: (soil/water) WATER

Sample wt/vol: $\quad 5.000(\mathrm{~g} / \mathrm{mL}) \mathrm{ML}$

Level: (low/med). LOW

Contract: N/A

SAS NO.: N/A

Lab Sample ID : 0000848-BLKI

Lab File ID: H001003

Date Received: 04/16/08

\% Moisture: not dec.

GC Column: RTX-624 ID: 0.18 (mm)

(uL)

Soil Extract Volume:

Date Analyzed: 04/18/08

Dilution Factor: 1.0

Soil Aliquot Volume: (uL)

CAS NO.

COMPOUND

(ug/L or $\mathrm{ug} / \mathrm{Kg}$ ) UG/L

Q

$5.0 \mathrm{U}$

$5.0 \mathrm{U}$

$5.0 \mathrm{U}$

$5.0 \mathrm{U}$

$5.0 \mathrm{U}$

$5.0 \mathrm{U}$

$5.0 \mathrm{U}$

$5.0 \mathrm{U}$

$5.0 \mathrm{U}$

$5.0 \mathrm{U}$

$5.0 \mathrm{U}$

$5.0 \mathrm{U}$

$5.0 \mathrm{U}$

$5.0 \mathrm{U}$

$5.0 \mathrm{U}$

$10 \mathrm{U}$

$5.0 \mathrm{U}$

$10 \mathrm{U}$

$10 \mathrm{U}$

$10 \mathrm{U}$

$10 \mathrm{U}$ 


\section{ENVIROSYSTEMS, INC.}

9200 Rumsey Road • Suite B102 • Columbia, Maryland 21045-1934

Phone (410) 964-0330 - Fax (410) 740-9306

Email: info@envsystems.com -Webpage: www.envsystems.com/envsys

Jorge S. Alvarado, Ph.D

Argonne National Laboratory

Environmental Research Division

Applied Geoscience and Environmental

Management Section

9700 South Cass Avenue, ER-203

Argonne, Illinois 60439

RE: Report\# 080186

Dear Jorge:

Enclosed are the results of analysis for the samples received on April 23, 2008 for volatile organics analysis by US EPA $8260 \mathrm{~B}$. The analytical results for this data package had been submitted as email as requested by you.

Please do not hesitate to call me if you have any questions, comments, or require additional information.

Sincerely,<smiles>C=C(C)OC(=O)O</smiles>

Mohan Khare, Ph.D

President/CEO

$\mathrm{MK} / \mathrm{ncc}$ 
1. Narrative 


\section{Narrative}

This analytical data package contains the volatile organic analysis by USEPA SW-846 method $8260 \mathrm{~B}$ and CLP protocols for samples received April 23, 2008.

The Chain of Custody document for this report is in section 2, the analytical data summary, sample data, and standards data is in section 3 . 
2. Traffic Reports/ Chain of Custody Records 


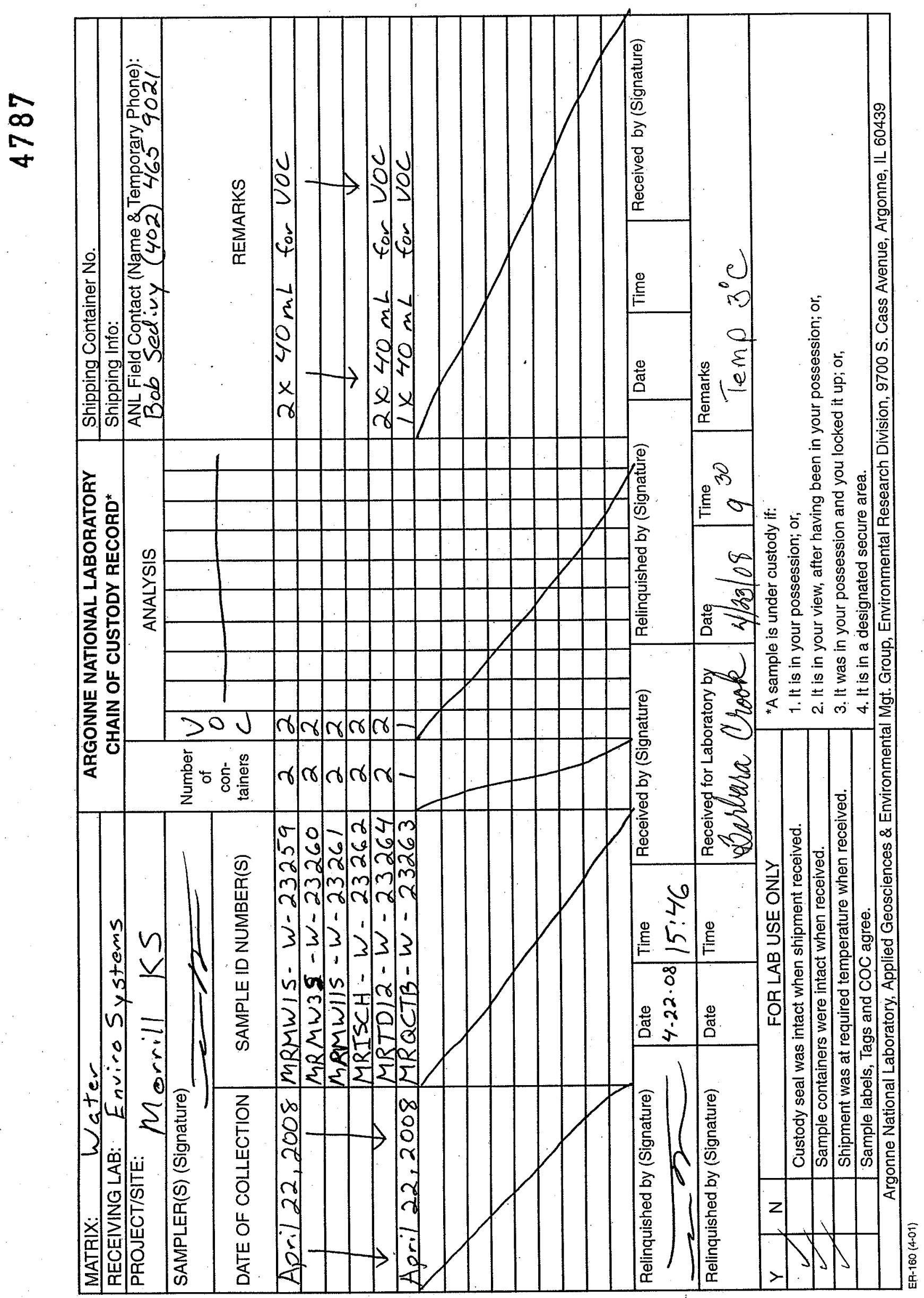


3. VOA Data 
FORM 1

VOLATILE ORGANICS ANALYSIS DATA SHEET

Lab Name: ENVIROSYSTEMS, INC.

Lab Code: ENVSYS Case No.:

Matrix: (soil/water) WATER

Sample wt/vol: $\quad 5.000(\mathrm{~g} / \mathrm{mL}) \mathrm{ML}$

Level: (low/med) LOW

\% Moisture: not dec.

GC Column: RTX-624 ID: 0.18 (mm)

Soil Extract Volume: (UL)

Contract: N/A

SAS NO.: N/A
ARGONNE SAMPLE NO.

MRMWIS-W-23259

Lab Sample ID: 0080410-01

Lab File ID: $\quad$ H001092

Date Received: 04/23/08

Date Analyzed: 04/23/08

Dilution Factor: 1.0

Soil Aliquot Volume: (uL)

CONCENTRATION UNITS:

CAS NO.

COMPOUND

(ug/L or ug/Kg) UG/L

\section{Q}

$5.0 \mathrm{U}$

$5.0 \mathrm{U}$

$5.0 \mathrm{U}$

$5.0 \mathrm{U}$

$5.0 \mathrm{U}$

$5.0 \mathrm{U}$

$5.0 \mathrm{U}$

$5.0 \mathrm{U}$

$5.0 \mathrm{U}$

5.0. U

$5.0 \mathrm{U}$

$3.0 \mathrm{~J}$

$5.0 \mathrm{U}$

$5.0 \mathrm{U}$

$5.0 \mathrm{U}$

$5.0 \mathrm{U}$

$5.0 \mathrm{U}$

$5.0 \mathrm{U}$

$5.0 \mathrm{U}$

$5.0 \mathrm{U}$

$5.0 \mathrm{U}$

$5.0 \mathrm{U}$

$5.0 \mathrm{U}$

$5.0 \mathrm{U}$

$5.0 \mathrm{U}$

$5.0 \mathrm{U}$

$5.0 \mathrm{U}$

$5.0 \mathrm{U}$

$5.0 \mathrm{U}$

$1.0 \mathrm{~J}$

$5.0 \mathrm{U}$

$5.0 \mathrm{U}$

$5.0 \mathrm{U}$ 
FORM 1

VOLATILE ORGANICS ANALYSIS DATA SHEET

Lab Name: ENVIROSYSTEMS, INC.

Case No. :

Matrix: (soil/water) WATER

Sample wt/vol: $\quad 5.000(\mathrm{~g} / \mathrm{mL}) \mathrm{ML}$

Level: (low/med) LoW

\% Moisture: not dec.

GC Column: RTX-624 ID: 0.18 (mm)

Soil Extract Volume:

(uL)
Contract: $\mathrm{N} / \mathrm{A}$

SAS NO.: N/A
ARGONNE SAMPLE NO.

MRMWIS-W-23259
SDG NO.: NA
Lab Sample ID: 0080410-01

Lab File ID:' H001092

Date Received: 04/23/08

Date Analyzed: 04/23/08

Dilution Factor: 1.0

Soil Aliquot Volume: (UL)

CAS NO.

COMPOUND

CONCENTRATION UNITS:

(ug/L or $\mathrm{ug} / \mathrm{Kg}$ ) UG/L

$5.0 \mathrm{U}$

$5.0 \mathrm{U}$

$5.0 \mathrm{U}$

$5.0 \mathrm{U}$

$5.0 \mathrm{U}$

$5.0 \mathrm{U}$

5.0 U

$5.0 \mathrm{U}$

$5.0 \mathrm{U}$

$5.0 \mathrm{U}$

$5.0 \mathrm{U}$

$5.0 \mathrm{U}$

$5.0 \mathrm{U}$

$5.0 \mathrm{U}$

$5.0 \mathrm{U}$

$10 \mathrm{U}$

$5.0 \mathrm{U}$

$10 \mathrm{U}$

$10 \mathrm{U}$

$10 \mathrm{U}$

$10 \mathrm{U}$ 
FORM 1

VOLATILE ORGANICS ANALYSIS DATA SHEET

Lab Name: ENVIROSYSTEMS, INC.

Lab Code: ENVSYS Case No.:

Matrix: (soil/water) WATER

Sample wt/vol: $\quad 5.000(\mathrm{~g} / \mathrm{mL})$ ML

Level: (low/med) LOW

\% Moisture: not dec.

GC Column: RTX-624 ID: 0.18 (mm)

Soil Extract Volume:

(uL)
Contract: $\mathrm{N} / \mathrm{A}$

SAS NO.: N/A
ARGONNE SAMPLE NO.

MRMWIS-W-23260

Lab Sample ID: 0080410-02

Lab File ID: H001093

Date Received: 04/23/08

Date Analyzed: 04/23/08

Dilution Factor: 1.0

Soil Aliquot volume:

(uL)

CONCENTRATION UNITS:

CAS NO.

COMPOUND

(ug/L or $\mathrm{ug} / \mathrm{Kg}$ ) UG/L

Q

$5.0 \mathrm{U}$

$5.0 \mathrm{U}$

$5.0 \mathrm{U}$

$5.0 \mathrm{U}$

$5.0 \mathrm{U}$

$5.0 \mathrm{U}$

$5.0 \mathrm{U}$

$5.0 \mathrm{U}$

$5.0 \mathrm{U}$

$5.0 \mathrm{U}$

$5.0 \mathrm{U}$

$3.1 \mathrm{~J}$

$5.0 \mathrm{U}$

$5.0 \mathrm{U}$

$5.0 \mathrm{U}$

$5.0 \mathrm{U}$

$5.0 \mathrm{U}$

$5.0 \mathrm{U}$

$5.0 \mathrm{U}$

$5.0 \mathrm{U}$

$5.0 \mathrm{U}$

$5.0 \mathrm{U}$

$5.0 \mathrm{U}$

$5.0 \mathrm{U}$

$5.0 \mathrm{U}$

$5.0 \mathrm{U}$

$5.0 \mathrm{U}$

$5.0 \mathrm{U}$

$5.0 \mathrm{U}$

$5.0 \mathrm{U}$

$5.0 \mathrm{U}$

$5.0 \mathrm{U}$

$5.0 \mathrm{U}$ 
FORM 1 VOLATILE ORGANICS ANALYSIS DATA SHEET

Lab Name: ENVIROSYSTEMS, INC. Lab Code: ENVSYS Case No.:

Matrix: (soil/water) WATER

Sample wt/vol:

$5.000(\mathrm{~g} / \mathrm{mL}) \mathrm{ML}$

Level: (low/med) LOW

\% Moisture: not dec.

GC Column: RTX-624 ID: 0.18 (mm)

Soil Extract Volume: (UL)
Contract: N/A

SAS NO.: N/A
ARGONNE SAMPLE NO.

MRMWIS -W-23260

Lab Sample ID: 0080410-02

Lab File ID: H001093

Date Received: 04/23/08

Date Analyzed: 04/23/08

Dilution Factor: 1.0

Soil Aliquot Volume:

CONCENTRATION UNITS:

CAS NO.

COMPOUND

(ug/I or $\mathrm{ug} / \mathrm{Kg}$ ) UG/L

Q

591-78-6-------2-Hexanone

124-48-1-...--Dibromochloromethane

106-93-4------1,2-Dibromoethane

108-90-7-....-Chlorobenzene

100-41-4_.....-Ethylbenzene

1330-20-7--.--Xylene (Total)

100-42-5--.--- Styrene

75-25-2-...-- Bromoform

98-82-8------- Isopropyl benzene

79-34-5 -....-1, 1,2, 2-Tetrachloroethane

541-73-1-n-.-1,3-Dichlorobenzene

106-46-7-----1, 4-Dichlorobenzene

95-50-1-------1, 2-Dichlorobenzene

96-12-8-.....- - 1, 2-Dibromo-3-chloropropane

120-82-1------1, 2, 4-Trichlorobenzene

91-20-3-...-.-Naphthalene

75-65-0-....-tert-Butanol

108-20-3--.--Dilsopropyl ether

637-92-3-..---Ethyl-tert-butyl ether

994-05-8-...-.tert-Amyl methyl ether

919-94-8-.....tert-Amyl ethyl ether
$5.0 \mathrm{U}$

$5.0 \mathrm{U}$

$5.0 \mathrm{U}$

$5.0 \mathrm{U}$

$5.0 \mathrm{U}$

$5.0 \mathrm{U}$

$5.0 \mathrm{U}$

$5.0 \mathrm{U}$

$5.0 \mathrm{U}$

$5.0 \mathrm{U}$

$5.0 \mathrm{U}$

$5.0 \mathrm{U}$

$5.0 \mathrm{U}$

$5.0 \mathrm{U}$

$5.0 \mathrm{U}$

$10 \mathrm{U}$

$5.0 \mathrm{U}$

$10 \mathrm{U}$

$10 \mathrm{U}$

$10 \mathrm{U}$

$10 \mathrm{U}$ 
FORM 1

VOLATILE ORGANICS ANALYSIS DATA SHEET

Lab Name: ENVIROSYSTEMS, INC.

Lab Code: ENVSYS Case No.:

Matrix: (soil/water) WATER

Sample wt/vol: $\quad 5.000(\mathrm{~g} / \mathrm{mL})$ ML

Level: (low/med) LOW

\% Moisture: not dec.

GC Column: RTX-624 ID: 0.18 (mm)

Soil Extract Volume:

(uL)
Contract: $N / A$

SAS NO.: N/A
ARGONNE SAMPLE NO.

MRMWIS-W-23261

Lab Sample ID: 0080410-03

Lab File ID: H001094

Date Received: 04/23/08

Date Analyzed: 04/23/08

Dilution Factor: 1.0

Soil Aliquot Volume: (uL)

CONCENTRATION UNITS:

CAS NO. COMPOUND $(u g / L$ or $\cdot u g / K g) ~ U G / L$

$Q$

$5.0 \mathrm{U}$

$5.0 \mathrm{U}$

$5.0 \mathrm{U}$

$5.0 \mathrm{U}$

$5.0 \mathrm{U}$

$5.0 \mathrm{U}$

$5.0 \mathrm{U}$

$5.0 \mathrm{U}$

$5.0 \mathrm{U}$

$5.0 \mathrm{U}$

$5.0 \mathrm{U}$

$3.5 \mathrm{~J}$

$5.0 \mathrm{U}$

$5.0 \mathrm{U}$

$5.0 \mathrm{U}$

$5.0 \mathrm{U}$

$5.0 \mathrm{U}$

$1.1 \mathrm{~J}$

$5.0 \mathrm{U}$

$5.0 \mathrm{U}$

46

$5.0 \mathrm{U}$

$5.0 \mathrm{U}$

$5.0 \mathrm{U}$

$5.0 \mathrm{U}$

$5.0 \mathrm{U}$

$5.0 \mathrm{U}$

$5.0 \mathrm{U}$

$5.0 \mathrm{U}$

$5.0 \mathrm{U}$

$5.0 \mathrm{U}$

$5.0 \mathrm{U}$

$5.0 \mathrm{U}$ 
FORM 1

VOLATILE ORGANICS ANALYSIS DATA SHEET

Lab Name: ENVIROSYSTEMS, INC.

Lab Code: ENVSYS Case No.:

Matrix: (soil/water) WATER

Sample wt/vol: $\quad 5.000(\mathrm{~g} / \mathrm{mL})$ ML

Level: (low/med) LOW

\% Moisture: not dec.

GC Column: RTX-624 ID: 0.18 (mm)

Soil Extract Volume:

(uL)
Contract: $\mathrm{N} / \mathrm{A}$

SAS NO.: N/A
ARGONNE SAMPLE NO.

MRMWIS-W-23261

Lab Sample ID: 0080410-03

Lab File ID: H001094

Date Received: 04/23/08

Date Analyzed: 04/23/08

Dilution Factor: 1.0

Soil Aliquot Volume: (UI)

CONCENTRATION UNITS:

CAS NO. COMPOUNDD $(\mathrm{ug} / \mathrm{L}$ or $\mathrm{ug} / \mathrm{Kg}) \mathrm{UG} / \mathrm{L} \quad \mathrm{Q}$

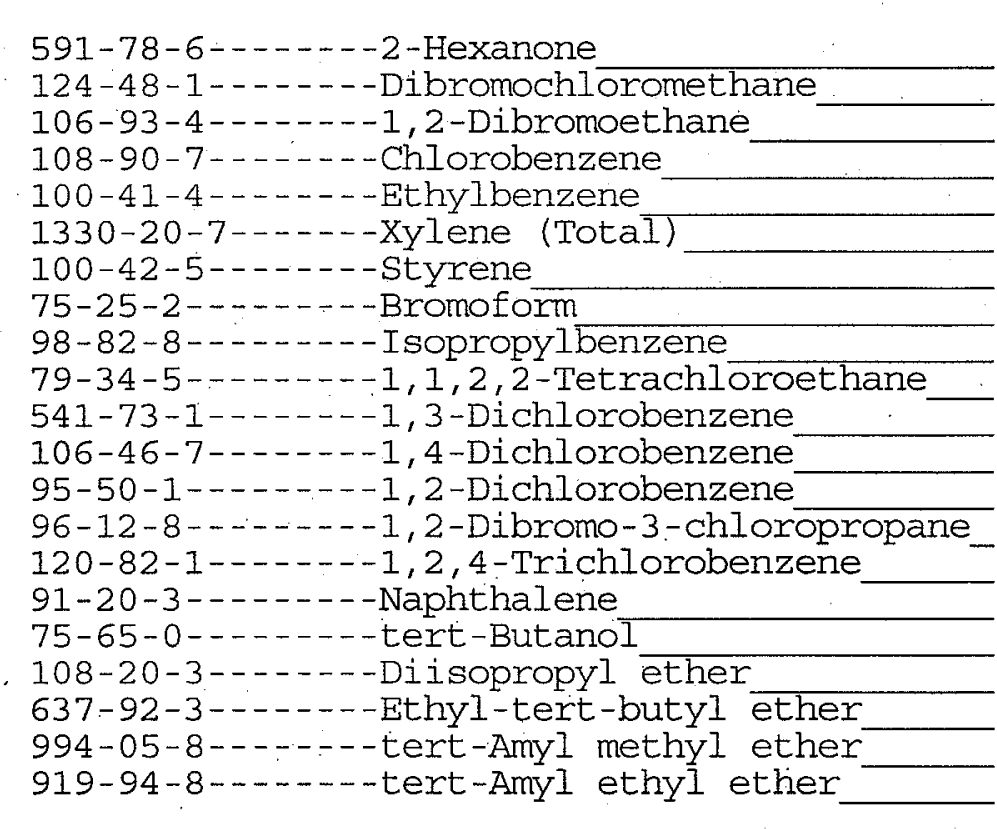

$5.0 \mathrm{U}$

$5.0 \mathrm{U}$

$5.0 \mathrm{U}$

$5.0 \mathrm{U}$

$5.0 \mathrm{U}$

$5.0 \mathrm{U}$

$5.0 \mathrm{U}$

$5.0 \mathrm{U}$

$5.0 \mathrm{U}$

$5.0 \mathrm{U}$

$5.0 \mathrm{U}$

$5.0 \mathrm{U}$

$5.0 \mathrm{U}$

$5.0 \mathrm{U}$

$5.0 \mathrm{U}$

$10 \mathrm{U}$

$5.0 \mathrm{U}$

$10 \mathrm{U}$

$10 \mathrm{U}$

$10 \mathrm{U}$

$10 \mathrm{U}$ 
FORM 1

VOLATILE ORGANICS ANALYSIS DATA SHEET

Lab Name: ENVIROSYSTEMS, INC.

Lab Code: ENVSYS Case No.:

Matrix: (soil/water) WATER

Sample wt/vol:

$5.000(\mathrm{~g} / \mathrm{mL}) \mathrm{ML}$

Level: (low/med) LOW

\% Moisture: not dec.

GC Column: RTX-624 ID: 0.18 (mm)

Soil Extract Volume:

(uL)
Contract: $\mathrm{N} / \mathrm{A}$

SAS NO. : N/A
ARGONNE SAMPLE NO.

MRMWIS-W-23262

Lab Sample ID: 0080410-04

Lab File ID: H001095

Date Received: 04/23/08

Date Analyzed: 04/23/08

Dilution Factor: 1.0

Soil Aliquot Volume:

(uL)

CONCENTRATION UNITS:

CAS NO.

COMPOUND

(ug/L or $\mathrm{ug} / \mathrm{Kg}$ ) UG/L

Q

75-71-8-------Dichlorodifluoromethane

74-87-3------Chloromethane

75-01-4------vinyl Chloride

74-83-9-....-.-Bromomethane

75-00-3-......... Chloroethane

75-69-4------ Trichlorofluoromethane

75-35-4--.-.-1, I-Dichloroethene

76-13-1-...-1, 1, 2-Trichloro-1, $\overline{2,2-t r i f l u}$

67-64-1--.---Acetone

75-15-0--..--Carbon Disulfide

79-20-9-....-. Methyl Acetate

75-09-2-...-.--Methylene Chloride

156-60-5-------trans-1,2-Dichloroethene

1634-04-4-.---Methyl tert-Butyl Ether

75-34-3--n-.-1, 1-Dichloroethane

156-59-2-------cis-1,2-Dichloroethene

78-93-3-.....-2-Butanone

67-66-3-...-.-Chloroform

71-55-6--.---1, 1, 1-Trichloroethane

110-82-7--.---Cyclohexane

56-23-5--..--Carbon Tetrachloride

71-43-2--.----Benzene

107-06-2-----1,2-Dichloroethane

79-01-6-...-. Trichloroethene

108-87-2------Methyl cyclohexane

78-87-5--...-1, 2-Dichloropropane

75-27-4-...---Bromodichloromethane

10061-01-5-----cis-1,3-Dichloropropene

108-10-1------4-Methyl-2-Pentanone

108-88-3--.-.-.Toluene

10061-02-6-----trans-1, 3-Dichloropropene

79-00-5-....-1,1,2-Trichloroethane

127-18-4-..---Tetrachloroethene

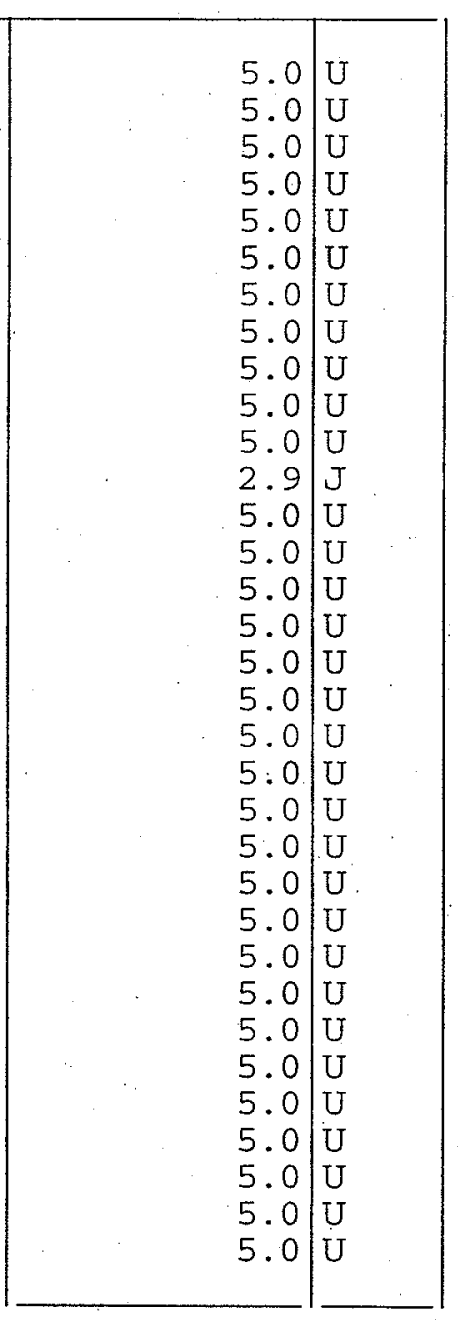


FORM 1

VOLATILE ORGANICS ANALYSIS DATA SHEET

Lab Name: ENVIROSYSTEMS, INC.

Lab Code: ENvSYS Case No.:

Matrix: (soil/water) WATER

Sample wt/vol:

$5.000(\mathrm{~g} / \mathrm{mL}) \cdot \mathrm{ML}$

Level : (low/med). LOW

응 Moisture: not dec.

GC Column: RTX-624 ID: 0.18 (mm)

Soil Extract Volume:

(uis)
Contract: N/A

SAS NO.: N/A
ARGONNE SAMPLE NO.

MRMWIS-W-23262

Lab Sample ID: 0080410-04

Lab File ID: H001095

Date Received: 04/23/08

Date Analyzed: 04/23/08

Dilution Factor: 1.0

Soil Ailquot Volume: (uL) CONCENTRATION UNITS:

CAS NO. COMPOUND $(\mathrm{ug} / \mathrm{L}$ or $\mathrm{ug} / \mathrm{Kg}) \mathrm{UG} / \mathrm{L}$

Q

$5.0 \mathrm{U}$

$5.0 \mathrm{U}$

$5.0 \mathrm{U}$

$5.0 \mathrm{U}$

$5.0 \mathrm{U}$

$5.0 \mathrm{U}$

$5.0 \mathrm{U}$

$5.0 \mathrm{U}$

$5.0 \mathrm{U}$

$5.0 \mathrm{U}$

$5.0 \mathrm{U}$

$5.0 \mathrm{U}$

$5.0 \mathrm{U}$

$5.0 \mathrm{U}$

$5.0 \mathrm{U}$

$10 \mathrm{U}$

$5.0 \mathrm{U}$

$10 \mathrm{U}$

$10 \mathrm{U}$

$10 \mathrm{U}$

$10 \mathrm{U}$ 
FORM 1

VOLATILE ORGANICS ANALYSIS DATA SHEET

Lab Name: ENVIROSYSTEMS, INC.

Lab Code: ENVSYS Case No.:

Matrix: (soil/water) WATER

Sample wt/vol: $\quad 5.000(\mathrm{~g} / \mathrm{mL})$ ML

Level: (low/med). LOW

\% Moisture: not dec.

GC Column: RTX-624 ID: 0.18 (mm)

Soil Extract Volume:

(uL)
Contract: N/A

SAS NO.: N/A
ARGONNE SAMPLE NO.

MRMWIS-W-23263

Lab Sample ID: 0080410-06

Lab File ID: H001097

Date Received: 04/23/08

Date Analyzed: 04/23/08

Dilution Factor: 1.0

Soil Aliquot Volume: (UI)

CONCENTRATION UNITS:
$(\mathrm{ug} / \mathrm{L}$ or $\mathrm{ug} / \mathrm{Kg}$ ) UG/L

Q

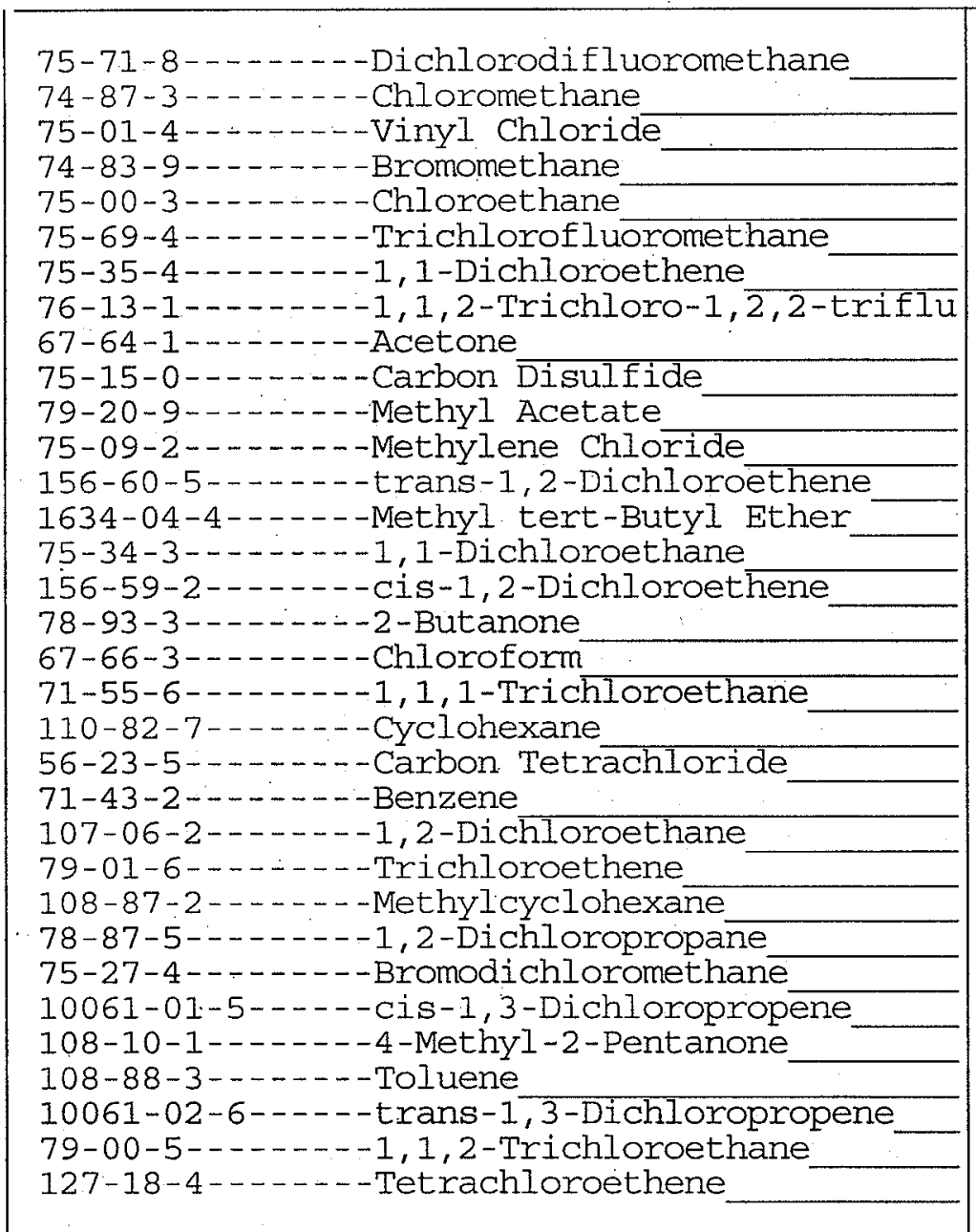

$5.0 \mathrm{U}$

$5.0 \mathrm{U}$

$5.0 \mathrm{U}$

$5.0 \mathrm{U}$

$5.0 \mathrm{U}$

$5.0 \mathrm{U}$

$5.0 \mathrm{U}$

$5.0 \mathrm{U}$

7.2

$5.0 \overline{\mathrm{U}}$

$5.0 \mathrm{U}$

$2.9 \mathrm{~J}$

$5.0 \mathrm{U}$

$5.0 \mathrm{U}$

$5.0 \mathrm{U}$

$5.0 \mathrm{U}$

$5.0 \mathrm{U}$

$5.0 \mathrm{U}$

$5.0 \mathrm{U}$

$5.0 \mathrm{U}$

$5.0 \mathrm{U}$

$5.0 \mathrm{U}$

$5.0 \mathrm{U}$

$5.0 \mathrm{U}$

$5.0 \mathrm{U}$

$5.0 \mathrm{U}$

$5.0 \mathrm{U}$

$5.0 \mathrm{U}$

$5.0 \mathrm{U}$

$1.7 \mathrm{~J}$

$5.0 \mathrm{U}$

$5.0 \mathrm{U}$

$5.0 \mathrm{U}$ 
FORM 1 VOLATILE ORGANICS ANALYSIS DATA SHEET

Lab Name: ENVIROSYSTEMS, INC. Lab Code: ENvSYS Case No.: Matrix: (soil/water) WATER

Sample wt/vol: $\quad 5.000(\mathrm{~g} / \mathrm{mL}) \mathrm{ML}$

Level: (low/med) LOW

\% Moisture: not dec.

GC Column: RTX-624 ID: 0.18 (mm)

Soil Extract Volume:

(uL)
Contract: $\mathrm{N} / \mathrm{A}$

SAS NO.: N/A
ARGONNE SAMPLE NO.

MRMWIS-W-23263

Lab Sample ID: 0080410-06

Lab File ID: H001097

Date Received: 04/23/08

Date Analyzed: 04/23/08

Dilution Factor: 1.0

Soil Aliquot Volume:

(UL)

CONCENTRATION UNITS:

(ug/L or $u g / \mathrm{Kg}$ ) UG/L Q

$5.0 \mathrm{U}$

$5.0 \mathrm{U}$

$5.0 \mathrm{U}$

$5.0 \mathrm{U}$

$5.0 \mathrm{U}$

$5.0 \mathrm{U}$

$5.0 \mathrm{U}$

$5.0 \mathrm{U}$

$5.0 \mathrm{U}$

$5.0 \mathrm{U}$

$5.0 \mathrm{U}$

$5.0 \mathrm{U}$

$5.0 \mathrm{U}$

$5.0 \mathrm{U}$

$5.0 \mathrm{U}$

$10 \mathrm{U}$

$5.0 \mathrm{U}$

$10 \mathrm{U}$

$10 \mathrm{U}$

$10 \mathrm{U}$

$10 \mathrm{U}$ 
FORM 1

VOLATILE ORGANICS ANALYSIS DATA SHEET

Lab Name: ENVIROSYSTEMS, INC.

Lab Code: ENVSYS Case No.:

Matrix: (soil/water) WATER

Sample wt/vol: $\quad 5.000(\mathrm{~g} / \mathrm{mL}) \mathrm{ML}$

Level: (low/med) LOW

\% Moisture: not dec.

GC Column: RTX-624 ID: 0.18 (mm)

Soil Extract Volume:

(UI)
Contract: N/A

SAS NO.: N/A
ARGONNE SAMPLE NO.

MRMWIS-W-23264

Lab Sample ID: 0080410-05

Lab File ID: $\quad$ H001096

Date Received: 04/23/08

Date Analyzed: 04/23/08

Dilution Factor: 1.0

Soil Aliquot Volume:

CONCENTRATION UNITS:

(ug/L or $u g / \mathrm{Kg}$ ) UG/L

Q

\begin{tabular}{|c|c|}
\hline 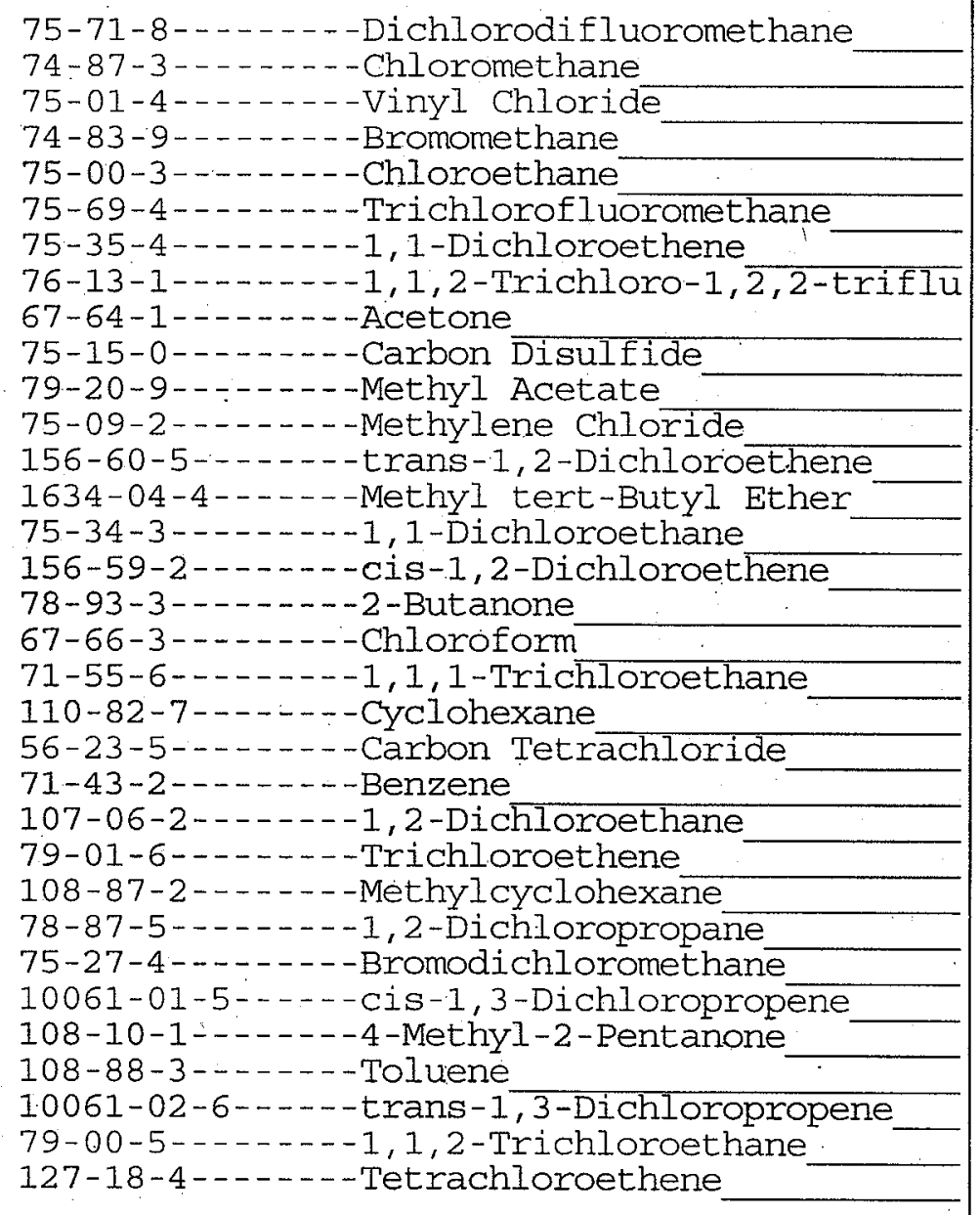 & $\begin{array}{l}5.0 \\
5.0 \\
5.0 \\
5.0 \\
5.0 \\
5.0 \\
5.0 \\
5.0 \\
5.0 \\
5.0 \\
5.0 \\
3.1 \\
5.0 \\
5.0 \\
5.0 \\
5.0 \\
5.0 \\
5.0 \\
5.0 \\
5.0 \\
5.0 \\
5.0 \\
5.0 \\
5.0 \\
5.0 \\
5.0 \\
5.0 \\
5.0 \\
5.0 \\
5.0 \\
5.0 \\
5.0 \\
5.0\end{array}$ \\
\hline
\end{tabular}

FORM I VOA 
FORM 1

VOLATILE ORGANICS ANALYSIS DATA SHEET

Lab Name: ENVIROSYSTEMS, INC.

Lab Code: ENVSYS Case No.:

Matrix: (soil/water) WATER

Sample wt/vol: $\quad 5.000(\mathrm{~g} / \mathrm{mL}) \mathrm{ML}$

Level: (low/med) LOW

\% Moisture: not dec.

GC Column: RTX-624 ID: 0.18 (mm)

Soil Extract Volume: (uL)
Contract: N/A

SAS NO.: N/A
ARGONNE SAMPLE NO.

MRMWIS-W-23264

Lab Sample ID: 0080410-05

Lab File ID: $\mathrm{H} 001096$

Date Received: 04/23/08

Date Analyzed: 04/23/08

Dilution Factor: 1.0

Soil Aliquot Volume: (uL) CONCENTRATION UNITS: CAS NO. COMPOUND $\quad(u g / L$ or $\mathrm{ug} / \mathrm{Kg})$ UG/L

\section{Q}

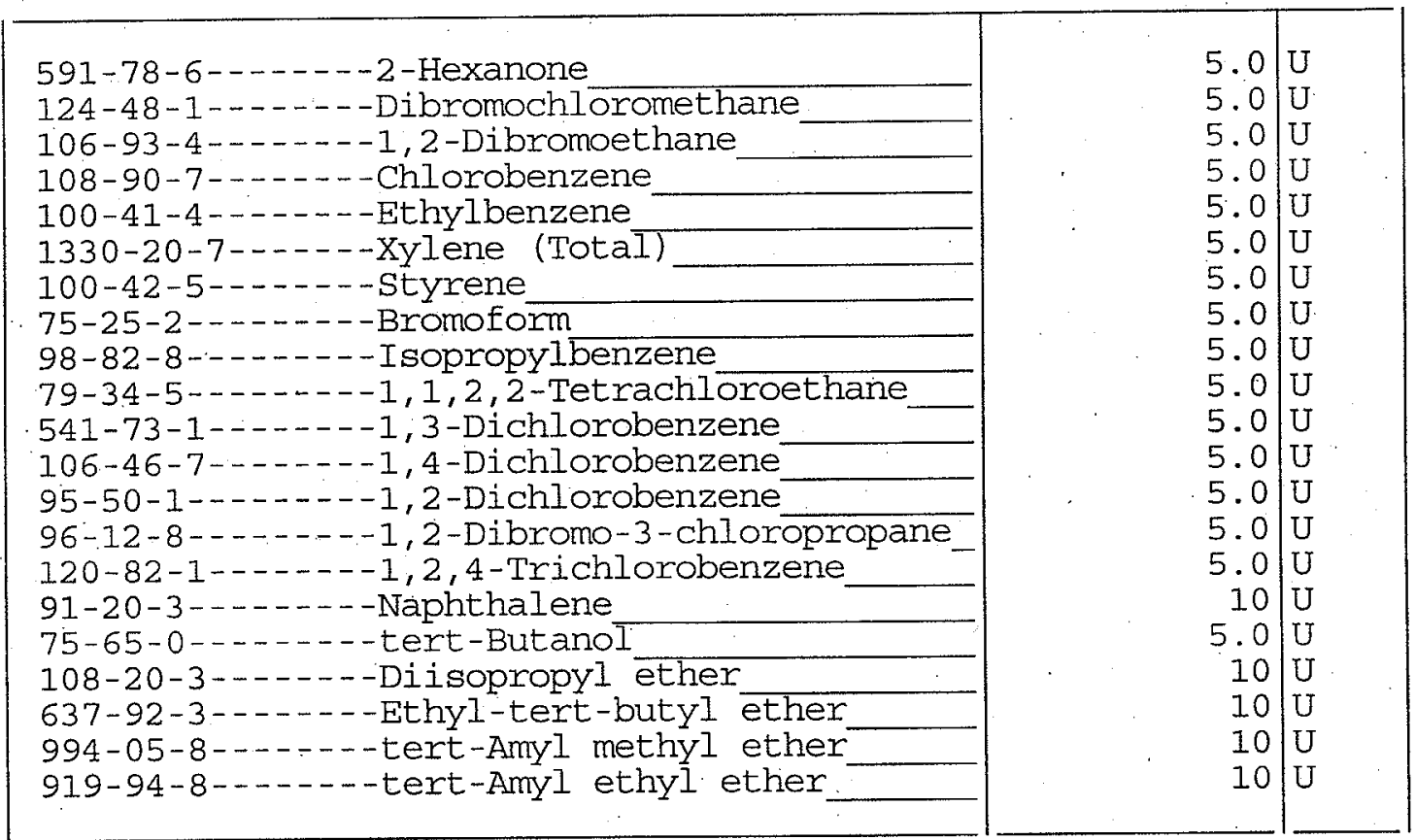


FORM 1

VOLATILE ORGANICS ANALYSIS DATA SHEET

Lab Name: ENVIROSYSTEMS, INC.

Lab Code: ENVSYS Case No.:

Matrix: (soil/water) WATER

Sample wt/vol:

$5.000(\mathrm{~g} / \mathrm{mL}) \mathrm{ML}$

Level : (low/med) LOW

$\div$ Moisture: not dec.

GC Column: RTX-624 ID: 0.18 (mm)

Soil Extract Volume:

(UL)
Contract: N/A

SAS NO.: N/A
ARGONNE SAMPLE NO.

VBLKGK

SDG No.: NA

Lab Sample ID: 0000852-BLK1

Lab File ID: H001090

Date Received:

Date Analyzed: 04/23/08

Dilution Factor: 1.0

Soil Aliquot Volume:

(u亡)

CONCENTRATION UNITS:

CAS NO.

COMPOUND

(ug/L or $\mathrm{ug} / \mathrm{Kg})$. UG/L

Q

\begin{tabular}{|c|c|}
\hline $\begin{array}{l}75-71-8-\cdots \\
74-87-3-1,-1\end{array}$ & $\left.\begin{array}{r|}5.0 \\
5.0 \\
5.0 \\
5.0 \\
5.0 \\
5.0 \\
5.0 \\
5.0 \\
5.0 \\
5.0 \\
5.0 \\
3.0 \\
5.0 \\
5.0 \\
5.0 \\
5.0 \\
5.0 \\
5.0 \\
5.0 \\
5.0 \\
5.0 \\
5.0 \\
5.0 \\
5.0 \\
5.0 \\
5.0 \\
5.0 \\
5.0 \\
5.0 \\
5.0 \\
5.0 \\
5.0 \\
5.0 \\
5.0\end{array}\right\}$ \\
\hline
\end{tabular}


FORM 1

VOLATILE ORGANICS ANALYSIS DATA SHEET

Lab Name: ENVIROSYSTEMS, INC.

Lab Code: ENVSYS Case No.:

Matrix: (soil/water) WATER

Sample wt/vol: $\quad 5.000(\mathrm{~g} / \mathrm{mL})$. ML

Level: (low/med) LOW

\% Moisture: not dec.

GC Column: RTX-624 ID: 0.18 (mm)

Soil Extract Volume:

(uL)
Contract: N/A

SAS NO.: N/A
ARGONNE SAMPLE NO

VBLKGK

Lab Sample ID: 0000852-BLK1

Lab File ID: H001090

Date Received:

Date Analyzed: 04/23/08

Dilution Factor: 1.0

Soil Aliquot Volume:

(UI)

CONCENTRATION UNITS :

(ug/L or $\mathrm{ug} / \mathrm{Kg}$ ) UG/L . Q

591-78-6--.-.--2-Hexanone

124-48-1--.---Dibromochloromethane

106-93-4-..- - 1, 2-Dibromoethane

108-90-7-...-.-Chlorobenzene

100-41-4--.---Ethylbenzene

1330-20-7--.--xylene (Total)

100-42-5--...--styrene

75-25-2-------Bromoform

98-82-8-..-.-.-Isopropyl benzene

79-34-5 .....- 1, 1,2,2-Tetrachloroethane

$541-73-1 \ldots-\cdots-1,3-$ Dichlorobenzene

1.06-46-7--.---1,4-Dichlorobenzene

95-50-1-.....-1, 2-Dichlorobenzene

96-12-8--.----1,2-Dibromo-3-chloropropane

120-82-1-..-- 1, 2, 4-Trichlorobenzene

91-20-3-....-Naphthalene

75-65-0--.----tert-Butanol

108-20-3--.--Diisopropyl ether

637-92-3-...--Ethyl-tert-butyl ether

994-05-8-..---tert-Amyl methyl ether

919-94-8------tert-Amyl ethyl ether
$5.0 \mathrm{U}$

$5.0 \mathrm{U}$

$5.0 \mathrm{U}$

$5.0 \mathrm{U}$

$5.0 \mathrm{U}$

$5.0 \mathrm{U}$

$5.0 \mathrm{U}$

$5.0 \mathrm{U}$

$5.0 \mathrm{U}$

$5.0 \mathrm{U}$

$5.0 \mathrm{U}$

$5.0 \mathrm{U}$

$5.0 \mathrm{U}$

$5.0 \mathrm{U}$

$5.0 \mathrm{U}$

$10 \mathrm{U}$

$5.0 \mathrm{U}$

$10 \mathrm{U}$

$10 \mathrm{U}$

$10 \mathrm{U}$

$10 \mathrm{U}$ 


\section{ENVIROSYSTEMS, INC.}

9200 Rumsey Road - Suite B102 - Columbia, Maryland 21045-1934

Phone (410) 964-0330 - Fax (410) 740-9306

Email: info@envsystems.com - Webpage: www.envsystems.com/envsys

Date: June-11-2008

Name: - Jorge S. Alvarado Ph.D

Company: Argonne National Laboratory

Address: Enviromental Research Division

Applied Geosciences and Enviromental

Management Section

9700 South Cass Avenue

BIdg: 203, Room \# A137

Lemont, IL 60439

RE: Repor\# 0080416

Dear Jorge:

Enclosed are the results of analyses for samples received by the laboratory on May 09 , 2008 . If you have any questions concerning this report, please feel free to contact me.

Please do not hesitate to call if you have any questions, comments or require additional information

Sincerely:

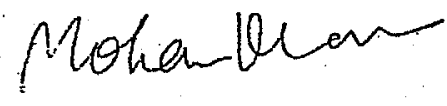

Mohan Khare. Ph.D

Presideni/ CEO

Report \# 080190 
1. Narrative 


\section{Narrative}

This analytical data package contains the volatile organic analysis by USEPA SW-846 Method 8260B and CLP protocols for samples received April 25, 2008

The chain of custody document for this report is in section 2, the analytical data summary, sample data, and standard data is in section 3 . 
2. Traffic Repoits/ Chain of Custody Records 


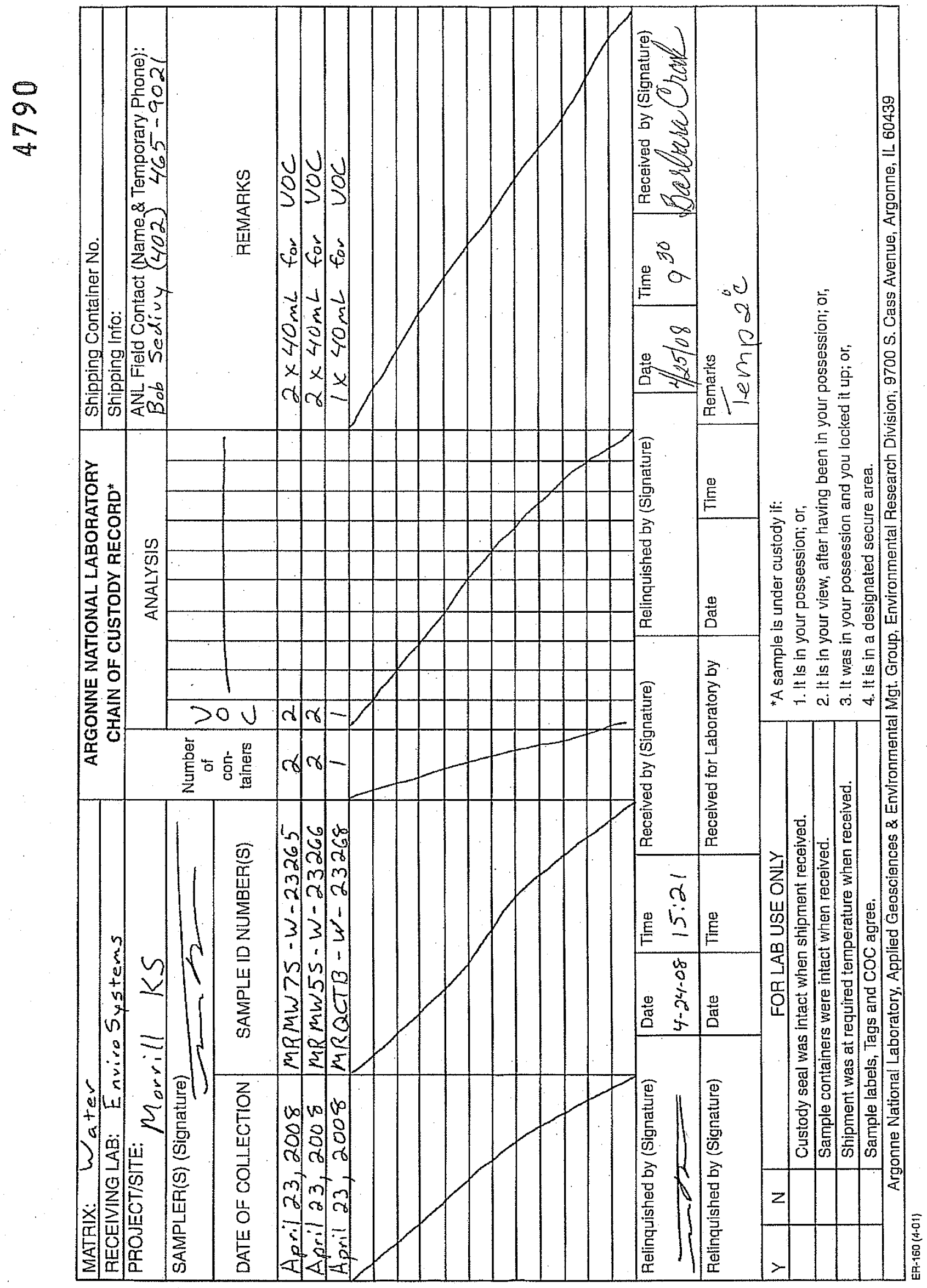


3. VOA Data 
FORM 1

VOIATILE ORGANICS ANALYSIS DATA SHEET

Lab Name: ENVIROSYSTEMS, INC.

Lab Code: ENvSYs Case No.:

Matrix: (soil/water) WATER

Sample wt/vol: $\quad 5.000(\mathrm{~g} / \mathrm{mL}) \mathrm{ML}$

Level: (low/med) LOW

을 Moisture: not dec.

GC Column: RTX-624 ID: 0.18 (mm)

Soil Extract Volume:

(uL.)
Contract : N/A

SAS NO.: N/A
ARGONNE SAMPLE NO.

MRMW75-W-23265

SDG NO. : NA

Lab Sample ID: 0080416-04

Lab File ID: H001290

Date Received: 04/25/08

Date Analyzed: 05/06/08

Dilution Factor: 1.0

Soil Aliquot Volume:

(UI)

CONCENTRATTON UNITS :

CAS NO. COMPOUND (ug/I or ug/Kg) UG/L

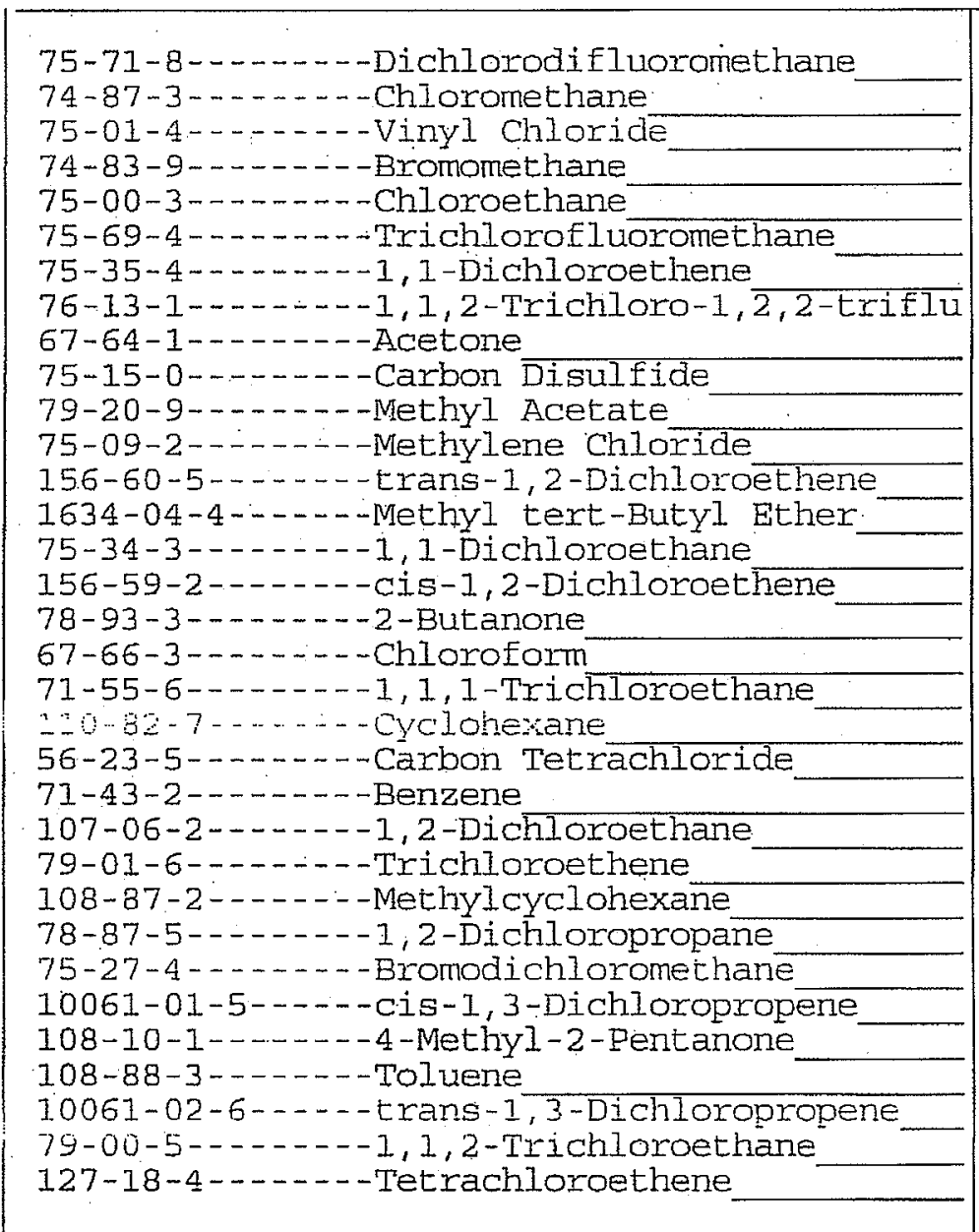

$5.0 \mathrm{U}$

$5.0 \mathrm{U}$

$5.0 \mathrm{U}$

$5.0 \mathrm{U}$

$5.0 \mathrm{U}$

$5.0 \mathrm{U}$

$5.0 \mathrm{U}$

$5.0 \mathrm{U}$

$5.0 \mathrm{U}$

5:0 U

$5.0 \mathrm{U}$

$4.5 \mathrm{~J}$

$5.0 \mathrm{U}$

$5.0 \mathrm{U}$

$5.0 \mathrm{U}$

$5.0 \mathrm{U}$

$5.0 \mathrm{U}$

$5.0 \mathrm{U}$

$5.0 \mathrm{U}$

$5.0 \mathrm{U}$

7.6

$5.0 \mathrm{U}$

$5.0 \mathrm{U}$

$5.0 \mathrm{U}$

$5.0 \mathrm{U}$

$5.0 \mathrm{U}$

$5.0 \mathrm{U}$

$5.0 \mathrm{U}$

I.2 J

$5.0 \mathrm{U}$

$5.0 \mathrm{U}$

$5.0 \mathrm{U}$

$5.0 \mathrm{U}$ 
FORM 1

VOLATILE ORGANICS ANALYSIS DATA SHEET

Lab Name: ENVIROSYSTEMS, INC.

Contract: $\mathrm{N} / \mathrm{A}$

Lab Code: ENVSYS Case No.:

Matrix: (soil/water) WATER

Sample wt/vol:

$5.000(\mathrm{~g} / \mathrm{mL}) \mathrm{ML}$

Level: (low/med) LOW

: Moisture: not dec.

GC Column: RTX-624

ID: $0.18(\mathrm{~mm})$

SAS NO.: N/A
ARGONNE SAMPLE NO.

MRMW75-W-23265

Soil Extract Volume: (uT)

Lab Sample ID: 0080416-04

Lab File ID: Ho01290

Date Received: 04/25/08

Date Analyzed: 05/06/08

Dilution Factor: 1.0

Soil Aliquot Volume: (uU)

CAS NO.

COMPOUND

CONCENTRATION UNITS:

(ug/L or $\mathrm{ug} / \mathrm{Kg}$ ) UG/L

Q

$5.0 \mathrm{U}$

$5.0 \mathrm{U}$

$5.0 \mathrm{U}$

$5.0 \mathrm{U}$

$5.0 \mathrm{U}$

$5.0 \mathrm{U}$

$5.0 \mathrm{U}$

$5.0 \mathrm{U}$

$5.0 \mathrm{U}$

$5.0 \mathrm{U}$

$5.0 \mathrm{U}$

$5.0 \mathrm{U}$

$5.0 \mathrm{U}$

$5.0 . \mathrm{U}$

$5.0 \mathrm{U}$

$10 \mathrm{U}$

$5.0 \mathrm{U}$

$10 \mathrm{U}$

100

$10 \mathrm{U}$

$10 \mathrm{U}$

994-05-8--.--tert-Anyl methyl ether 919-94-8-.....tert-Amyl ethyl ether 
FORM 1

VOLATILE ORGANICS ANATYSIS DATA SHEET
ARGONNE SAMPIE NO.

MRMN55-W-23266
Lab Name: ENVIROSYSTEMS, INC.

Lab Code: ENVSYS Case No.:

Matrix: (soil/water) WATER

Sample wt/vol: $\quad 5.000(\mathrm{~g} / \mathrm{mL}) \mathrm{ML}$

Level: (low/med) LOW

․ Moisture: not dec.

GC Column: RTX-624 ID: 0.18 (mm)

Soil Extract Volume:

(UT)
Contract: $N / A$

SAS NO.: N/A. SDG NO.: NA

Lab Sample ID: 0080416-05

Lab File ID: H001291

Date Received: 04/25/08

Date Analyzed: 05/06/08

Dilution Factor: 1.0

Soil Aliquot Volume: (UI)

(ug/L or $\mathrm{ug} / \mathrm{kg}$ ) UG/L Q

$5.0 \mathrm{U}$

$5.0 \mathrm{U}$

$5.0 \mathrm{U}$

$5.0 \mathrm{U}$

$5.0 \mathrm{U}$

$5.0 \mathrm{U}$

$5.0 \mathrm{U}$

$5.0 \mathrm{U}$

9.5

$5.0 \overline{\mathrm{U}}$

$5.0 \mathrm{U}$

6.2 .

$5.0 . \overline{\mathrm{U}}$

$5.0 \mathrm{U}$

$5.0 \mathrm{U}$

$5.0 \mathrm{U}$

$5.0 \mathrm{U}$

$5.0 \mathrm{U}$

$5.0 \mathrm{U}$

$5.0 \mathrm{U}$

$5.0 \mathrm{U}$

$5.0 . \mathrm{U}$

$5.01 \mathrm{U}$

$5.0 \mathrm{U}$

$5.0 \mathrm{U}$

$5.0 \mathrm{U}$

$5.0 \mathrm{U}$

$5.0 \mathrm{U}$

1.2 J

$5.0 \mathrm{U}$

$5.0 \mathrm{U}$

$5.0 \mathrm{U}$

$5.0 \mathrm{U}$

FORM I VOA 
FORM 1

VOLATILE ORGANICS ANALYSIS DATA SHEET

Lab Name: ENVIROSYSTEMS, INC.

Lab Code: ENVSYS Case No.:

Matrix: (soil/water) WATER

Sample wt/vol: $\quad 5.000(\mathrm{~g} / \mathrm{mL}) \mathrm{ML}$

Level: (low/med) Low

\% Moisture: not dec.

GC Column: RTX-624 ID: 0.18 (mm)

Soil Extract Volume:

\{uบ)
Contract: $\mathrm{N} / \mathrm{A}$

SAS NO.: N/A
ARGONNE SAMPLE NO.

MRMW55-W-23266

Lab Sample ID: 0080416-05

Lab File ID: Ho01291

Date Received: 04/25/08

Date Analyzed: 05/06/08

Dilution Factor: 1.0

Soil Aliquot Volume: (UI)

CONCENTRATION UNITS:

(ug/I or $\mathrm{ug} / \mathrm{Kg}$ ) UG/L

Q

$591-78-6-----2-H e x a n o n e$

124-48-1...... Dibromochloromethane

106-93-4-..--1,2-Dibromoethane

108-90-7--..--Chlorobenzene

100-41-4-....-Ethylbenzene

1330-20-7-..-XXlene (TotaI)

100-42-5------Styrene

75-25-2-....-Bromoform

98-82-8-------Isopropyl benzene

$79-34-5-\ldots-1,1,2,2-$ Tetrachloroethane

541-73-1-----1,3-Dichlorobenzene

106-46-7-----1,4-Dichlorobenzene

95-50-1 -..-- - 1, 2-Dichlorobenzene

96-12-8-..---1,2-Dibromo-3-chloropropane

120-82-1-....-1,2,4-Trichlorobenzene

91-20-3-...-.-Naphthalene

75-65-0-....--tert-Butanol

108-20-3-....-Di Dopropy l ether

637-92-3-....-Ethyl-tert-butyl ether

994-05-8-...-tert-Amyl methyl ether

919-94-8------tert-Amyl ethyl ether

$5.0 \mathrm{U}$

$5.0 \mathrm{U}$

$5.0 \mathrm{U}$

$5.0 \mathrm{U}$

$5.0 \mathrm{U}$

$5.0 \mathrm{U}$

$5.0 \mathrm{U}$

$5.0 \mathrm{U}$

$5.0 \mathrm{U}$

$5.0 \mathrm{U}$

$5.0 \mathrm{U}$

$5.0 \mathrm{U}$

$5.0 \mathrm{U}$

$5.0 \mathrm{U}$

$5.0 \mathrm{U}$

$10 \mathrm{U}$

$5.0 \mathrm{U}$

$10 \mathrm{U}$

$10 \mathrm{U}$

$10 \mathrm{U}$

$10 \mathrm{U}$

FORM I VOA 
FORM 1

VOLATILE ORGANICS ANALYSIS DATA SHEET

Lab Name: ENVIROSYSTEMS, INC.

Lab Code: ENVSYS Case No.:

Matrix: (soil/water) WATER

Sample wt/vol: $\quad 5.000(\mathrm{~g} / \mathrm{mL}) \mathrm{ML}$

Level: (low/med) LOW

\% Moisture: not dec.

GC Column: RTK-624 ID: 0.18 (mm)

Soil Extract Volume:

(UE)
Contract: $\mathrm{N} / \mathrm{A}$

SAS NO.: N/A
ARGONNE SAMPLE NO.

MRQCTB-W-23268
SDG NO. : NA

Lab Sample ID: 0080416-06

Lab File ID: Ho0I292

Date Received: 04/25/08

Date Analyzed: 05/06/08

Dilution Factor: 1:0

Soil Aliquat Volume:

(ut)

CAS NO. COMPOUND (ug/L or ug/Kg) UG/L

$Q$

$5.0 \mathrm{U}$

$5.0 \mathrm{U}$

$5.0 \mathrm{U}$

$5.0 \mathrm{U}$

$5.0 \mathrm{U}$

$5.0 \mathrm{U}$

$5.0 \mathrm{U}$

$5.0 \mathrm{U}$

12

$5.0 \overline{\mathrm{U}}$

$5.0 \mathrm{U}$

9.1

$5.0 \overline{\mathrm{U}}$

$5.0 \mathrm{U}$

$5.0 \mathrm{U}$

$5.0 \mathrm{U}$

$5.0 \mathrm{U}$

5.0 U

$5.0 \mathrm{U}$

5. 0 U

5.0 U

$5.0 \mathrm{U}$

$5.0 \mathrm{U}$

$5.0 \mathrm{U}$

$5.0 \mathrm{U}$

$5.0 \mathrm{U}$

$5.0 \mathrm{U}$

$5.0 \mathrm{U}$

$5.0 \mathrm{U}$

$5.0 \mathrm{U}$

5.0 U

$5.0 \mathrm{U}$

$2.5 \mathrm{~J}$ 
FORM I

VOLATILE ORGANICS ANALYSIS DATA SHEET
ARGONNE SAMPLE NO.

MRQCTB-W-23268
Lab Name: ENVIROSYSTEMS, INC.

Lab Code: ENVSYS Case No.:

Matrix: (soil/water) WATER

Sample wt/vol:

$5.000(\mathrm{~g} / \mathrm{mL}) \mathrm{NL}$

Level: (low/med) LOW

\% Moisture: not dec.

GC Column: RTX-624 ID: 0.18 (mm)

Soil Extract Volume:
Contract: $N / A$

SAS NO.: N/A

Lab Sample ID: 0080416-06

Lab File ID: H001292

Date Received: 04/25/08

Date Analyzed: 05/06/08

Dilution Factor: 1.0

Soil Aliquot Volume: (uU)

CONCENTRATION UNITS:

(ug/L or ug/kg) UG/L Q

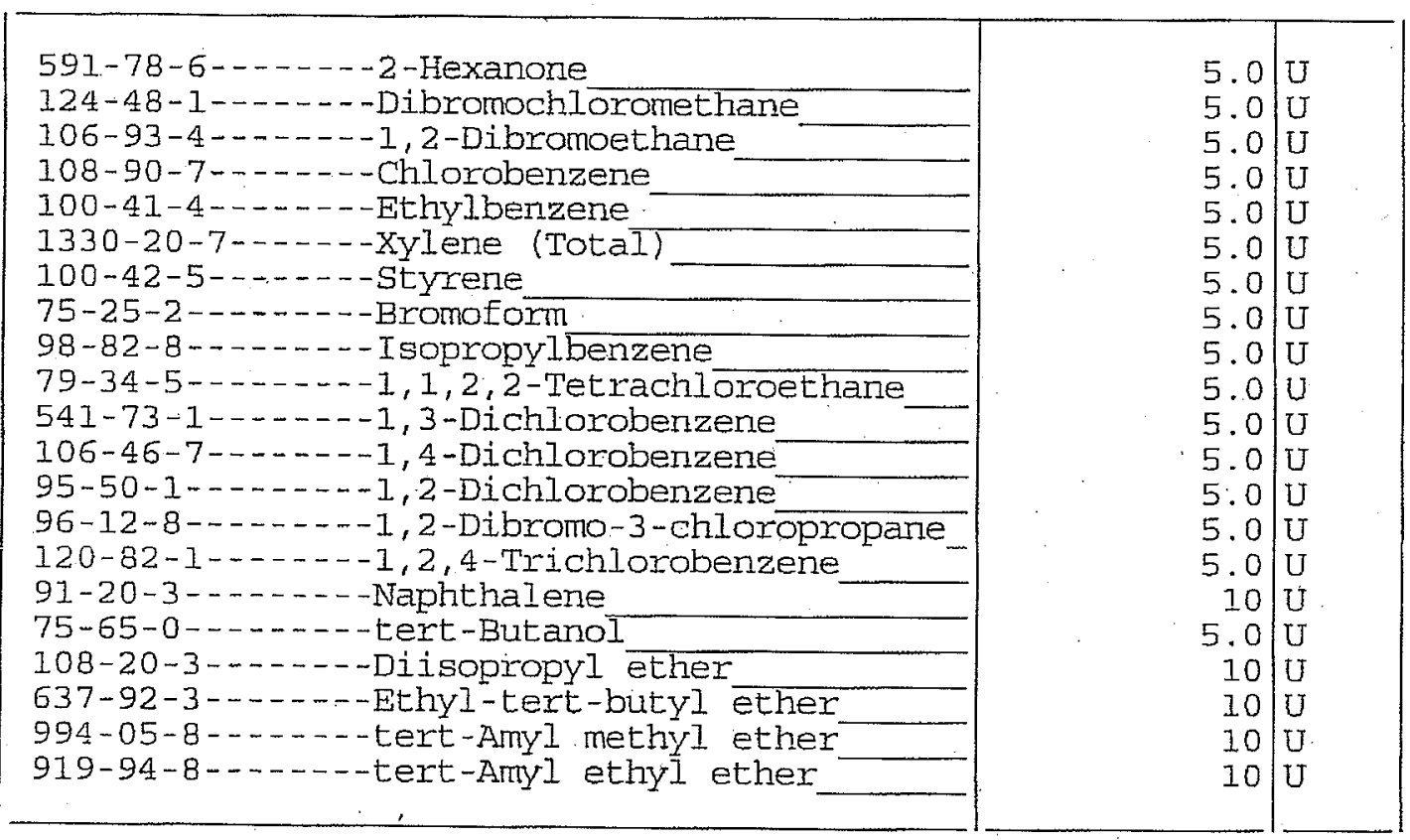


FORM 1

VOLATILE ORGANICS ANALYSIS DATA SHEET

Lab Name: ENVIROSYSTEMS, INC.

Lab Code: ENvSYS Case No.:

Natrix: (soil/water) WATER

Sample wt/vol:

$5.000(\mathrm{~g} / \mathrm{mL}) \mathrm{ML}$

Level: (low/med) LOW

o Moisture: not dec.

GC Column: RTX-624 ID: 0.18 (mm)

Soil Extract Volume:

(UI)
Contract: $\mathrm{N} / \mathrm{A}$

SAS NO.: N/A
ARGONNE SAMPLE NO.

VBLKHP

Lab Sample ID: 0000873-BLKI.

Lab File ID: Ho01286

Date Received:

Date Analyzed: 05/05/08

Dilution Factor: 1.0

Soil Aliquot Volume:

(uL)

CONCENTRATION UNITS :

(ug/L or ug/ $\mathrm{kg}$ ) UG/L

2

75-71-8--.--Dichlorodifluoromethane

74-87-3-...--Chloromethane

75-01-4-..---vinyl Chloride

74-83-9-...-- Bromomethane

75-00-3-------Chloroethane

75-69-4------Trichlorofluoromethane

75-35-4-....-1,1-Dichloroethene

$76-13-1-\ldots-\cdots-1,1,2-T r i c h l o r o-1, \overline{2,2-t r i f l u}$

$67-64-1 \ldots \ldots-\ldots$ Acetone

75-15-0.....-.-Carbon Disulfide

79-20-9--.---Methyl Acetate

75-09-2-.....-. Methylene Chloride

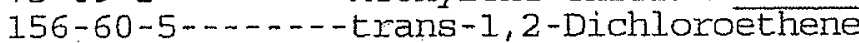

1634-04-4-----Methyl tert-Butyl Ether

75-34-3-...---1, 1-Dichloroethane

156-59-2-...--cis-1,2-Dichloroethene

78-93-3-------2-Butanone

67-66-3--.---Chloroform

71-55-6------ 1, 1, 1-Trichloroethane

110-82-7--..-cyclohexane

56-23-5-....-Carbor Tetrachloride

$71-43-2-\ldots-\cdots-B e n z e n e$

107-06-2-...-1,2-Dichloroethane

79-01-6--...-. Trichloroethene

108-87-2-...--Nethylcyclohexane

78-87-5-...- 1, 2-Dichloropropane

75-27-4---1---Bromodichloromethane

10061-01-5-----cis-1,3-Dichloropropene

108-10-1-...--4-Methyl-2-Pentanone

108-88-3......-Toluene

10061-02-6-.....trans-1,3-Dichloropropene

79-00-5-....-1, 1, 2-Trichloroethane

127-18-4---n-Tetrachloroethene
$5.0 \mathrm{U}$

$5.0 \mathrm{U}$

$5.0 \mathrm{U}$

$5.0 \mathrm{U}$

$5.0 \mathrm{U}$

$5.0 \mathrm{U}$

$5.0 \mathrm{U}$

$5.0 \mathrm{U}$

$5.0 \mathrm{U}$

$5.0 \mathrm{U}$

$5.0 \mathrm{U}$

$3.4 \mathrm{~J}$

$5.0 \mathrm{U}$

$5.0 \mathrm{U}$

$5.0 \mathrm{U}$

$5.0 \mathrm{U}$

$5.0 \mathrm{U}$

$5.0 \mathrm{U}$

$5.0 \mathrm{U}$

$5.0 \mathrm{U}$

$5.0 \mathrm{U}$

$5.0 \mathrm{U}$

$5.0 \mathrm{U}$

$5.0 \mathrm{U}$

$5.0 \mathrm{U}$

$5.0 \mathrm{U}$

$5.0 \mathrm{U}$

$5.0 \mathrm{U}$

$5.0 \mathrm{U}$

$5.0 \mathrm{U}$

$5.0 \mathrm{U}$

$5.0 \mathrm{U}$

5.00 
FORM 1

VOIATILE ORGANICS ANALYSIS DATA SHEET

Lab Name: ENVIROSYSTEMS, INC.

Lab Code: ENVSYS Case No.:

Matrix: (soil/watex) WATER

Sample wt/vol:

$5.000(\mathrm{~g} / \mathrm{mL}) \mathrm{ML}$

Level : (low/med) LOW

\% Moisture: not dec.

GC Column: RTX-624 ID: 0.18 (mm)

Soil Extract volume:

(uL)
Contract: $\mathrm{N} / \mathrm{A}$

SAS NO.: N/A
ARGONNE SAMPLE NO.

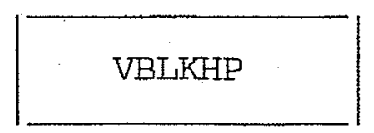

SDG NO.: NA

Lab Sample ID: 0000873-BLKI

Lab File ID: H00I286

Date Received:

Date Analyzed: 05/05/08

Dilution Factor: 1.0

Soil Aliquot Volume:

(uI)

CONCENTRATION UNITS:

CAS NO.

COMPOUND

(ug/L or $\mathrm{ug} / \mathrm{Kg}$ ) UG/L

Q

591-78-6------2-Hexanone

124-48-1-...-.-Dibromochloromethane

106-93-4-------1, 2-Dibromoethane

108-90-7--.-.--Chlorobenzene

100-4I-4-...--.-Ethylbenzene.

1330-20-7-..--Xylene (Total)

100-42-5--.----Styrene

75-25-2-..----Bromoform

98-82-8-...-.-Isopropyl benzene

79-34-5-.....-1, 1, 2, 2-Tetrachloroethane

$541-73-1 \ldots \ldots-\ldots 1,3-D i c h l o r o b e n z e n e$

106-46-7-...--1,4-Dichlorobenzene

95-50-1_....-1, 2-Dichlorobenzene

96-12-8--...-1, 2-Dibromo-3-chloropropane

120-82-1------1,2,4-Trichlorobenzene

91-20-3-..---Naphthalene

75-65-0-.-.-.-tert-Butanol

108-20-3-...-. Di isopropyi ether

637-92-3-..---Ethyl-tert-butyl ether

994-05-8-.---tert-Anyl methyl ether

919-94-8-...--tert-Amyl ethyl ether

\begin{tabular}{ll|l}
5.0 & $\mathrm{U}$ \\
5.0 & $\mathrm{U}$ \\
5.0 & $\mathrm{U}$ \\
5.0 & $\mathrm{U}$ \\
5.0 & $\mathrm{U}$ \\
5.0 & $\mathrm{U}$ \\
5.0 & $\mathrm{U}$ \\
5.0 & $\mathrm{U}$ \\
5.0 & $\mathrm{U}$ \\
5.0 & $\mathrm{U}$ \\
5.0 & $\mathrm{U}$ \\
5.0 & $\mathrm{U}$ \\
5.0 & $\mathrm{U}$ \\
5.0 & $\mathrm{U}$ \\
5.0 & $\mathrm{U}$ \\
10 & $\mathrm{U}$ \\
5.0 & $\mathrm{U}$ \\
10 & $\mathrm{U}$ \\
10 & $\mathrm{U}$ \\
10 & $\mathrm{U}$ \\
10 & $\mathrm{U}$ \\
\hline &
\end{tabular}

FORM I VOA. 
FORM I

VOLATILE ORGANICS ANALYSIS DATA SHEET

Lab Name: ENVIROSYSTEMS, INC.

Lab Code: ENVSYS Case No.:

Matrix: (soil/water) WATER

Sample wt/vol: $\quad 5.000(\mathrm{~g} / \mathrm{mL}) \mathrm{ML}$

Level: (low/med) Low

\% Moisture: not dec.

GC Column: RTX-624 ID: $0.18 \mathrm{(mm})$

Soil Extract Volume:

(uL)
Contract: $N / A$

SAS NO.: N/A
ARGONNE SAMPLE NO.

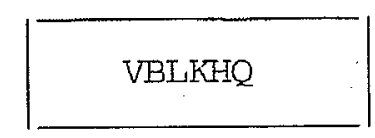

SDG No.: NA

Iab Sample ID: 0000874-BLKI

Lab File ID: F001305

Date Received:

Date Analyzed: 05/06/08

Dilution Factor: 1.0

Soil Aliquot Volume: (uL)

CONCENTRATION UNITS:

CAS NO.

COMPOUND

(ug/L or ug/Kg) UG/L

Q

$5.0 \mathrm{U}$

$5.0 \mathrm{U}$

$5.0 \mathrm{U}$

$5.0 \mathrm{U}$

5.0. U

$5.0 \mathrm{U}$

$5.0 \mathrm{U}$

$5.0 \mathrm{U}$

$5: 0 \mathrm{U}$

$5.0 \mathrm{U}$

$5.0 \mathrm{U}$

$3.9 \mathrm{~J}$

$5.0 \mathrm{U}$

$5.0 \mathrm{U}$

$5.0 \mathrm{U}$

$5.0 \mathrm{U}$

$5.0 \mathrm{U}$

$5.0 \mathrm{U}$

$5.0 \mathrm{U}$

$5.0 \mathrm{U}$

$5.0 \mathrm{U}$

$5.0 \mathrm{U}$

$5.0 \mathrm{U}$

$5.0 \mathrm{U}$

$5.0 \mathrm{U}$

$5.0 \mathrm{U}$

$5.0 \mathrm{U}$

$5.0 \mathrm{U}$

$5.0 \mathrm{U}$

$5.0 \mathrm{U}$

$5.0 \mathrm{U}$

$5.0 \mathrm{U}$

$5.0 \mathrm{U}$ 
FORM 1

VOLATILE ORGANICS ANALYSIS DATA SHEET

Lab Name: ENVIROSYSTEMS, INC.

Lab Code: ENVSYS Case No.:

Matrix: (soil/water) WATER

Sample wt/vol: $\quad 5.000(\mathrm{~g} / \mathrm{mL}) \mathrm{ML}$

Level: (low/med) LOW

$\%$ Moisture: not dec.

GC Column: RTX-624 ID: 0.18 (mm)

Soil Extract Volume:

(UL)
Contract: $N / A$

SAS NO.: N/A
ARGONNE SAMPLE NO.

VBIKKHQ

SDG No.: NA
Lab Sample ID: 0000874-BLKI

Lab File ID: H001305

Date Received:

Date Analyzed: 05/06/08

Dilution Factor: 1.0

Soil Aliquat Volume: (uI)

CAS NO.

COMPOUND

CONCENTRATION UNITS:

(ug/I or $\mathrm{ug} / \mathrm{Kg}$ ) UG/L

Q

$591-78-6-\cdots-\cdots-2-$ Hexanone

124-48-1 -....-Dibromochloromethane

106-93-4--..--1,2-Dibromoethane

108-90-7-....-Chlorobenzene

100-41-4-....--Ethylbenzene

1330-20-7--.--Xylene (Total)

100-42-5--...--styrene

$75-25-2 \ldots \ldots-\ldots$ - Bromoform

98-82-8-..-- - Isopropyl benzene

79-34-5-......-1, 1, 2, 2-Tetrachloroethane

541-73-1_.....1, 3-Dichlorobenzene

106-46-7-...--1, 4-Dichlorobenzene

95-50-1-........ 2, 2-Dichlorobenzene

96-12-8-.....-1, 2-Dibromo-3-chloropropane

120-82-1--.--1, 2, 4-Trichlorobenzene

91-20-3-...-.-Naphthalene

75-65-0--..--tert-Butanol

108-20-3-...--Disopropyl ether

637-92-3-...--Ethyl-tert-butyl ether

994-05-8-...--tert-Antyl methyl ether

919-94-8-....-tert-Amyl ethyl ether
$5.0 \mathrm{U}$

$5.0 \mathrm{U}$

$5.0 \mathrm{U}$

$5.01 \mathrm{U}$

$5.0 . \mathrm{U}$

$5.0 \mathrm{U}$

$5.0 \mathrm{U}$

$5.0 \mathrm{U}$

$5.0 \mathrm{U}$

$5.0 \mathrm{U}$

$5.0 \mathrm{U}$

$5.0 \mathrm{U}$

$5.0 \mathrm{U}$

$5.0 \mathrm{U}$

$5.0 \mathrm{U}$

$10 \mathrm{U}$

$5.0 \mathrm{U}$

$10 \mathrm{U}$

$10 \mathrm{U}$

$10 \mathrm{U}$

$10 \mathrm{U}$ 
Argonne

Environmental Science Division

Argonne National Laboratory

9700 South Cass Avenue, Bldg. 203

Argonne, IL 60439-4843

www.anl.gov

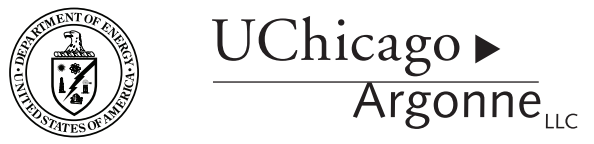

A U.S. Department of Energy laboratory

managed by UChicago Argonne, LLC 